\title{
Álgebras de Krichever-Novikov superelípticas
}

\author{
Felipe Albino dos Santos \\ TESE APRESENTADA AO \\ INSTITUTO DE MATEMÁTICA E ESTATÍSTICA DA \\ UNIVERSIDADE DE SÃo PAULO \\ PARA OBTENÇÃO DO TÍTULO DE \\ DOUTOR EM CIÊNCIAS.
}

Programa: Matemática.

Orientador: Prof. Dr. Vyacheslav Futorny.

Durante o desenvolvimento deste trabalho o autor recebeu auxílio financeiro do CNPq. 


\section{Álgebras de Krichever-Novikov superelípticas}

Esta versão da tese contém as correções e alterações sugeridas pela Comissão Julgadora durante a defesa da versão original do trabalho, realizada em 21/05/2021. Uma cópia da versão original está disponível no Instituto de Matemática e Estatística da Universidade de São Paulo.

Comissão Julgadora:

- Prof. Dr. Vyacheslav Futorny (orientador) - IME-USP

- Prof. Dr. João Fernando Schwarz - IME-USP

- Prof. Dr. Luis Enrique Ramirez - UFABC

- Prof. Dr. Viktor Bekkert - UFMG

- Prof. Dr. Mikhail Neklyudov - UFAM 


\section{Resumo}

SANTOS, Felipe Albino dos. Álgebras de Krichever-Novikov superelípticas. 2021. Tese de Doutorado em Matemática - Instituto de Matemática e Estatística, Universidade de São Paulo, São Paulo, 2021.

Este trabalho apresenta um estudo das álgebras de Krichever-Novikov superelípticas. Seja $p(t) \in \mathbb{C}[t]$ um polinômio com raízes distintas e $\mathfrak{g}$ uma álgebra de Lie simples de dimensão finita sobre os complexos. É apresentada uma descrição em termos de geradores e relações da extensão central universal para a álgebra de Lie afim superelíptica $\mathfrak{g} \otimes R$ em que $R=\mathbb{C}\left[t, t^{-1}, u\right]$ em que $u^{m}=p(t)(m \in \mathbb{N})$. Dos geradores e relações apresentados emergem famílias de polinômios. É apresentada uma análise destas famílias e obtém-se desta análise uma família de polinômios que satisfazem determinada equação diferencial de ordem 4 . Mostra-se que tal família é de polinômios ortogonais não-clássicos. Além disso, neste trabalho é definida a álgebra de Heisenberg hiperelíptica como a subálgebra de Heisenberg da álgebra hiperelíptica laço de Krichever-Novikov. É estabelecido um critério de irredutibilidade explícito para módulos $\varphi$-Verma para estas álgebras. Cada capítulo descreve de forma concisa eventos e personagens da História da Matemática relacionada aos tópicos apresentados.

Palavras-chave: Álgebras de Krichever-Novikov; álgebras superelípticas; álgebras multiponto; álgebras hiperelípticas; polinômios ortogonais; módulos $\varphi$-Verma. 



\section{Abstract}

SANTOS, Felipe Albino dos. Krichever-Novikov superelliptic algebras. 2021. Tese (Doutorado em Matemática) - Instituto de Matemática e Estatística, Universidade de São Paulo, São Paulo, 2021.

The present work addresses the Krichever-Novikov superelliptic algebras. Let $p(t) \in \mathbb{C}[t]$ be a polynomial with distinct roots and $\mathfrak{g}$ a simple Lie algebra over the complex numbers. The universal central extension of the infinite dimensional superelliptic affine Lie algebra $\mathfrak{g} \otimes R$ in which $R=\mathbb{C}\left[t, t^{-1}, u\right]$ where $u^{m}=p(t)$ $(m \in \mathbb{N})$ is described in terms of generators and relations. This description leads to special families of polynomials. We show that these polynomials satisfy certain fourth order differential equations and conclude that they are orthogonal and nonclassical polynomials. Furthermore, we define the hyperelliptic Heisenberg algebra as the Heisenberg subalgebra of a hypereliptic loop Krichever-Novikov algebra and we stabilish a explicit irreducibility criteria for $\varphi$-Verma modules for these algebras. Each chapter provides a short description of events and characters from the History of Mathematics related to the presented topics.

Palavras-chave: Krichever-Novikov algebras; superelliptic algebras; multi-point algebras; orthogonal polynomials; hyperelliptic algebras; $\varphi$-Verma modules. 



\section{Agradecimentos}

Durante os meus últimos anos de estudo, tive a grande oportunidade de contar com pessoas que cruzaram rapidamente o meu caminho de forma passageira, com pessoas que entraram definitivamente na minha vida pessoal e profissional e com pessoas que sempre estiveram e sempre estarão comigo. Todas essas pessoas contribuíram de forma fundamental e única neste trabalho e no meu crescimento pessoal.

Em primeiro lugar, agradeço à minha família pelo apoio incondicional. Contar com o amparo dos meus familiares foi crucial para trilhar esta trajetória com êxito. Obrigado pelo incentivo, amor e compreensão em todos os momentos.

Agradeço aos meus amigos que sempre me acompanharam. Obrigado por estarem nas ocasiões mais especiais da minha vida pessoal e da minha vida acadêmica. O apoio dos amigos teve grande impacto positivo na redação deste trabalho.

Agradeço ao meu orientador Vyacheslav Futorny pela sua orientação e liberdade na condução do trabalho durante os últimos 7 anos de trabalho juntos desde a iniciação científica, também sou grato pela proposta de trabalhar neste tema e pelas oportunidades oferecidas durante o vínculo que mantivemos. 



\section{Conteúdo}

Resumo

Abstract $\quad$ v

Agradecimentos vii

1 Introdução 1

2 Álgebras de Lie 5

2.1 Definições básicas . . . . . . . . . . . . . . . . . . . 6

2.1 .1 Subálgebras e ideais . . . . . . . . . . . . . 7

2.1.2 Homomorfismo de Lie . . . . . . . . . . . . . . . . 7

2.1.3 Álgebras de Lie simples . . . . . . . . . . . . . . . . . . . . . 8

2.1 .4 Somas diretas . . . . . . . . . . . . . . . . . . . . 8

2.1.5 Álgebras envelopantes universais . . . . . . . . . . . . 8

2.1.6 O Teorema de Poincaré-Birkhoff-Witt (PBW) . . . . . . . . . . . 9

2.1 .7 Álgebras graduadas . . . . . . . . . . . . . . . . . . . . . . 9

2.2 Representações de álgebras de Lie . . . . . . . . . . . . . . . . . . . 10

2.2.1 Representações e módulos . . . . . . . . . . . . . . . . . . . . 10

2.2.2 Forma de Cartan-Killing . . . . . . . . . . . . . . . . . . . . . 11

2.3 Álgebras de Lie afim . . . . . . . . . . . . . . . . . . . . . . 11

2.3.1 Álgebras de Kac-Moody e álgebras de Lie afim . . . . . . . . . . 11

2.3.2 Diagramas de Dynkin . . . . . . . . . . . . . . . . 13

3 Álgebras de Krichever-Novikov $\quad 17$

3.1 Curvas algébricas . . . . . . . . . . . . . . . . . . . 18

3.2 Superfícies de Riemann . . . . . . . . . . . . . . . . . . . . . . . . 18

3.3 Funções meromorfas . . . . . . . . . . . . . . . . . . . . . . . . . . 19

3.4 Álgebras correntes . . . . . . . . . . . . . . . . . . . . . . . . 19

3.5 A situação de Krichever-Novikov . . . . . . . . . . . . . . . . . . . . 20

3.5 .1 O caso 1-ponto . . . . . . . . . . . . . . . . 20

3.5.2 A situação de Krichever-Novikov generalizada . . . . . . . . . 20

3.6 Quasegraduação . . . . . . . . . . . . . . . . . . . . . . 21

3.7 Extensões centrais universais de álgebras de Krichever-Novikov . . . . 23

3.7.1 Complexos e cohomologia . . . . . . . . . . . . . . 23

3.7 .2 Extensões centrais . . . . . . . . . . . . . . . . . . 24

3.7.3 Álgebras multiponto de gênero zero . . . . . . . . . . . . . . . 25

3.7.4 Álgebras de gênero maior ou igual a zero . . . . . . . . . . . . . 27

3.8 Álgebras de Lie afim superelípticas . . . . . . . . . . . . . . . . . . 31 
4 Polinômios Ortogonais $\quad 39$

4.1 Polinômios ortogonais . . . . . . . . . . . . . . . . 40

4.2 Polinômios ortogonais associados . . . . . . . . . . . . . . . 42

4.3 Álgebras DJKM e polinômios ortogonais . . . . . . . . . . . . . . . . . . . 4 43

$4.3 .1 \quad$ Caso elíptico $1 \ldots \ldots \ldots$. . . . . . . . . . . . . . . 43

4.3 .2 Caso elíptico 2. . . . . . . . . . . . . . . . . . . 44

4.4 Álgebras de Krichever-Novikov superelípticas e polinômios ortogonais 45

4.4 Caso $1 \ldots \ldots \ldots \ldots \ldots \ldots$

4.4 Caso $2 \ldots \ldots \ldots \ldots \ldots \ldots \ldots$

4.4 .3 Caso $3 \ldots \ldots \ldots \ldots \ldots \ldots \ldots$

4.4 .4 Caso $4 \ldots \ldots \ldots \ldots \ldots \ldots \ldots$

4.4 .5 Equações diferenciais para os Casos 1 e $2 \ldots \ldots$. . . . . . . 48

4.4 .6 Polinômios ultraesféricos associados . . . . . . . . . . . . . 58

4.4 .7 Ortogonalidade de $P_{-1, n}$ e $P_{-3, n} \ldots \ldots \ldots$. . . . . . . 60

5 Representações de álgebras de Krichever-Novikov 63

5.1 Módulos de Verma . . . . . . . . . . . . . . . . . . . . . . 64

5.2 Módulos $\varphi$ Verma . . . . . . . . . . . . . . . . . . . 65

5.2.1 Módulos de Verma imaginários . . . . . . . . . . . . . . . . . 65

5.2.2 Módulos $\varphi$ Verma para subálgebras de Heisenberg . . . . . . . 66

5.3 Módulos irredutíveis para subálgebras de Heisenberg . . . . . . . . . . 67

5.4 A álgebra de Lie hiperelíptica . . . . . . . . . . . . . . . . . . . 6 67 67

5.5 Subálgebra de Heisenberg hiperelíptica . . . . . . . . . . . . . . . 69

5.6 Módulos $\varphi$ Verma para a subálgebra de Heisenberg hiperelíptica . . . . 70

6 Perspectivas futuras e considerações finais $\quad 73$

6.1 Perspectivas futuras . . . . . . . . . . . . . . 73

6.2 Considerações finais . . . . . . . . . . . . . . . 74

$\begin{array}{ll}\text { Bibliografia } & 75\end{array}$ 


\section{Lista de Figuras}

3.1 I. Krichever . . . . . . . . . . . . . . . . . . . . . . . . . . 17

3.2 S. Novikov . . . . . . . . . . . . . . . . . . . . . . . . 17

3.3 Esfera de Riemann . . . . . . . . . . . . . . . . . . . . . 18

3.4 Toro . . . . . . . . . . . . . . . . . . . . . . . . . . . 19

4.1 G. Szegő . . . . . . . . . . . . . . . . . . . . . . . . . . 39 

Dedicado à minha família. 



\section{Capítulo 1}

\section{Introdução}

Nas últimas décadas, as álgebras de Lie de dimensão infinita tem se provado um rico campo de pesquisa. As álgebras de Krichever-Novikov são exemplos de álgebras de Lie de dimensão infinita que foram introduzidas no estudo de teoria de cordas no espaço de Minkowski em [KN87] e [KN88]. Essas álgebras foram estudadas de forma profunda nos últimos anos por diversos autores, conforme é possível observar em [Sch14b] e nas suas referências. O objetivo deste trabalho é estudar o caso superelíptico das álgebras de Krichever-Novikov.

Seja $\mathfrak{g}$ uma álgebra de Lie complexa e de dimensão finita, e $\mathcal{G}=\mathfrak{g} \otimes \mathbb{C}\left[t, t^{-1}\right]$ a álgebra laço de $\mathfrak{g}$ com relações de comutatividade dadas por $[x \otimes f, y \otimes g]=[x, y] \otimes f g$, para $x, y \in \mathfrak{g}$ e $f, g \in \mathbb{C}\left[t, t^{-1}\right]$. Denotaremos $\hat{\mathcal{G}}$ a extensão central universal de $\mathcal{G}$, que é uma álgebra de Kac-Moody não torcida de g. Na construção da álgebra laço, podemos substituir a álgebra de polinômios de Laurent $\mathbb{C}\left[t, t^{-1}\right]$ por outra álgebra complexa, associativa e comutativa, digamos $R$, e considerar a extensão central universal de $\mathfrak{g} \otimes R$. Quando $R$ é o anel de funções meromorfas numa superfície de Riemann fixa com uma quantidade finita de polos, a álgebra $\hat{\mathcal{G}}$ é chamada álgebra laço afim de Krichever-Novikov ou álgebra corrente de Krichever-Novikov. A construção deste tipo de álgebra quando considerado o anel de funções racionais na esfera de Riemann que são regulares exceto numa quantidade finita de pontos é particularmente relevante para o estudo de estruturas de módulo tensor para álgebras de Lie afim ([KL91], [KL94]). Estas álgebras de Lie que generalizam as álgebras de Kac-Moody não torcidas, são chamadas de álgebras $N$-ponto ou multiponto e são exemplos de álgebras de Krichever-Novikov de gênero zero. Em [Bre94], Bremner descreve o centro $C$ da extensão central universal $\hat{\mathcal{G}}$ de álgebras $N$-ponto e determina sua dimensão. Quando $R$ é o anel de funções regulares sobre curvas hiperelípticas com 2, 3 ou 4 pontos removidos, é determinada uma base para $C$, e no caso em que curvas elípticas com 2 pontos removidos, é calculado o cociclo universal $\hat{\mathcal{G}} \times \hat{\mathcal{G}} \rightarrow C$. Utilizando resultados obtidos nos trabalhos de Bremner, os autores Cox e Jurisich obtém em [CJ14] uma descrição explícita da extensão central universal da álgebra corrente 3-ponto em que $\mathfrak{g}=\mathfrak{s l}(2, \mathbb{C})$ e, além disso, constroem de forma explícita realizações para estas álgebras utilizando operadores diferenciais. Em [Bre95], Bremner constrói a extensão central universal das álgebras corrente 4-ponto.

Date, Jimbo, Kashiwara e Miwa consideraram a extensão central universal de $\mathfrak{g} \otimes \mathbb{C}\left[t, t^{-1}, u\right] \operatorname{com} u^{2}=\left(t^{2}-b^{2}\right)\left(t^{2}-c^{2}\right), b \in \mathbb{C} \backslash\{-c, c\}$ no seu trabalho sobre a equação de Landau-Lifshitz em [Dat+83a]. A álgebra obtida é chamada de álgebra DJKM e é um exemplo de álgebra de Krichever-Novikov com gênero diferente de zero. As extensões centrais universais de álgebras DJKM são descritas em [CF11]. Uma realização dessas álgebras em termos de operadores diferenciais parciais são construídas em [CJ14] para $\mathfrak{g}=\mathfrak{s l}(2, \mathbb{C})$ e em [CFM14] para $\mathfrak{g}$ genérica. O estudo das extensões centrais universais de álgebras DJKM culminou em descobertas de novas famílias de polinômios ortogonais em [CFT13]. 
Álgebras de Lie afim elípticas formam outra família de álgebras de Krichever-Novikov. Estas álgebras são as extensões centrais universais de álgebras de Lie $\mathfrak{g} \otimes R$, em que $R=\mathbb{C}\left[t, t^{-1}, u\right]$ e $u^{2}=k(t) \in \mathbb{C}[t]$ é uma curva elíptica. A descrição explícita destas estruturas em termos de geradores e relações de comutatividade foi estudada por Bremner em [Bre94] e [Bre95]. No caso de álgebras de Lie da forma $\mathfrak{g} \otimes R$ em que $R$ é o anel das funções regulares definidas em uma curva algébrica com uma quantidade enumerável de pontos removidos, Bremner calculou a dimensão da extensão central universal associada, o que ofereceu ferramentas necessárias para a construção de realizações de corpos livres de álgebras afim elípticas 4-ponto em [CJ14], [CF11], [Cox16].

Álgebras de Lie afim hiperelípticas formam uma família de álgebras de KricheverNovikov com a álgebra hiperelíptica $R=\mathbb{C}\left[t, t^{-1}, u\right]$, em que $u^{2}=k(t) \in \mathbb{C}[t]$ é uma curva com gênero maior que 1 . As curvas hiperelípticas são o caso mais simples de curvas superelípticas $u^{m}=k(t), \operatorname{com} k(t) \in \mathbb{C}[t]$ e $m \geq 2$ (isto significa que toda curva elíptica ou hiperelíptica é superelíptica). As álgebras de Lie superelípticas foram estudadas recentemente por Cox, Guo, Lu and Zhao em [Cox+17]. Uma pergunta natural que emerge do contexto geométrico de curvas algébricas é:

Pergunta 1: A teoria desenvolvida para curvas hiperelípticas pode ser estendida para o caso das curvas superelípticas? (veja [BSZ15] e [MS19]).

Considerando $\mathcal{G}=\mathfrak{g} \otimes R$, em que $R$ é uma álgebra de funções superelíptica, por um resultado de Kassel [Kas84], sabemos que o centro $C$ de $\hat{\mathcal{G}}$, a extensão central universal de $\mathcal{G}$, é linearmente isomorfo à $\Omega_{R}^{1} / d R$, o espaço dos diferenciais de Kähler de $R$ módulo formas exatas. Bremner [Bre94] respondeu aos seguintes problemas sobre as álgebras de Lie elípticas:

(1) Qual é uma descrição explícita de $C$ ? Qual é a dimensão de C?

(2) Como descrever explicitamente uma base para $C$ ?

(3) Calcule o cociclo universal $\hat{\mathcal{G}} \times \hat{\mathcal{G}} \rightarrow C$ explicitamente.

Inspirado por trabalhos anteriores como os citados nesta introdução e, especialmente munido de motivação proveniente da Pergunta 1, o desenvolvimento deste texto apresenta respostas para estas perguntas para o caso de álgebras de Lie afim superelípticas $\mathfrak{g} \otimes R$, em que $R=\mathbb{C}\left[t, t^{-1}, u\right], u^{m}=\sum_{i=0}^{D} a_{i} t^{i}, a_{D}=1$, com pelo menos $a_{0}$ ou $a_{1}$ não nulo e $\sum_{i=0}^{D} a_{i} t^{i}$ sendo um polinômio sem raízes múltiplas (veja o Teorema 3.8.5 e o Teorema 3.8.10). O resultado compõe o trabalho que foi recentemente aceito para publicação em Communcations in Algebra, [San p].

Teorema 1: Os diferenciais $\overline{t^{-1} d t}$, com $\overline{t^{-1} u^{l} d t}, \ldots, \overline{t^{-D} u^{l} d t}$ (em que omitimos $\overline{t^{-D} u^{l} d t}$ se $\left.a_{0}=0\right)$, com $l \in\{1,2, \ldots, m-1\}$, formam uma base de $\Omega_{R}^{1} / d R$ que tem dimensão $D(m-1)+1$ (ou $(D-1)(m-1)+1$ se $\left.a_{0}=0\right)$. Além disso, calculamos o cociclo universal $\hat{\mathcal{G}} \times \hat{\mathcal{G}} \rightarrow C$ explicitamente.

Conforme já dito, para descrever as extensões centrais universais de álgebras DJKM são necessários quatro famílias de polinômios que aparecem como coeficientes nas fórmulas que definem as relações de comutatividade. Estes polinômios respeitam relações de recursividade obtidas em [CF11]. Duas destas famílias de polinômios são dadas em termos de integrais elípticas e as outras duas famílias são variações de polinômios ultra-esféricos. Estes polinômios são profundamente estudados em [CFT13] e lá são descritas equações diferenciais de ordem 4 satisfeitas 
por estas duas famílias elípticas, além provar que esses polinômios são ortogonais e não-clássicos. A partir do estudo dos cociclos universais das álgebras superelípticas, obtém-se famílias de polinômios. Sendo assim, é natural enunciar a seguinte pergunta:

Pergunta 2: À partir das famílias de polinômios obtidas durante o estudo das relações de comutatividade de álgebras de Krichever-Novikov do tipo superelípticas, é possível obter famílias de polinômios ortogonais não-clássicos que satisfazem equações diferenciais de ordem maior que 2 ?

Considerando o caso da curva superelíptica $u^{3}=t^{4}-2 c t+1$, são descritas famílias de polinômios ortogonais não clássicos que satisfazem determinada equação diferencial linear ordinária de quarta ordem exibindo o cálculo diretamente (veja Teorema 4.4.1, Teorema 4.4.2, Teorema 4.4.4 e Teorema 4.4.3).

Teorema 2: Há uma família de polinômios que surge nos coeficientes das relações de comutatividade para álgebras de Krichever-Novikov superelípticas, considerando o caso da curva superelíptica $u^{3}=t^{4}-2 c t+1$ e certas condições iniciais é ortogonal, não clássica e satisfaz a seguinte equação diferencial de ordem 4:

$$
\begin{aligned}
& 144\left(c^{2}-1\right)^{2} P_{n}^{(4)}+1440 c\left(c^{2}-1\right) P_{n}^{(3)} \\
& -8\left(c^{2}\left(9 n^{2}+30 n-421\right)-3 n(3 n+10)+183\right) P_{n}^{\prime \prime} \\
& -24 c\left(9 n^{2}+30 n-61\right) P_{n}^{\prime}+(n-1)(n+3)(3 n+1)(3 n+13) P_{n}=0
\end{aligned}
$$

No contexto de Teoria de Representações, as álgebras de Lie afim oferecem uma complexa gama de propriedades. As álgebras de Lie afim possuem módulos irredutíveis que contém espaços de peso de dimensão finita e infinita, sendo que a classificação de módulos irredutíveis é conhecida apenas para módulos com espaço de peso de dimensão finita e para algumas subcategorias de módulos induzidos com apenas alguns espaços de peso de dimensão finita. Inspirado por [Bek+13] consideramos subálgebras de Heisenberg de álgebras de Krichever-Novikov hiperelípticas e determinamos critérios de irredutibilidade para módulos conhecidos como módulos do tipo $\varphi$-Verma.

Seja $\mathfrak{g}$ uma álgebra de Lie afim e $\mathfrak{h}$ sua subálgebra de Cartan usual, considere $\mathbb{C} c$ o centro desta álgebra, em que $c$ é o elemento central canônico. Seja $V$ um g-módulo de peso, isto é, $V=\bigoplus_{\mu \in \mathfrak{h}^{*}} V_{\mu}$, em que $V_{\mu}=\{v \in V \mid h v=\mu(h) v$ para todo $h \in \mathfrak{h}\}$. Se $V$ é irredutível, então $c$ age como um escalar em $V$ e é chamado de nível de $V$.

Pergunta 3: Quais são os critérios de irredutibilidade para módulos $\varphi$-Verma de subálgebras de Heisenberg hiperelípticas?

Dada uma álgebra de Lie afim $\mathfrak{g}$, o subespaço $L=\mathbb{C} c \oplus \bigoplus_{n \in \mathbb{Z} \backslash\{0\}} \mathfrak{g}_{n \delta}$ é uma subálgebra de Heisenberg. Seja $\varphi: \mathbb{N} \rightarrow \pm$ uma função arbitrária definida em $\mathbb{N}$. Os seguintes subespaços são subálgebras abelianas

$$
L_{\varphi}^{ \pm}=\left(\bigoplus_{n \in \mathbb{N}, \varphi(n)= \pm} \mathfrak{g}_{n \delta}\right) \oplus\left(\bigoplus_{m \in \mathbb{N}, \varphi(m)=\mp} \mathfrak{g}_{-m \delta}\right)
$$

$\mathrm{e}$

$$
L=L_{\varphi}^{-} \oplus \mathbb{C} c \oplus L_{\varphi}^{+}
$$


é uma decomposição triangular para $L$. Seja $\mathbb{C} v$ uma representação de dimensão 1 de $\mathbb{C} c \oplus L_{\varphi}^{+}$em que $c v=a v$ para algum $a \in \mathbb{C}$ e $L_{\varphi}^{+}=0$. O módulo do tipo $\varphi$-Verma é o módulo induzido dado por

$$
M_{\mathfrak{g}, \varphi}=U(L) \otimes_{U\left(\mathbb{C} c \oplus L_{\varphi}^{+}\right)} \mathbb{C} v
$$

em que $U(A)$ denota a álgebra envelopante universal de $A$. O Teorema 5.6.2 estabelece um critério de irredutibilidade para módulos $\varphi$-Verma para subálgebras de Heisenberg hiperelípticas, respondendo à Pergunta 3. O resultado compõe o trabalho publicado em arXiv, [San17a] e generaliza os resultados obtidos em [San17b]

Teorema 3: $M_{\hat{\mathfrak{g}}, \varphi}$ é irredutível se e somente se possui nível não nulo.

Além de apresentar novos resultados, espera-se que este trabalho sirva como material de consulta para estudantes interessados em álgebras de Krichever-Novikov. Tentou-se, na medida que foi possível, manter a linguagem acessível e contemplar a maior parte das definições que não são elementares. O texto apresenta excertos históricos e notas de rodapé com a modesta intenção de - para mais do que registrar os saberes desenvolvidos durante a redação deste trabalho - permitir uma visão mais ampla dos personagens e fatos envolvidos no processo de produção científica da Matemática ao longo da História, propiciar motivação e um breve afastamento dos termos duros que inevitavelmente permeiam a maior parte das páginas. 


\section{Capítulo 2}

\section{Álgebras de Lie}

Neste capítulo percorremos definições fundamentais para o trabalho que será desenvolvido nos capítulos subsequentes. Também apresentamos alguns exemplos que serão utilizados nos próximos capítulos e resultados que serão aplicados em outros contextos. 


\subsection{Definições básicas}

Considere $\mathbb{K}$ um corpo e seja $L$ um espaço $\mathbb{K}$-vetorial

Definição 2.1.1 Uma aplicação bilinear [ , ]: $L \times L \mapsto$ L. Satisfazendo

1. Propriedade antissimétrica:

$$
\forall x \in L, \quad[x, x]=0 .
$$

2. Identidade de Jacobi ${ }^{1}$ :

$$
\forall x, y, z \in L, \quad[[x, y], z]+[[y, z], x]+[[z, x], y]=0 .
$$

é chamada de colchete de Lie (ou produto de Lie) para L.

Definição 2.1.2 O par $(L,[]$,$) é chamado de álgebra de Lie.$

Exemplo 2.1.3 Se $(A, \cdot)$ é uma álgebra associativa, então $(A,[]$,$) com o produto$

$$
[x, y]:=x \cdot y-y \cdot x
$$

como produto de Lie é uma álgebra de Lie. O produto definido em (2.1) é denominado comutador.

Exemplo 2.1.4 Considere $\mathfrak{g l}(n, K)$ álgebra formada por matrizes quadradas $n \times n$, com entradas em Ke com o comutador. Esta álgebra é denominada álgebra de Lie especial linear.

Exemplo 2.1.5 Considere End $(V)=\{\varphi: V \rightarrow V \mid \varphi$ é linear $\}$ a álgebra dos endomorfismos lineares com produto dado pela composição destas aplicações. A álgebra End $(V)$ com o comutador define uma álgebra de Lie denotada comumente por $g l(V)$.

Exemplo 2.1.6 Considere $\mathfrak{s l}(n, \mathbb{C})$, o conjunto das matrizes complexas $n \times n$ com traço 0 . Note que $\mathfrak{s l}(2, \mathbb{C})$ tem uma base formada por

$$
x=\left(\begin{array}{ll}
0 & 1 \\
0 & 0
\end{array}\right), \quad y=\left(\begin{array}{ll}
0 & 0 \\
1 & 0
\end{array}\right), \quad h=\left(\begin{array}{cc}
1 & 0 \\
0 & -1
\end{array}\right) .
$$

O conjunto $\mathfrak{s l}(2, \mathbb{C})$ com o produto

$$
[h, x]=2 x, \quad[h, y]=-2 y, \quad[x, y]=h
$$

é uma álgebra de Lie.

Exemplo 2.1.7 Suponha que a é uma álgebra de Lie sobre $\mathbb{C}$ que possui um subespaço $\mathfrak{c}$ de dimensão 1 com as seguintes propriedades

$$
\begin{aligned}
{[\mathfrak{a}, \mathfrak{a}] } & =\mathfrak{c}, \\
{[\mathfrak{a}, \mathfrak{c}] } & =(0) .
\end{aligned}
$$

A álgebra a munida de uma aplicação bilinear antissimétrica não degenerada $\psi$ em a/c é denominada álgebra de Heisenberg ${ }^{2}$.

Podemos também descrever uma álgebra de Heisenberg como sendo uma álgebra $\hat{\mathfrak{h}}$ gerada por $\left\{a_{n} \mid n \in \mathbb{Z} \backslash\{0\}\right\} \cup\{b\}$, onde b é central e $\left[a_{m}, a_{n}\right]=m \delta_{m,-n} b$.

\footnotetext{
${ }^{1}$ Carl Gustav Jacob Jacobi (1804-1851) foi um matemático alemão com contribuições em funções elípticas, dinâmica, equações diferenciais e teoria dos números.

${ }^{2}$ Werner Karl Heisenberg (1901-1976) foi um físico teórico alemão que recebeu o Nobel de física em 1932.
} 
$\delta_{i, j}$ é o delta de Kronecker ${ }^{3}$. A definição do $\delta_{i, j}$ é a seguinte:

$$
\delta_{i, j}=\left\{\begin{array}{l}
0 \text { se } i \neq j, \\
1 \text { se } i=j .
\end{array}\right.
$$

\subsubsection{Subálgebras e ideais}

Definição 2.1.8 Dada uma álgebra de Lie $(L,[$, ]), definimos que

a. Um subespaço vetorial $M$ de L é chamado de subálgebra de Lie se

$$
[M, M] \subset M \text {. }
$$

b. Um subespaço $M$ de Lé chamado de ideal (de Lie) de L se

$$
[M, L] \subset M \text {. }
$$

c. A álgebra de Lie Lé chamada de abeliana se

$$
[L, L]=\{0\} .
$$

Exemplo 2.1.9 São subálgebras de $\mathfrak{g l}(n, \mathbb{C})$

$$
\begin{aligned}
\mathfrak{s l}(n, \mathbb{C}): & =\{X \in \mathfrak{g l}(n, \mathbb{C}) \mid \operatorname{tr}(X)=0\} e \\
\mathfrak{s o}(n, \mathbb{C}): & =\left\{\left.X \in \mathfrak{g l}(n, \mathbb{C})\right|^{t} X=-X\right\}
\end{aligned}
$$

Exemplo 2.1.10 Todo ideal de Lie é também uma subálgebra de Lie. Para toda álgebra de Lie L temos os seguintes ideais.

1. O centro que é definido por

$$
C(L)=\{x \in L \mid \forall y \in L:[x, y]=0\} .
$$

2. A subálgebra derivada definida por

$$
L^{\prime}:=[L, L]:=\langle[x, y] \mid x, y \in L\rangle_{K} .
$$

\subsubsection{Homomorfismo de Lie}

Dadas duas álgebras de Lie $\left.\left(L,[,]^{(1)}\right]\right)$ e $\left(M,\left(L,[,]^{(2)}\right]\right)$, uma aplicação linear $\phi: L \rightarrow M$ é chamada de homomorfismo de Lie se respeita a estrutura da álgebra de Lie, isto é, se

$$
\forall x, y \in L \quad[\phi(x), \phi(y)]^{(2)}=\phi\left([x, y]^{(1)}\right) .
$$

O núcleo deste homomorfismo ker $\phi$ é um ideal de $L$ e a imagem deste homomorfismo $\operatorname{Im} \phi$ é uma subálgebra de $M$. Considerando $i$ como a inclusão natural, podemos obter a seguinte sequência exata curta:

$$
0 \longrightarrow \operatorname{ker} \phi \stackrel{i}{\longrightarrow} L \stackrel{\phi}{\longrightarrow} \operatorname{Im} \phi \longrightarrow 0 .
$$

Sabendo que dado um ideal J de L, o espaço quociente $L / J$ tem um produto de Lie natural dado por

$$
[x \bmod J, y \bmod J]:=[x, y] \bmod J,
$$

podemos obter também uma sequência exata curta da seguinte forma:

$$
0 \longrightarrow J \stackrel{i}{\longrightarrow} L \stackrel{v}{\longrightarrow} L / J \longrightarrow 0,
$$

em que $v$ é a projeção natural de $L$ em $L / J$.

\footnotetext{
${ }^{3}$ Leopold Kronecker(1823 - 1891) foi um matemático alemão com contribuições em álgebra, teoria dos números e no estudo de continuidade de funções.
} 


\subsection{3 Álgebras de Lie simples}

Uma álgebra de Lie $L$ é chamada de simples se, e somente se, $L$ não é abeliana e só possui ideais triviais (isto é, só possui como ideais 0 e $L$ ). Portanto, para álgebras de Lie simples, o centro e a subálgebra derivada são ideais triviais.

Uma álgebra de Lie $L$ é chamada de perfeita se $L^{\prime}=[L, L]=L$. Em particular, álgebras de Lie simples são perfeitas.

\subsubsection{Somas diretas}

Dadas duas álgebras de Lie $\left.\left(L,[,]^{(1)}\right]\right)$ e $\left(M,\left(L,[,]^{(2)}\right]\right)$, definimos a álgebra de Lie soma direta como o espaço vetorial $L \oplus M$ com o produto

$$
\left[\left(l_{1}, m_{1}\right),\left(l_{2}, m_{2}\right)\right]:=\left(\left[l_{1}, l_{2}\right],\left[m_{1}, m_{2}\right]\right)
$$

em que $l_{1}, l_{2} \in L$ e $m_{1}, m_{2} \in M$. Note que $L$ e $M$ são ideais de $L \oplus M$.

Uma álgebra de Lie de dimensão finita é chamada semisimples se $L$ for a soma direta de álgebras de Lie simples $L_{i}, i=1, \ldots, M$

$$
L=L_{1} \oplus L_{2} \oplus \cdots \oplus L_{M}
$$

Uma álgebra de Lie é chamada redutível, se $L$ for a soma de uma álgebra de Lie abeliana com uma álgebra de Lie semisimples.

\subsection{5 Álgebras envelopantes universais}

Dada uma álgebra de Lie $L$ podemos construir uma álgebra $U(L)$ associativa e com unidade de forma que sua álgebra comutadora $\left(U(L),[,]^{(1)}\right)$ possui $L$ como subálgebra. Para isso, consideramos $L$ como espaço vetorial e construímos a álgebra tensorial $T(L)$ com produto induzido pelo concatenamento dos tensores, isto é, por exemplo:

$$
(x \otimes y) \cdot(z \otimes w \otimes u):=x \otimes y \otimes z \otimes w \otimes u .
$$

Considere $J$ um ideal bilateral gerado por $\left(x_{1} \otimes x_{2}-x_{2} \otimes x_{1}-\left[x_{1}, x_{2}\right]\right) \operatorname{com} x_{1}, x_{2} \in$ $L$. A álgebra envelopante universal é definida como a álgebra associativa $U(L)$ da seguinte forma:

$$
U(L)=T(L) / J
$$

Temos a inclusão

$$
\phi: L \rightarrow U(L), \operatorname{com} x \mapsto x \bmod J .
$$

Levando em consideração a álgebra $U(L)$ definida com o produto de Lie dado pelo comutador, teremos que $\phi$ será um homomorfismo de Lie. Portanto, $L$ pode ser interpretada como uma subálgebra de $U(L)$.

É importante notar que dada uma álgebra associativa $A$ com unidade, se existir um homomorfismo de Lie

$$
\psi: L \rightarrow(A,[,])
$$

então existe um único homomorfismo de álgebras associativas

$$
\hat{\psi}: U(L) \rightarrow A \text { tal que } \hat{\psi} \circ \phi=\psi \text {. }
$$

Por outro lado, dado um homomorfismo de álgebras $\hat{\psi}: U(L) \rightarrow A$, existe um homomorfismo de Lie $\psi=\hat{\psi} \circ \phi, \psi: L \rightarrow(A,[]$,$) . Portanto podemos dizer que a$ álgebra envelopante universal obedece a seguinte relação universal. 


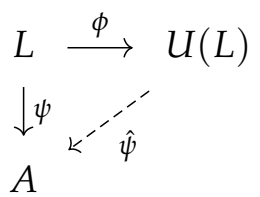

Exemplo 2.1.11 A álgebra envelopante universal $U(\mathfrak{s l}(2, \mathbb{C}))$ é a álgebra gerada por e, $f, h$ com relações dadas por

$$
h e-e h=2 e, \quad h f-f h=-2 f, \quad \text { ef }-f e=h .
$$

\subsubsection{O Teorema de Poincaré-Birkhoff-Witt (PBW)}

O Teorema a seguir tem importantes aplicações em Teoria de Representações, seu nome é uma homenagem aos matemáticos Poincaré ${ }^{4}$, Garrett Birkhoff ${ }^{5}$ e Ernst Witt ${ }^{6}$.

Teorema 2.1.12 (PBW) Seja $\mathfrak{g}$ uma álgebra de Lie com uma $\mathbb{K}$-base $\left\{x_{j} \mid j \in J\right\}$ indexada num conjunto totalmente ordenado J. Seja $U(\mathfrak{g})$ a álgebra envelopante universal de $\mathfrak{g} e$ i a aplicaçao $\mathbb{K}$-linear canônica de $\mathfrak{g}$ em $U(\mathfrak{g})$, então

$$
i\left(x_{j_{1}}\right) i\left(x_{j_{2}}\right) \cdots i\left(x_{j_{n}}\right)
$$

onde $j_{1} \leq j_{2} \leq \cdots \leq j_{n}, n \in \mathbb{Z}_{>0}$, junto com $1 \in \mathbb{K}$ forma uma base de $U(\mathfrak{g})$.

Aqui $i$ denota um homomorfismo de álgebras $i: \mathfrak{g} \mapsto U(\mathfrak{g})$ tal que

(i) $i([x, y])=i(x) i(y)-i(y) i(x)$, para todo $x, y \in \mathfrak{g}$.

(ii) Dada uma álgebra associativa $A$ e qualquer homomorfismo de álgebras de Lie $f: \mathfrak{g} \rightarrow \operatorname{Lie}(A)$ existe um único homomorfismo de álgebras $f^{\prime}: U(\mathfrak{g}) \mapsto A$ tal que o diagrama

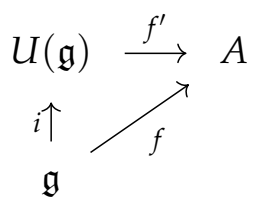

é comutativo.

\subsection{7 Álgebras graduadas}

Seja $V$ um $\mathbb{K}$-espaço vetorial de dimensão finita e $Q$ um grupo abeliano e aditivo. Entenderemos por uma graduação de $V$ por $Q$ uma família $\left\{V^{\alpha}\right\}_{\alpha \in Q}$ de subespaços de $V$ tais que $V=\bigoplus_{\alpha \in Q} V^{\alpha}$. Diremos também que $V$ é graduado por $Q$ ou $Q$-graduado. Cada subespaço $V^{\alpha}$ é um subespaço homogêneo de grau $\alpha$ e cada elemento $v \in V^{\alpha}$ é dito homogêneo (de grau $\alpha$ ). Estenderemos esta noção para o caso de álgebras.

Definição 2.1.13 Seja $\mathcal{L}$ uma álgebra de Lie. Diremos que $\mathcal{L}$ é uma álgebra de Lie graduada $s e$

\footnotetext{
${ }^{4}$ Jules Henri Poincaré (1854 - 1912) foi um matemático, físico e filósofo da ciência francês.

${ }^{5}$ Garrett Birkhoff $(1911$ - 1996) foi um matemático estadunidense.

${ }^{6}$ Ernst Witt (1911 - 1991) foi um matemático alemão conhecido por suas contribuições em álgebra.
} 
1. L é um espaço vetorial soma direta

$$
\mathcal{L}=\bigoplus_{n \in \mathbb{Z}} \mathcal{L}_{n}
$$

2. Para todo $m, n \in \mathbb{Z}$,

$$
\left[\mathcal{L}_{n}, \mathcal{L}_{m}\right] \subseteq \mathcal{L}_{m+n}
$$

Os elementos $\mathcal{L}_{n}$ são chamados de elementos homogêneos de grau ne $\mathcal{L}_{n}$ é chamado de subespaço homogêneo de grau $n$.

Uma graduação do tipo $L=L \oplus(0)$ é denominada graduação trivial. Toda álgebra admite tal tipo de graduação.

\subsection{Representações de álgebras de Lie}

\subsubsection{Representações e módulos}

Dada uma álgebra de Lie $L$ e um $\mathbb{K}$-espaço vetorial $\mathrm{V}$, uma ação de Lie de $L$ em $V$ é dada por uma aplicação bilinear

$$
L \times V \rightarrow V, \quad(x, v) \mapsto x \cdot v,
$$

tal que $\forall x, y, \in L, \forall v \in V$ teremos que

$$
[x, y] \cdot v=x \cdot(y \cdot v)-y \cdot(x \cdot v) .
$$

Denominamos $V$ de módulo de Lie sobre $L$ (ou simplesmente $L$-módulo) com estrutura de módulo dada pela ação.

De forma equivalente, podemos interpretar uma ação de Lie de outra forma. Fixando $x \in L$, considere aplicação $v \mapsto \phi_{x}(v):=x \cdot v$, em que, naturalmente, $\phi_{x} \in$ $\operatorname{End}(V)$. A condição (2.3) é equivalente a aplicação $\phi: L \rightarrow g l(V)$ em que $x \mapsto \phi_{x}$ é um homomorfismo de Lie. Podemos dizer que a estrutura de um módulo de Lie em $V$ corresponde a um homomorfismo de Lie $\phi: L \rightarrow g l(V)$. Este homomorfismo é chamado de representação de Lie e $V$ é chamado de espaço da representação.

Exemplo 2.2.1 Toda álgebra de Lie pode operar em si mesma através da seguinte aplicação:

$$
\text { ad }: x \mapsto\left\{\begin{array}{l}
L \rightarrow g l(L) \\
y \mapsto a d_{x}(y):=[x, y] .
\end{array}\right.
$$

A aplicação ad é um homomorfismo de Lie e esta representação é chamada de representação adjunta. Note que o kernel de uma representação adjunta é o centro da álgebra de Lie.

Considere $\mathfrak{s l}(2)$ conforme visto no Exemplo 2.1.6 considerando a base $x, y, h$, temos que

$$
a d(h)=\left(\begin{array}{ccc}
2 & 0 & 0 \\
0 & 0 & 0 \\
0 & 0 & -2
\end{array}\right), \quad a d(x)=\left(\begin{array}{ccc}
0 & -2 & 0 \\
0 & 0 & 1 \\
0 & 0 & 0
\end{array}\right), \quad a d(y)=\left(\begin{array}{ccc}
0 & 0 & 0 \\
-1 & 0 & 0 \\
0 & 2 & 0
\end{array}\right) .
$$

Definição 2.2.2 Seja $V$ um L-módulo. Dizemos que $V$ é um módulo irredutível se para todo $W \subset V$, tal que $U(L) W \subset V$, termos que $W=(0)$ ou $W=V$.

Definição 2.2.3 Seja $V$ um L-módulo. Dizemos que $V$ é um módulo indecomponível se não existem $W_{1}, W_{2} \subset V$ não triviais (isto é, $W_{1}, W_{2} \neq 0$ e $W_{1}, W_{2} \neq V$ ) invariantes em relação a $L$ tais que $V=W_{1} \oplus W_{2}$. 
Note que todo módulo irredutível é indecomponível, no entanto nem todo módulo indecomponível é irredutível.

Definição 2.2.4 Se $\mathcal{L}$ é uma álgebra graduada e $\mathcal{M}$ é um $\mathcal{L}$-módulo, dizemos que $\mathcal{M}$ é um módulo graduado se

1. M é um espaço vetorial soma direta

$$
\mathcal{M}=\bigoplus_{n \in \mathbb{Z}} \mathcal{M}_{n}
$$

2. Para todo $m, n \in \mathbb{Z}$,

$$
\mathcal{L}_{n} \cdot \mathcal{M}_{m} \subseteq \mathcal{M}_{m+n}
$$

Os elementos $\mathcal{M}_{n}$ são chamados de elementos homogêneos de grau n e $\mathcal{M}_{n}$ é chamado de subespaço homogêneo de grau $n$.

\subsubsection{Forma de Cartan-Killing}

Definição 2.2.5 Uma aplicação simétrica bilinear da forma $\beta: L \times L \rightarrow K$ é chamada de invariante se

$$
\beta([x, y], z)=\beta(x,[y, z])
$$

$\forall x, y \in L$

Para álgebras de Lie de dimensão finita, a forma de Cartan-Killing é a aplicação

$$
\beta(x, y):=\operatorname{tr}\left(a d_{x} \circ a d_{y}\right),
$$

em que $x, y \in L$. A forma de Cartan-Killing é uma aplicação simétrica bilinear e invariante.

Se uma álgebra de Lie é simples, então a forma de Cartan-Killing é não-degenerada.

Exemplo 2.2.6 Considere o Exemplo 2.2.1. Teremos que

$$
\beta=\left(\begin{array}{lll}
0 & 0 & 4 \\
0 & 8 & 0 \\
4 & 0 & 0
\end{array}\right)
$$

é a forma de Killing que tem determinante igual a -128 e, é não degenerada.

\section{3 Álgebras de Lie afim}

\subsection{1 Álgebras de Kac-Moody e álgebras de Lie afim}

Seja $A=\left(a_{i j}\right)_{i, j=1}^{n}$ uma matriz complexa, $n \times n$ de posto $\ell$. Vamos associá-la a uma álgebra de Lie complexa $\mathfrak{g}(A)$. A matriz $A$ é chamada de matriz de Cartan generalizada se satisfizer as seguintes condições.

$$
\begin{gathered}
a_{11}=2 \text { para } i=1, \ldots, n ; \\
a_{i j} \text { são inteiros não positivos para } i \neq j ; \\
a_{i j}=0 \text { implica que } a_{j i}=0 .
\end{gathered}
$$


Exemplo 2.3.1 A matriz $G=\left(\begin{array}{cc}2 & -3 \\ -1 & 2\end{array}\right)$ é uma matriz de Cartan.

Uma realização da matriz $A$ é uma tripla $\left(\mathfrak{h}, \Pi, \Pi^{\vee}\right)$ em que $\mathfrak{h}$ é uma espaço vetorial complexo, $\Pi=\left\{\alpha_{1}, \ldots, \alpha_{n}\right\} \subset \mathfrak{h}^{*}$ e $\Pi^{\vee}=\left\{\alpha_{1}^{\vee}, \ldots, \alpha_{n}^{\vee}\right\}$ são conjuntos indexados em $\mathfrak{h}^{*}$ e $\mathfrak{h}$ satisfazendo as seguintes condições

os conjuntos $\Pi$ e $\Pi^{\vee}$ são linearmente independentes;

$$
\begin{gathered}
\left\langle\alpha_{i}^{\vee}, \alpha_{j}\right\rangle=a_{i j},(i, j=1, \ldots, n) ; \\
n-\ell=\operatorname{dim} \mathfrak{h}-n .
\end{gathered}
$$

Proposição 2.3.2 ([Kac94], Proposição 1.1) Existe uma única realização, a menos de isomorfismos, para cada matriz $n \times n$. O isomorfismo é único se $\operatorname{det} A \neq 0$

Dizemos que o conjunto $\Pi$ é a base de raízes e que $\Pi^{\vee}$ é a base de coraízes. Os elementos de $\Pi$ são chamados de raízes simples e os elementos de $\Pi^{\vee}$ são chamados de coraízes simples . Podemos também definir

$$
Q=\sum_{i=1}^{n} \mathbb{Z} \alpha_{i}, \quad Q_{+}=\sum_{i=1}^{n} \mathbb{Z}_{+} \alpha_{i} .
$$

O reticulado $Q$ é chamado de reticulado de raízes. Para cada $\alpha=\sum_{i} k_{i} \alpha_{i} \in Q$, o número ht $\alpha:=\sum_{i} k_{i}$ é chamado de peso de $\alpha$. O espaço $Q_{+}$pode nos ajudar a introduzir uma relação de ordem parcial $\geq \mathrm{em} \mathrm{h}^{*}$ definindo que $\lambda \geq \mu$ se $\lambda-\mu \in$ $Q_{+}$.

Seja $A=\left(a_{i j}\right)$ uma matriz complexa $n \times n$ e $\left(\mathfrak{h}, \Pi, \Pi^{\vee}\right)$ uma realização de $A$. Vamos introduzir uma álgebra de Lie auxiliar $\tilde{\mathfrak{g}}(A)$ com geradores $e_{i}, f_{i}(i=1, \ldots, n)$ e $\mathfrak{h}$, e as seguintes relações:

$$
\begin{array}{rlrl}
{\left[e_{i}, f_{j}\right]} & =\delta_{i j} \alpha_{i}^{\vee}, & (i, j=1, \ldots, n), \\
{\left[h, h^{\prime}\right]} & =0, & & \left(h, h^{\prime} \in \mathfrak{h}\right), \\
{\left[h, e_{i}\right]} & =\left\langle\alpha_{i}, h\right\rangle e_{i}, & \\
{\left[h, f_{i}\right]} & =-\left\langle\alpha_{i}, h\right\rangle f_{i} & (i=1, \ldots, n ; h \in \mathfrak{h}) .
\end{array}
$$

Pela unicidade da realização de $A$, garantida pela Proposição 2.3.2, é claro que $\tilde{\mathfrak{g}}(A)$ depende apenas de $A$.

Denotaremos por $\tilde{\mathfrak{n}}_{+}$(respectivamente por $\tilde{\mathfrak{n}}_{-}$) a subálgebra de $\tilde{\mathfrak{g}}(A)$ gerada por $e_{1}, \ldots, e_{n}$ (respectivamente $f_{1}, \ldots, f_{n}$ ). Temos que

Teorema 2.3.3 ([Kac94], Teorema 1.2) $\quad$ 1. $\tilde{\mathfrak{g}}(A)=\tilde{\mathfrak{n}}_{-} \oplus \mathfrak{h} \oplus \tilde{\mathfrak{n}}_{+}$como espaços vetoriais.

2. $\tilde{\mathfrak{n}}_{+} e \tilde{\mathfrak{n}}_{-}$são livremente gerados respectivamente por $e_{1}, \ldots, e_{n}$ e $f_{1}, \ldots, f_{n}$.

3. Com respeito a $\mathfrak{h}$ a álgebra $\tilde{\mathfrak{g}}(A)$ tem uma decomposição em espaços de raízes

$$
\tilde{\mathfrak{g}}(A)=\left(\bigoplus_{\substack{\alpha \in Q_{+} \\ \alpha \neq 0}} \tilde{\mathfrak{g}}_{-\alpha}\right) \oplus \mathfrak{h} \oplus\left(\sum_{\substack{\alpha \in Q_{+} \\ \alpha \neq 0}} \tilde{\mathfrak{g}}_{\alpha}\right)
$$

em que $\tilde{\mathfrak{g}}_{\alpha}=\{x \in \tilde{\mathfrak{g}}(A) \mid[h, x]=\alpha(h) x\}, \operatorname{dim} \tilde{\mathfrak{g}}_{\alpha}<\infty e \tilde{\mathfrak{g}}_{\alpha} \subset \tilde{\mathfrak{n}}_{ \pm}$para $\alpha_{ \pm} \in Q_{+}$.

4. Existe uma único ideal maximal $\tau$ em $\mathfrak{\mathfrak { g }}(A)$ dentre os ideais intersectando $\mathfrak{h}$ trivialmente. Além disso,

$$
\tau=\left(\tau \cap \tilde{\mathfrak{n}}_{-}\right) \oplus\left(\tau \cap \tilde{\mathfrak{n}}_{+}\right) .
$$


Sejam $\tilde{\mathfrak{n}}_{+}$e $\tilde{\mathfrak{n}}_{-}$espaços livremente gerados respectivamente por $e_{1}, \ldots, e_{n}$ e $f_{1}, \ldots, f_{n}$. O Teorema 2.3.3 nos garante que existe uma decomposição para $(\tilde{\mathfrak{g}})(A)$ como espaço vetorial. Esta decomposição é chamada de decomposição triangular de $(\tilde{\mathfrak{g}})(A)$.

Exemplo 2.3.4 Se $\mathfrak{g}=\mathfrak{s l}(n, \mathbb{C}), n>1$, considere $\mathfrak{g}_{+}$a subálgebra das matrizes triangulares estritamente superiores $e \mathfrak{g}_{-}$a subálgebra das matrizes triangulares estritamente inferiores (isto é, matrizes com entradas $\left(a_{i j}\right)$ não nulas exclusivamente quando $i>j$ ). Considere $\hat{\mathfrak{h}}$ a subálgebra das matrizes diagonais em $\mathfrak{g l}(n, \mathbb{C})$ e considere $\mathfrak{h}=\hat{\mathfrak{h}} \cap \mathfrak{s l}(n, \mathbb{C})$. Teremos que $\mathfrak{s l}(n, \mathbb{C})=\mathfrak{g}_{-} \oplus \mathfrak{h} \oplus \mathfrak{g}_{+}$é uma decomposição triangular de $\mathfrak{g}$.

Considere $A$ uma matriz $n \times n$. Seja $\left(\mathfrak{h}, \Pi, \Pi^{\vee}\right)$ uma realização de $A$ e seja $\tilde{\mathfrak{g}}(A)$ a álgebra de Lie com geradores $e_{i}, f_{i}(i=1, \ldots, n)$ e $\mathfrak{h}$, com relações definidas por (2.5). Utilizando o Teorema 2.3.3, temos que a aplicacão $\mathfrak{h} \rightarrow \tilde{\mathfrak{g}}(A)$ é injetiva. Seja $\tau$ o ideal maximal de $\tilde{\mathfrak{g}}(A)$ que intersecta $\mathfrak{h}$ trivialmente (conforme o Teorema 2.3.3) e definemos

$$
\mathfrak{g}(A)=\tilde{\mathfrak{g}}(A) / \tau
$$

A matriz $A$ é chamada de matriz de Cartan da álgebra de Lie $\mathfrak{g}(A)$ e $n$ é chamado de posto de $\mathfrak{g}(A)$.

A álgebra de Lie $\mathfrak{g}(A)$ cuja matriz de Cartan é generalizada é chamada de álgebra de Kac-Moody.

A subálgebra $\mathfrak{h}$ de $\mathfrak{g}(A)$ é chamada de subálgebra de Cartan. Os elementos $e_{i}, f_{i}$ $(i=1, \ldots, n)$ são chamados de geradores Chevalley ${ }^{7}$.

Quando uma matriz de Cartan generalizada é tal que todos os seus menores principais são positivos e $\operatorname{det} A=0$ ( $A$ é automaticamente indecomponível) é chamada de matriz de Cartan afim. A álgebra de Kac-Mody associada a uma matriz de Cartan afim é chamada de álgebras de Lie afim. Toda álgebra de Lie afim possui uma álgebra de Heisenberg como subálgebra.

\subsubsection{Diagramas de Dynkin}

Uma forma eficiente e visual de representar as matrizes de Cartan é utilizando um grafo $\Gamma$, isto é, um conjunto finito de vértices conectados por um certo número de arestas. Assumindo que $\Gamma$ é conexo (todo ponto pode ser conectado a outro por um caminho de arestas) e que não possui auto-laços (arestas de um vértice para si mesmo), suponha que os vértices de $\Gamma$ são numerados por inteiros $1, \ldots, N$. Então podemos relacionar a $\Gamma$ uma matriz $R_{\Gamma}=\left(r_{i j}\right)$ que é $N \times N$, em que $r_{i j}$ é o número de vértices conectando os pontos $i$ e $j$. Essa matriz é obviamente simétrica e é chamada de matriz de adjacência.

Defina a matriz $A_{\Gamma}=2 I-R_{\Gamma}$, em que $I$ é a matriz identidade. $\Gamma$ é chamado Diagrama de Dynkin se $A_{\Gamma}$ é positiva definida, isto é, todos seus menores principais são positivos. Sendo assim, toda matriz de Cartan pode ser representada por um diagrama de Dynkin.

Diagramas de Dynkin aparecem em diversas áreas da Matemática como, por exemplo, na teoria de singularidades, álgebras de Lie, teoria de representações, geometria algébrica e Física-Matemática.

$\Gamma$ é um diagrama de Dynkin se, e somente se, é um dos seguintes grafos:

$$
A_{N}: \circ-\circ \cdots \circ-\circ-\circ
$$

\footnotetext{
${ }^{7}$ Claude Chevalley (1909 - 1984) foi um matemático francês com contribuições em teoria dos números, geometria algébrica, teoria dos corpos de classes, teoria dos grupos finitos e teoria dos grupos algébricos. Foi membro fundador do grupo Nicolas Bourbaki.
} 


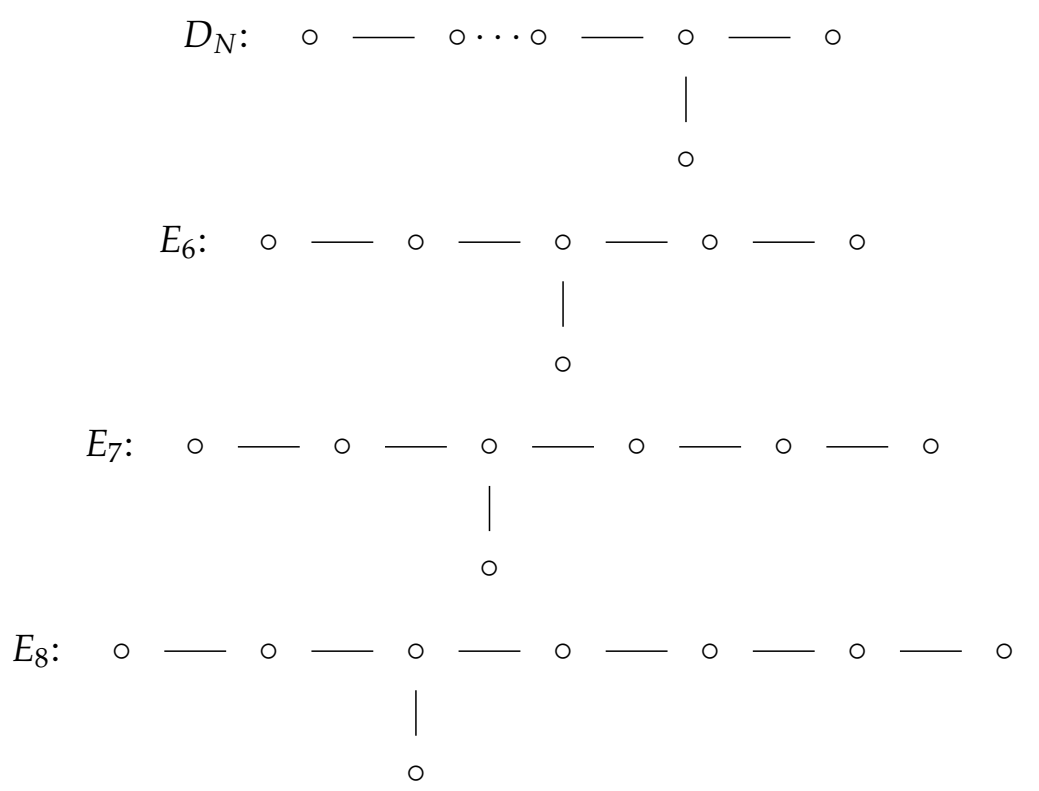

Considere $A=\left(a_{i j}\right)_{i, j=l}^{n}$ uma matiz de Cartan generalizada. Podemos associar a $A$ um grafo $S(A)$, chamado diagrama de Dynkin de $A$ da seguinte forma. Se $a_{i j} a_{i j} \leq 4$ e $\left|a_{i j}\right| \geq\left|a_{j i}\right|$, os vértices $i$ e $j$ são conectados por $\left|a_{i j}\right|$ linhas e essas linhas são dotadas de uma seta apontando para $i$ se $\left|a_{i j}\right|>1$. Se $a_{i j} a_{j i}>4$, os vértices $i$ e $j$ são conectados por uma linha em negrito com um par ordenado de inteiros $\left|a_{i j}\right|,\left|a_{j i}\right|$. Note que $A$ é indecomponível se e somente se $S(A)$ é um grafo conexo. Além disso $A$ determina o diagrama de Dynkin $S(A)$ de maneira que diremos que $S(A)$ será dito afim ou finito se $A$ for desse tipo.

Exemplo 2.3.5 Considere $G=\left(\begin{array}{cc}2 & -3 \\ -1 & 2\end{array}\right)$. Note que $a_{i j} a_{i j} \leq 4 e\left|a_{i j}\right| \geq\left|a_{j i}\right|$, portanto os vértices 1 e 2 devem ser conectados por $\left|a_{i j}\right|=3$ linhas e essas linhas devem ser dotadas de uma seta apontando para 2.

O diagrama de Dynkin associado a $G$ é

$$
\stackrel{\circ}{\Rightarrow} \underset{2}{\circ}
$$

É possível apresentar os diagramas de Dynkin de todas as matrizes de Cartan generalizadas do tipo finito. Antes de apresentar, é importante estabelecer que:

1. Os vértices dos diagramas a seguir são rotulados pelos símbolos $\alpha_{1}, \ldots, \alpha_{l}$;

2. Cada diagrama $X_{l}^{(1)}$ é obtido do diagrama $X_{l}$ adicionando um vértice, rotulado por $\alpha_{0}$, mantendo o restante dos rótulos já existentes nos vértices originais; Os números entre parênteses na tabela a seguir representam $\operatorname{det} A$;

3. Os rótulos numéricos nas tabelas a seguir são os coeficientes de uma dependência linear entre as colunas de $A$. 
Teorema 2.3.6 ([Kac94], p. 43) Os diagramas de Dynkin de todas as matrizes de Cartan generalizadas do tipo finito são:

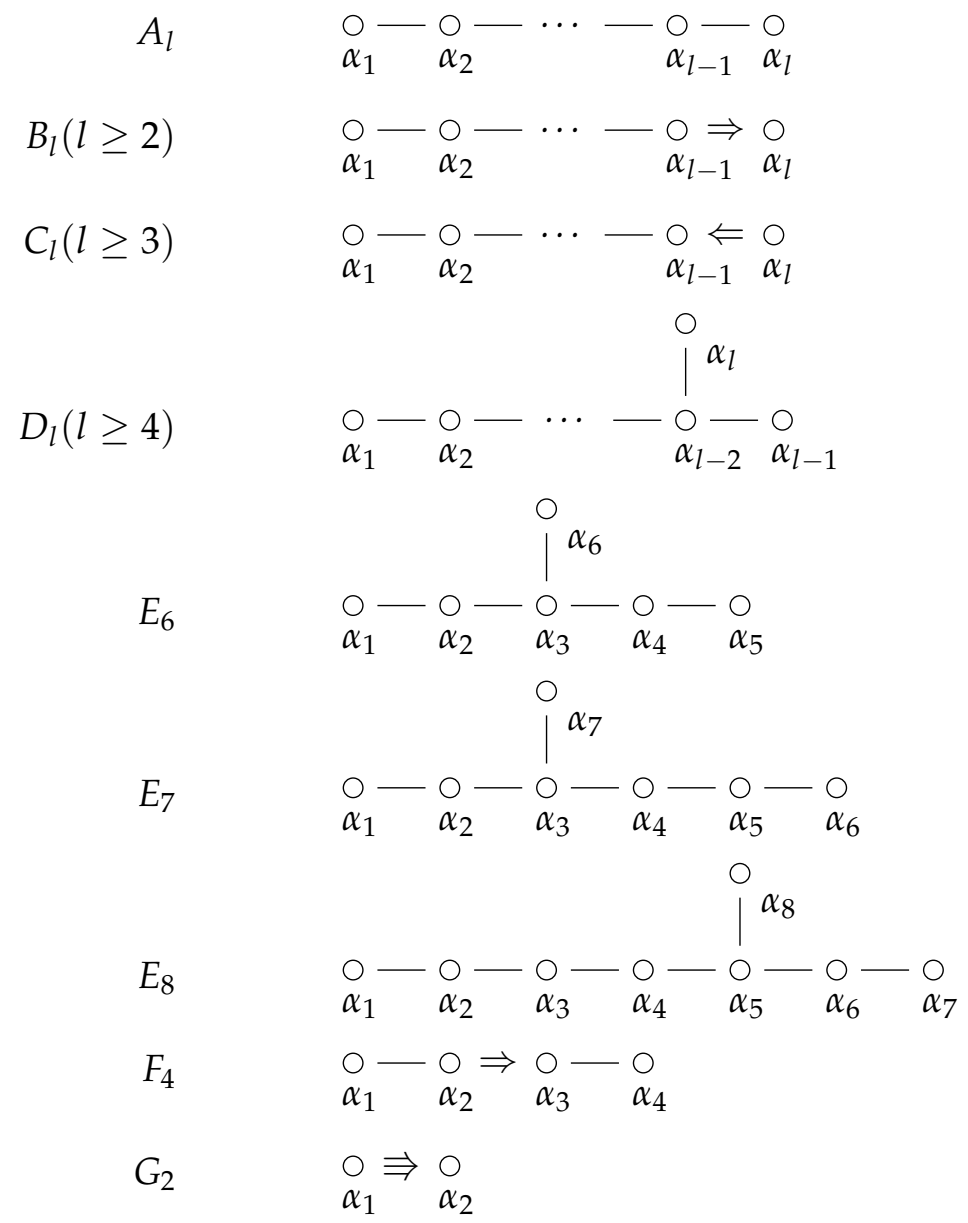

Teorema 2.3.7 ([Kac94], pp. 44 - 45) Os diagramas de Dynkin de todas as matrizes de Cartan generalizadas afim são:

$$
\begin{aligned}
& \begin{array}{ll}
A_{1}^{(1)} & 0 \Longleftrightarrow 0 \\
& 1
\end{array} \\
& A_{l}^{(1)}(l \geq 2) \\
& \underset{1}{0} \begin{array}{c}
0-\cdots-0 \\
1
\end{array}-0 \\
& B_{l}^{(1)}(l \geq 3) \\
& \stackrel{\circ}{\circ} \underset{1}{\circ}-\underset{2}{\circ}-\cdots-\underset{2}{\circ} \Rightarrow \begin{array}{l}
\circ \\
2
\end{array} \\
& C_{l}^{(1)}(l \geq 2) \\
& \begin{array}{l}
\circ \\
1
\end{array} \underset{2}{\circ}-\cdots-\bigcirc \Leftarrow \frac{0}{2} \\
& D_{l}^{(1)}(l \geq 4) \\
& { }_{1}^{\circ-}{ }_{2}^{\circ}-\circ-\cdots-\frac{\left.\right|_{2} ^{1}}{\circ}-{ }_{2}^{\circ}
\end{aligned}
$$




$$
\begin{aligned}
& G_{2}^{(1)} \quad \begin{array}{r}
\circ-\circ \\
1
\end{array} 2 \begin{array}{c}
\circ \\
3
\end{array}
\end{aligned}
$$

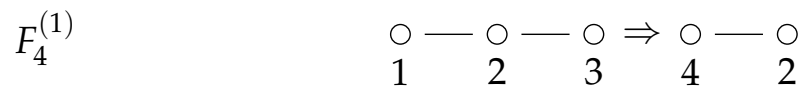

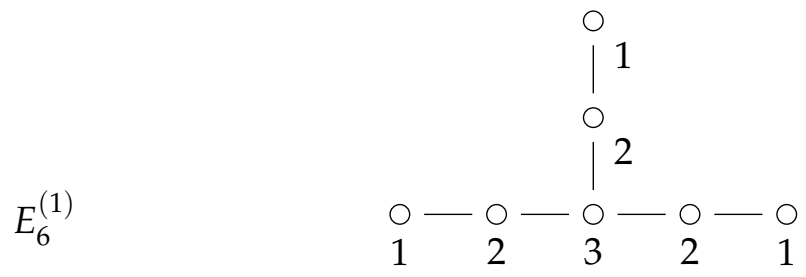

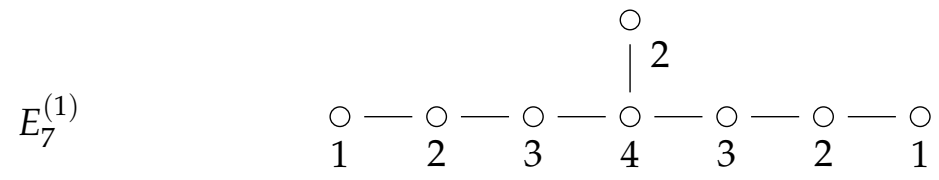

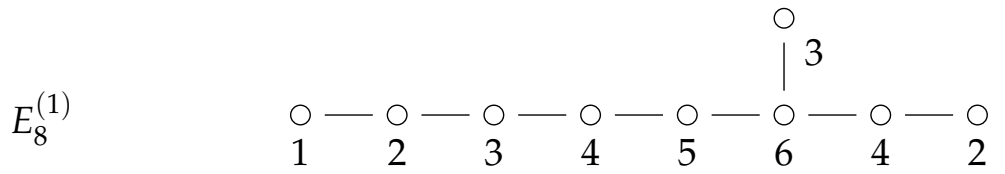

$$
\begin{aligned}
& A_{2}^{(2)} \quad \begin{array}{l}
2 \\
\circ \\
\alpha_{0}
\end{array} \\
& A_{2 l}^{(2)}(l \geq 2)
\end{aligned}
$$

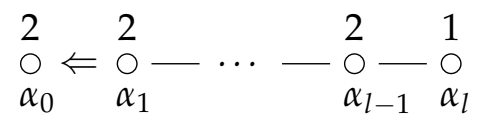

$$
\begin{aligned}
& A_{2 l-1}^{(2)}(l \geq 3)
\end{aligned}
$$

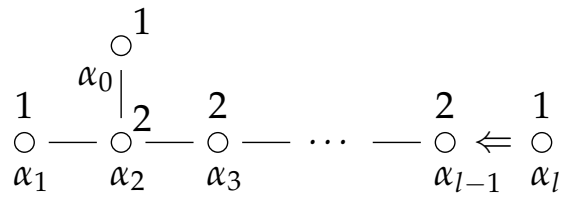

$$
\begin{aligned}
& D_{l+1}^{(2)}(l \geq 2)
\end{aligned}
$$

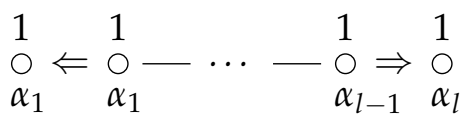

$$
\begin{aligned}
& E_{6}^{(2)} \\
& \stackrel{1}{\circ}-\stackrel{2}{\circ}-\stackrel{3}{\circ} \underset{\alpha_{0}}{\circ} \stackrel{2}{\circ} \underset{\alpha_{2}}{\circ}-\stackrel{1}{\circ} \\
& D_{4}^{(3)}
\end{aligned}
$$

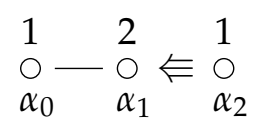

Uma álgebra de Kac-Moody afim associada à uma matriz de Cartan generalizada do tipo $X_{l}^{(1)}$ é denominada álgebra de Kac-Moody afim não torcida. 


\section{Capítulo 3}

\section{Álgebras de Krichever-Novikov}

Há vários objetos em Matemática que recebem o último sobrenome do matemático russo Igor Moiseevich Krichever (1950 - ). Durante seu doutorado, Igor foi orientado pelo matemático, também russo, Sergei Petrovich Novikov (1938 - ), laureado com a Medalha Fields em 1970. Os dois estudiosos firmaram uma duradoura parceria de colaboração e foi trabalhando em conjunto que compuseram as álgebras que hoje recebem seus nomes.

As álgebras de Krichever-Novikov são exemplos de álgebras de Lie de dimensão infinita cujos componentes são objetos meromorfos numa superfície de Riemann compacta que são holomorfos fora de um conjunto fixo de pontos. Estas álgebras generalizam as álgebras de Virasoro $^{1}$. A álgebra de Virasoro tem papel extremamente importante em álgebra pois, além de aparecer em Matemática, também tem relevância em Física. Foi estudando aplicações da álgebra de Virasoro que Krichever e Novikov deram um passo além do que já era bem estabelecido e em 1987 publicaram [KN87] apresentando as estruturas que hoje são conhecidas por álgebras de Krichever-

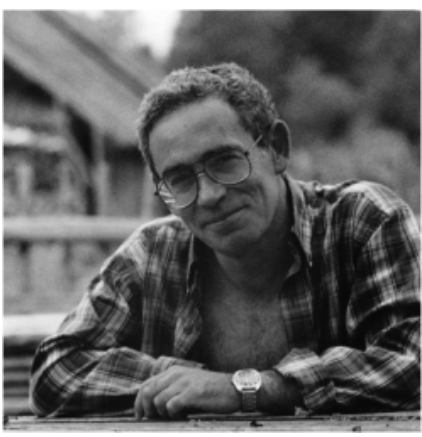

FIGURA 3.1: I. Krichever Novikov.

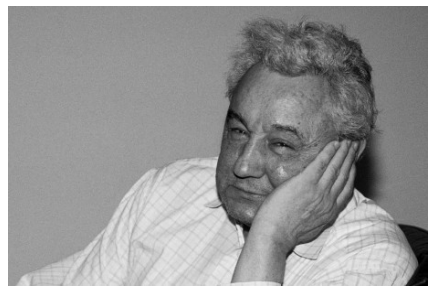

FIGURA 3.2: S. Novikov

Estas álgebras tem sido objeto de estudo de matemáticos e físicos nas últimas décadas. Do ponto de vista matemático, Martin Schlichenmaier destaca-se por ter desenvolvido a teoria das álgebras de Krichever-Novikov em contextos mais generalizados (veja, por exemplo, [Sch90b], [Sch90a], [Sch03], [Sch07]) e também por ter contribuído com a publicação de um livro ([Sch14b]) em que organiza os progressos alcançados nos últimos anos. As álgebras de Krichever-Novikov continuam a despertar interesse da academia e ainda há muitas perguntas sobre as propriedades destas estruturas.

Este capítulo apresenta como resultado principal uma descrição, em termos de geradores e relações, da extensão central universal da álgebra de Lie afim superelíptica de dimensão infinita $\mathfrak{g} \otimes R$ com a álgebra de Lie simples de dimensão finita $\mathfrak{g}$ e anel de coordenadas $R=\mathbb{C}\left[t, t^{-1}, u\right]$, em que $u^{m}=p(t)$ e $p(t)$ é um polinômio com raízes distintas. Este resultado revela, para o caso superelíptico, a descrição que era conhecida exclusivamente para o caso hiperelíptico das álgebras de KricheverNovikov (veja [Bre94]) e será publicado em Communications in Algebra ([San p]).

\footnotetext{
${ }^{1}$ Miguel Ángel Virasoro (1940 - ) é um físico argentino que foi presidente do Centro Internacional de Física Teórica Abdus Salam (ICTP).
} 


\subsection{Curvas algébricas}

Uma curva algébrica plana afim é conjunto de zeros de um polinômio de duas variáveis. Uma curva algébrica projetiva é o conjunto de zeros de um polinômio homogêneo de três variáveis no plano projetivo. De forma mais genérica, uma curva algébrica, é uma variedade algébrica de dimensão 1.

Exemplo 3.1.1 Uma curva elíptica em $\mathbb{C}$ é formada pelas soluções $(x, y)$ de

$$
y^{2}=x^{3}+a x+b
$$

em que $a, b \in \mathbb{C}$. Além disso, a curva deve ser do tipo não singular, isto é, não deve possuir auto-interseções ou cúspides (ponto na curva em que um ponto em movimento deve reverter sua direção: "bico").

Exemplo 3.1.2 Uma curva superelíptica em $\mathbb{C}$ é uma curva algébrica definida por uma equação da forma

$$
y^{m}=f(x)
$$

em que $m \geq 2$ é um inteiro e $f$ é um polinômio com grau $d \geq 3$ e coeficientes em $\mathbb{C}$. Podemos dizer que uma curva superelíptica é uma curva projetiva suave definida por esta equação. Note que se $m=2$ ed $=3$, obtemos uma curva elíptica. Caso $m=2$ e d $\geq 5$ a curva obtida é chamada de curva hipereliptica.

\subsection{Superfícies de Riemann}

Uma superfície de Riemann ${ }^{2} \Sigma$ é uma variedade complexa, 1-dimensional. A menos de menção contrária, neste trabalho consideraremos que as superfícies de Riemann são compactas. As superfícies de Riemann, do ponto de vista topológico, podem ser classificadas pelo seu gênero $g=g(\Sigma)$ que pode ser compreendido, de forma intuitiva, como a quantidade de "buracos"que há em $\Sigma$.

Exemplo 3.2.1 A esfera de Riemann é a única superfície de Riemann compacta de gênero 0. Iremos denotar esta superfície por $S^{2}$.

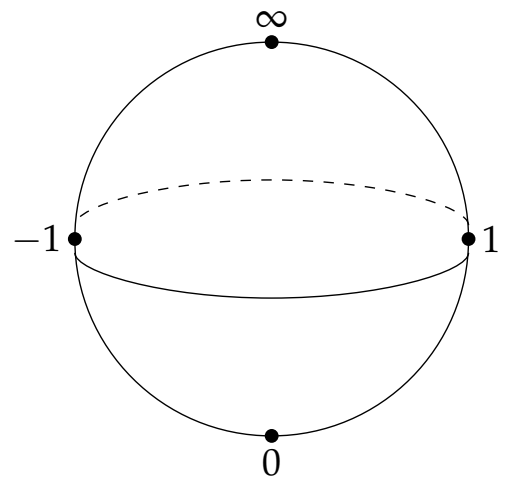

FIGURA 3.3: Esfera de Riemann

Exemplo 3.2.2 O toro é uma superfície de Riemann de gênero 1.

Superfícies de Riemann compactas podem ser descritas como curvas projetivas suaves em $\mathbb{C}$.

\footnotetext{
${ }^{2}$ Georg Friedrich Bernhard Riemann (1826 - 1866) foi um matemático alemão com contribuições fundamentais para a análise e a geometria diferencial
} 


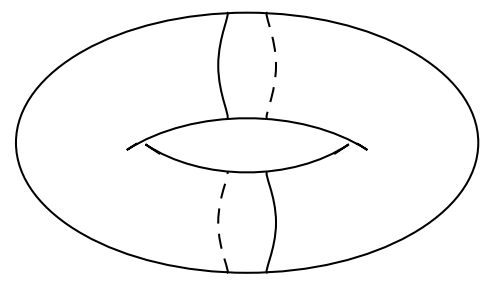

FIGURA 3.4: Toro

\subsection{Funções meromorfas}

Antes de partir para as próximas definições, faz-se necessário recordar três conceitos fundamentais.

1. Polo é uma singularidade do tipo $\frac{1}{z^{n}}$ em $z=0$.

2. Funções holomorfas são funções definidas sobre abertos $U \subset \mathbb{C}$ com valores em $\mathbb{C}$ que são diferenciáveis em cada ponto $z_{0} \in U$.

3. Funções meromorfas são definidas sobre abertos $U \subset \mathbb{C}$ com valores em $\mathbb{C}$ que são holomorfas em todo $U$, exceto por um conjunto de pontos isolados, que são os polos da função.

Por definição uma variedade complexa $\Sigma$ admite uma cobertura por cartas coordenadas $\left(U_{i}, z_{i}\right)_{i \in J}$. As coordenadas $z_{i}$ estão relacionadas a mudanças de coordenadas holomorfas $z_{j}=z_{j}\left(z_{i}\right)$. No contexto deste trabalho, é importante compreender o que são funções holomorfas numa superfície de Riemann. Funções holomorfas podem ser definidas localmente como funções holomorfas quando aplicadas nas coordenadas $z_{i}$. Esta noção pode ser estendida para obtermos funções meromorfas.

\section{4 Álgebras correntes}

Para as construções a seguir, serão importantes dados geométricos preestabelecidos. O par $(\Sigma, A)$ denota uma superfície de Riemann $\Sigma$ enquanto que $A$ denota um subconjunto de pontos em $\Sigma$ que serão utilizados para construir uma álgebra de funções meromorfas em $\Sigma$, holomorfas fora de $A \subset \Sigma$ que será denotada por $\mathcal{A}$ e é comumente chamada de álgebra de funções.

A álgebra de funções tem uma base $\left\{A_{n}=z^{n} \mid n \in \mathbb{Z}\right\}$. Note que

$$
\left[A_{m}, A_{n}\right]=A_{m+n}
$$

o que nos mostra que $\mathcal{A}$ é graduada.

Exemplo 3.4.1 Considere a dupla $\left(S^{2}, A\right)$ em que $A=\{0, \infty\}$. A álgebra $\mathcal{A}$ é formada pelos polinômios de Laurent definidos sobre a esfera de Riemann, isto é, $\mathcal{A}=\mathbb{C}\left[t, t^{-1}\right]$.

Diremos que as álgebras obtidas a partir destes dados geométricos pré estabelecidos tem gênero $g=g(\Sigma)$. Como notamos no Exemplo 3.2.1, a esfera de Riemann tem gênero 0 e por consequência a álgebra dos polinômios de Laurent definida no Exemplo 3.4.1 tem gênero 0.

Considere $\mathfrak{g}$ uma álgebra de Lie de dimensão finita. A álgebra corrente associada à $\mathfrak{g}$ é dada por

$$
\overline{\mathfrak{g}}=\mathfrak{g} \otimes \mathcal{A}=\mathfrak{g} \otimes \mathbb{C}\left[t, t^{-1}\right] .
$$


Os elementos de $\overline{\mathfrak{g}}$ podem ser descritos como somas de $x \otimes t^{n} \operatorname{com} x \in \mathfrak{g}$. Quando $\mathfrak{g}$ é uma álgebra de matrizes, $\overline{\mathfrak{g}}$ pode ser descrita como uma álgebra de matrizes cujas entradas são polinômios de Laurent. A estrutura de Lie é dada pela seguinte relação:

$$
\left[x \otimes t^{m}, y \otimes t^{n}\right]=[x, y] \otimes t^{m+n}
$$

para todos $x, y \in \mathfrak{g}, t^{m}, t^{n} \in \overline{\mathfrak{g}}$ e $m, n \in \mathbb{Z}$. A álgebra $\overline{\mathfrak{g}}$ herda uma graduação da graduação de $\mathcal{A}$.

Generalizaremos a construção de uma álgebra de Lie corrente para uma de gênero maior.

Definição 3.4.2 Uma álgebra corrente de gênero maior (também chamada de álgebra multiponto, ou álgebra $N$-ponto, em que $N=\# A$ ) associada a uma álgebra de Lie $\mathfrak{g} e$ aos dados geométricos $(\Sigma, A)$ é a álgebra de Lie $\overline{\mathfrak{g}}=\overline{\mathfrak{g}}(A)=\overline{\mathfrak{g}}(\Sigma, A)$ dada, como espaço vetorial pela álgebra tensor $\overline{\mathfrak{g}}=\mathfrak{g} \otimes_{\mathrm{C}} \mathcal{A}$ com o seguinte produto de Lie

$$
[x \otimes f, y \otimes g]=[x, y] \otimes f \cdot g
$$

para todos $x, y \in \mathfrak{g} e f, g \in \mathcal{A}$.

É fácil verificar que $\overline{\mathfrak{g}}$ é uma álgebra de Lie. Os elementos de $\overline{\mathfrak{g}}$ podem ser interpretados como funções meromorfas de $\Sigma$ em $\mathfrak{g}$ que são holomorfas fora de $A$. $\mathcal{A}$.

Podemos replicar esta construção para qualquer álgebra comutativa e associativa

Lembre-se de que se a álgebra $\mathfrak{g}$ é tal que $[\mathfrak{g}, \mathfrak{g}]=\mathfrak{g}$, então dizemos que $\mathfrak{g}$ é perfeita (veja a Seção 2.1.3).

Proposição 3.4.3 ([Sch14b], Proposição 9.2) Se gé uma álgebra de Lie perfeita de dimensão finita, então a álgebra corrente $\mathfrak{\mathfrak { g }}$ também é perfeita. Em particular, se gé semisimples $\overline{\mathfrak{g}}$ é perfeita.

Quando não for necessário mencionar, os dados geométricos serão suprimidos.

\subsection{A situação de Krichever-Novikov}

\subsubsection{O caso 1-ponto}

A escolha natural ao considerar uma generalização de uma álgebra de Lie corrente é tomar $A$ consistindo de um único ponto em $\Sigma$, digamos $A=\{P\}$. Teremos que $\Sigma \backslash\{P\}$ é uma superfície de Riemann aberta (isto é, uma superfície de Riemann sem fronteira). Para esta situação, não é possível aplicar a noção usual de graduação nas álgebras obtidas. Discutiremos como lidar com estruturas que não admitem tal graduação usual na Seção 3.6 na qual será apresentada a "quasegraduação". Entretanto, são necessários ao menos dois pontos em $A$ para fixarmos uma quasegraduação. $\mathrm{Na}$ Definição 3.5.2, impomos que do caso 1-ponto não é possível compor uma álgebra do tipo Krichever-Novikov.

\subsubsection{A situação de Krichever-Novikov generalizada}

No trabalho de Krichever e Novikov ( [KN87] ), originalmente é considerado o caso em que $A$ possui 2 pontos. Conforme já mencionado, no decorrer deste trabalho serão apresentadas outras situações.

Dividiremos o conjunto $A$ em dois subconjuntos: $I$, os pontos de entrada e $O$ pontos de saída, de forma que $A=I \cup O$. Esta divisão será utilizada para construirmos a quasegraduação.

A seguir, veja outro exemplo de álgebra em que $\# A=2$. 
Exemplo 3.5.1 Considere a álgebra $\mathcal{L}$ que possui base dada por $e_{n}=z^{n+1} \frac{d}{d z}, n \in \mathbb{Z} e$ equações de estrutura

$$
\left[e_{n}, e_{m}\right]=(m-n) e_{n+m} .
$$

Esta é a álgebra formada por campos de vetores na esfera de Riemann holomorfos fora de $0 e$ $\infty$ e é conhecida como álgebra de Witt ou álgebra de Virasoro sem elemento central.

Conforme mencionado no início deste capítulo, as álgebras de Krichever-Novikov são exemplos de álgebras de Lie de dimensão infinita cujos componentes são objetos meromorfos numa superfície de Riemann compacta que são holomorfos fora de um conjunto finito de pontos. Consideraremos a seguinte definição para álgebras de Krichever-Novikov.

Definição 3.5.2 Dada uma superfície de Riemann $\Sigma$ e um conjunto finito de pontos $A$ em $\Sigma$, dividido em dois subconjuntos de pontos I e $O$, em que $A=I \cup O, \# A \geq 2$, então as álgebras do tipo $\mathcal{A}$ e $\overline{\mathfrak{g}}$ são álgebras de Krichever-Novikov.

Em [Sch14b] é possível obter uma definição mais generalizada de álgebras de Krichever-Novikov, bem como exemplos mais amplos. No entanto, a Definição 3.5.2 cobre todos os casos que serão trabalhados neste e nos próximos capítulos, sem a necessidade de uma investigação ainda mais profunda em aspectos geométricos. É importante registrar que o que deve ser chamado de uma álgebra do tipo KricheverNovikov, não é a apenas a álgebra, mas a álgebra junto com uma quasegraduação escolhida. Por essa razão e para que fique preciso do que se trata uma álgebra do tipo Krichever-Novikov, será esclarecido na próxima seção o que é uma quasegraduação.

\subsection{Quasegraduação}

Além de continuar a denotar por $\Sigma$ uma superfície de Riemann compacta de gênero $g=g(\Sigma)$ e por $A$ um subconjunto finito de $\Sigma$, indicaremos por $N$ a cardinalidade do conjunto $A$, isto é, $N:=\# A$. Assumiremos que $N \geq 2$ a menos de menção contrária e continuaremos a dividir o conjunto $A$ em dois subconjuntos disjuntos de forma que $A=I \cup O$, da mesma forma que foi apresentada na Subseção 3.5. Por último, consideraremos que

$$
K:=\# I, M:=\# O \text { e, por definição, } N=K+M .
$$

Descreveremos os conjuntos $I$ e $O$ desta forma:

$$
I=\left(P_{1}, \ldots, P_{K}\right) \text { e } O=\left(Q_{1}, \ldots, Q_{M}\right)
$$

as n-uplas de pontos disjuntos (que podem ser chamados de pontuações ou pontos marcados) na superfície de Riemann. Assumiremos que $P_{i} \neq Q_{j}$ para todo par $(i, j)$. Conforme já mencionado, os pontos em $I$ serão chamados de pontos de entrada e os pontos em $O$ serão chamados de pontos de saída e a decomposição de $A$ em pontos de entrada e saída é chamada de cisão de $A$.

Segundo [Sch14b], há uma interpretação para esta cisão em Teoria de Cordas: $\Sigma$ corresponde à folha de Universo, o conjunto $I$ corresponde às cordas livres e os pontos em $O$ correspondem às cordas livres de saída. Este tipo de interpretação aqui tem o objetivo de registrar para o leitor o vínculo forte que a teoria algébrica desenvolvida aqui tem com a Física Matemática. 
Daqui em diante, quando mencionada a expressão situação clássica, estaremos nos referindo à seguinte configuração:

$$
\Sigma=S^{2} \text {, em que } I=\{0\} \text { e } O=\{\infty\} .
$$

Na situação clássica, em situações com gêneros maiores, ou quando considerando mais que dois pontos marcados, não é possível obter uma graduação das álgebras obtidas que não seja a trivial. Defronte à esta questão, Krichever e Novikov em [KN87] introduziram um conceito mais fraco do que a graduação nomeado quasigraduation. Este conceito foi estendido por outros autores considerando casos com mais que dois pontos marcados (veja, por exemplo, [Sch90a]). Este tipo de graduação será construído levando em consideração a cisão de $A$ e, neste trabalho, esta expressão é traduzida para quasegraduação.

Definição 3.6.1 1. Seja $\mathcal{L}$ uma álgebra de Lie. Diremos que $\mathcal{L}$ é uma álgebra de Lie quasegraduada $s e$

(a) L é um espaço vetorial soma direta

$$
\mathcal{L}=\bigoplus_{n \in \mathbb{Z}} \mathcal{L}_{n}
$$

(b) $\operatorname{dim} \mathcal{L}_{n}<\infty$

(c) Existem constantes $L_{1}$ e $L_{2}$ tais que

$$
\left[\mathcal{L}_{n}, \mathcal{L}_{m}\right] \subseteq \bigoplus_{h=n+m-L_{1}}^{n+m+L_{2}} \mathcal{L}_{h} .
$$

Os elementos $\mathcal{L}_{n}$ são chamados de elementos homogêneos de grau n e $\mathcal{L}_{n}$ é chamado de subespaço homogêneo de grau n.

2. Se $\mathcal{L}$ é uma álgebra graduada e $\mathcal{M}$ é um $\mathcal{L}$-módulo, dizemos que $\mathcal{M}$ é um módulo quasegraduado se

(a) $\mathcal{M}$ é um espaço vetorial soma direta

$$
\mathcal{M}=\bigoplus_{n \in \mathbb{Z}} \mathcal{M}_{n}
$$

(b) $\operatorname{dim} \mathcal{M}_{n}<\infty$

(c) Existem constantes $M_{1}, M_{2} \in \mathbb{Z}$ tais que

$$
\mathcal{L}_{n} \cdot \mathcal{M}_{m} \subseteq \bigoplus_{h=n+m-M_{1}}^{n+m+M_{2}} \mathcal{M}_{h}
$$

Os elementos $\mathcal{M}_{n}$ são chamados de elementos homogêneos de grau $n$ e $\mathcal{M}_{n}$ é chamado de subespaço homogêneo de grau n.

3. Quando nem todos subespaços homogêneos são de dimensão finita, a estrutura é chamada de fracamente quasegraduada.

4. Quando as dimensões são limitadas, independente de n, a estrutura é chamada de fortemente quasegraduada. 
Para efeito de comparação com a definição de graduação, observe a Definição 2.1.13 e a Definição 2.2.4.

Proposição 3.6.2 ([Sch14b], Proposição 3.12) As álgebras de Krichever-Novikov do tipo $\mathcal{A}$ são fortemente quasegraduadas.

Veja a seguir um exemplo de álgebra fortemente quasegraduada.

Exemplo 3.6.3 Seja $\overline{\mathfrak{g}}=\mathfrak{g} \otimes \mathcal{A}$ a álgebra corrente do tipo Krichever-Novikov associada à álgebra de Lie de dimensão finita $\mathfrak{g}$. Considere $\mathcal{A}_{n}$ subconjunto de $\mathcal{A}$ formado por elementos $f$ de grau $\operatorname{deg} f=n$, por exemplo

$$
t^{-2}+5 t^{7} \in \mathcal{A}_{7}, \text { pois } \operatorname{deg}\left(t^{-2}+5 t^{7}\right)=7 .
$$

Admita que a dimensão de $\mathcal{A}_{n}$ é K e que o gênero de $\mathcal{A}_{n}$ é $R_{1}$. Definindo

$$
\overline{\mathfrak{g}_{n}}:=\mathfrak{g} \otimes \mathcal{A}_{n},
$$

obteremos que

$$
\overline{\mathfrak{g}}:=\bigoplus_{n \in \mathbb{Z}} \mathfrak{g}_{n}, \quad \operatorname{dim} \overline{\mathfrak{g}_{n}}=K \cdot \operatorname{dim} \mathfrak{g}
$$

$e$

$$
\left[\overline{\mathfrak{g}_{n}}, \overline{\mathfrak{g}_{m}}\right] \subseteq \bigoplus_{h=n+m}^{n+m+R_{1}}
$$

Portanto, $\overline{\mathfrak{g}}$ é uma álgebra fortemente quasegraduada.

Podemos construir uma quasegraduação de $\mathcal{A}=\oplus_{n \in \mathbb{Z}}(\mathcal{A})_{n}$ induzida por determinada cisão, induzindo uma quasegraduação

$$
\overline{\mathfrak{g}}=\bigoplus_{n \in \mathbb{Z}}(\overline{\mathfrak{g}})_{n}
$$

em que $\overline{\mathfrak{g}}_{n}=\mathfrak{g} \otimes \mathcal{A}_{n}$, via $\operatorname{deg}\left(\overline{\mathfrak{g}}_{n}\right):=n$. A quase-graduação depende crucialmente da cisão de $A$ em $I \cup O$. De fato, cisões diferentes, podem fornecer quase-graduações diferentes. Para detalhes técnicos relacionados à quase-graduações, veja [Sch14b].

\subsection{Extensões centrais universais de álgebras de Krichever- Novikov}

Segundo [Sch14b], extensões centrais aparecem naturalmente no contexto de quantizações da Teoria de Campos Clássica. Nesta seção é apresentada uma definição destas estruturas algébricas.

\subsubsection{Complexos e cohomologia}

Definição 3.7.1 Uma sequência de objetos $C_{i}$ com $i \in \mathbb{Z}$, de uma categoria abeliana $\mathcal{C} e$ morfismos $d_{i}: C_{i} \rightarrow C_{i+1}$ é chamada um complexo se a composição de dois morfismos consecutivos é zero. Os morfismos consecutivos são chamados de diferenciais.

Exemplo 3.7.2 O complexo de de Rham ${ }^{3}$ é obtido considerando que $C_{m}$ é o espaço das mformas diferenciais. Esta é a razão para a utilização do termo "diferenciais", na definição anterior.

\footnotetext{
${ }^{3}$ Georges de Rham (1903 - 1990) foi um matemático suíço, conhecido por seu trabalho sobre topologia diferencial.
} 
A cohomologia de um complexo é $H^{i}=\operatorname{Ker}\left(d_{i}\right) / \operatorname{Im}\left(d_{i-1}\right)$

Podemos considerar complexos sobre estruturas como grupos abelianos, espaços vetoriais ou módulos sobre um anel. Nesses casos, os elementos $\operatorname{Ker}\left(d_{i}\right)$ são chamados $i$-cociclos, os elementos de $\operatorname{Im}\left(d_{i-1}\right)$ são chamados de $i$-colimites e os elementos em $H^{i}(C)$ são chamados de $i$-ésimas classes de cohomologia.

Exemplo 3.7.3 Uma sequência exata curta é um complexo da seguinte forma

$$
0 \longrightarrow X \longrightarrow Y \longrightarrow Z \longrightarrow 0 \text {. }
$$

em que $X \longrightarrow Y$ deve ser injetor, $Y \longrightarrow Z$ deve ser sobrejetor e $Y / X \rightarrow Z$ deve ser $u m$ isomorfismo.

Podemos interpretar a sequência exata curta (3.1) como uma extensão $Y$ de $Z$ por $X$.

\subsubsection{Extensões centrais}

Seja $L$ uma álgebra de Lie. Uma extensão central (unidimensional) $\widehat{L}$ de $L$ é o elemento do meio na sequência exata curta de álgebras de Lie

$$
0 \longrightarrow \mathbb{C} \stackrel{i}{\rightarrow} \widehat{L} \stackrel{p}{\rightarrow} L \longrightarrow 0
$$

em que $i(\mathbb{C})$ é central em $\widehat{L}$.

Duas extensões centrais são $\widehat{L_{1}}$ e $\widehat{L_{2}}$ são equivalentes se existe um isomorfismo de algebras de Lie $\varphi: \widehat{L_{1}} \rightarrow \widehat{L_{2}}$ tal que o diagrama

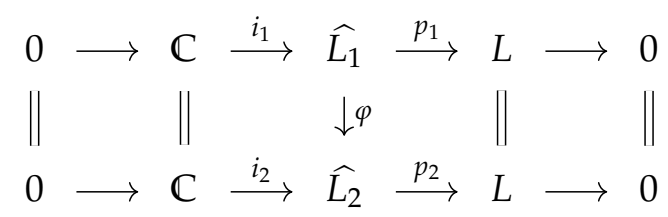

comuta.

Em (3.2), sempre é possível encontrar $s: L \rightarrow \widehat{L}$ que cinde a sequência, isto é $p \circ s=i d_{L}$.

Definição 3.7.4 Uma extensão central $\widehat{L}$ de $L$

$$
0 \longrightarrow K \stackrel{i}{\rightarrow} \widehat{L} \stackrel{p}{\rightarrow} L \longrightarrow 0
$$

é dita uma extensão central universal se para toda extensão central $\widehat{L}^{\prime}$ de L (com termo central $K^{\prime}$ )

$$
0 \longrightarrow K^{\prime} \stackrel{i^{\prime}}{\rightarrow} \widehat{L}^{\prime} \stackrel{p}{\rightarrow} L \longrightarrow 0
$$

existe um único par de homomorfismos de Lie $\left(\phi, \phi_{0}\right):(K, \widehat{L}) \mapsto\left(K^{\prime}, \widehat{L}^{\prime}\right)$ tal que o diagrama

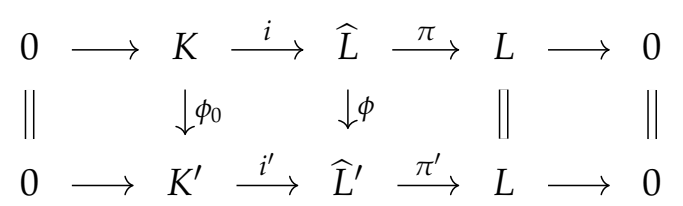

comuta.

Teorema 3.7.5 ([Sch14b], Teorema 6.98) Toda álgebra de Lie perfeita admite extensão central universal. 
Para encerrar esta seção, enunciaremos um teorema fundamental para o principal resultado deste capítulo. Trata-se de um teorema de Kassel, publicado em [KL82] e que oferece uma descrição da extensão central universal do tipo de álgebras de Lie em que estamos interessados nestas seções.

Teorema 3.7.6 ([KL82]) Considere $\mathfrak{g}$ uma álgebra de Lie complexa, simples e de dimensão finita e $R$ uma álgebra complexa, comutativa e associativa. Temos que $\hat{\mathfrak{g}}=(\mathfrak{g} \otimes R) \oplus$ $\Omega_{R}^{1} / d R$ é a extensão central universal de $\mathfrak{g} \otimes R$, em que $\Omega_{R}^{1} / d R$ é o espaço de diferenciais de Kähler de $R$ módulo formas exatas.

Para compreender o Teorema 3.7.6 é necessário retomar a definição do espaço dos diferenciais de Kähler ${ }^{4}$. É possível construí-lo de forma relativamente simples.

Definição 3.7.7 Seja K o submódulo do módulo $R \otimes R$ gerado por elementos $1 \otimes f g-f \otimes$ $g-g \otimes f$, em que $f, g \in R$. Considere $F=R \otimes R$ um $R$-módulo à esquerda com ação $f(g \otimes h)=f g \otimes h$ em que $f, g, h \in R$. O módulo quociente $\Omega_{R}^{1}=F / K$ é o módulo de diferenciais de Kähler de $R$.

Denotaremos o elemento $f \otimes g+K$ de $\Omega_{R}^{1}$ por $f d g$. Definiremos uma aplicação $d$ : $R \rightarrow \Omega_{R}^{1}$ por $d(f)=1 \otimes f+K$. Os elementos no subespaço $d R=d(R)$ são chamados diferenciais exatos. Denotaremos a coclasse $f d g$ módulo $d R$ por $\overline{f d g}$. As relações de comutatividade para $(\mathfrak{g} \otimes R) \oplus \Omega_{R}^{1} / d R$ são

$$
\begin{aligned}
{[x \otimes f, y \otimes g] } & =[x, y] \otimes f g+(x, y) \overline{f d g}, \\
{[x \otimes f, \omega] } & =0,
\end{aligned}
$$

em que $x, y \in \mathfrak{g}, f, g \in R$ e $\omega \in \Omega_{R}^{1} / d R$ e $(x, y)$ denota a forma de Cartan-Killing em $\mathfrak{g}$, conforme definida em (2.4) (daqui em diante representada omitindo $\beta$ ).

\subsection{3 Álgebras multiponto de gênero zero}

\section{O caso 4-ponto}

Considere $\mathfrak{g}$ uma álgebra de Lie complexa de dimensão finita e simples. Seja $P_{4}=$ $\left\{a_{1}, a_{2}, a_{3}, a_{4}\right\}$ o conjunto formado por quatro pontos distintos na esfera de Riemann. $\mathrm{O}$ anel 4-ponto $R_{4}$ é o anel das funções racionais na esfera de Riemann com polos permitidos somente no conjunto $P_{4}$.

Definição 3.7.8 Escrevendo $a_{4}=a$, o anel 4-ponto $R_{4}=\mathbb{C}\left[s, s^{-1},(s-1)^{-1}\right.$, $\left.(s-a)^{-1} \mid a \in \mathbb{C} \backslash\{0,1\}\right]$ é o anel das funções racionais na esfera de Riemann com polos permitidos somente em $\left\{a_{1}, a_{2}, a_{3}, a_{4}\right\}=\{\infty, 0,1, a\}$. Similarmente, o anel das funções racionais na esfera de Riemann com polos permitidos somente em um conjunto de $N$ pontos será chamado anel $N$-ponto e será denotado por $R_{N}$.

Proposição 3.7.9 ([Bre95], Proposição 1.1) Considere $S_{b}$ o anel $\mathbb{C}\left[t, t^{-1}, u\right]$ em que $u^{2}=$ $t^{2}-2 b t+2$. Temos que $R_{4} \cong S_{b}$, escolhendo $a=(b+1) /(b-1) \in \mathbb{C} \backslash\{0,1\}$ ou $b=(a+1) /(a-1) \in \mathbb{C} \backslash\{-1,1\}$.

A proposição anterior nos assegura que $R_{4}$ é um anel isomorfo a um anel do tipo $\mathbb{C}\left[t, t^{-1}, u\right] /\left\langle u^{m}-p(t)\right\rangle$, em que $m \in \mathbb{N}$ e $p(t) \in \mathbb{C}[t]$.

\footnotetext{
${ }^{4}$ Erich Kähler (1906 - 2000) foi um matemático alemão com contribuições significativas em geometria.
} 
Definição 3.7.10 O espaço vetorial $\mathcal{A}_{4}:=\mathfrak{g} \otimes R_{4}$ munido das relações de comutatividade

$$
[x \otimes f, y \otimes g]=[x, y] \otimes f g
$$

em que $x, y, \in \mathfrak{g} e f, g \in R_{4}$ é uma álgebra de Lie denominada álgebra laço 4-ponto.

A partir daqui consideraremos

$$
\omega_{0}:=\overline{t^{-1} d t}, \quad \omega_{-}:=\overline{t^{-2} u d t}, \quad \text { e } \quad \omega_{+}:=\overline{t^{-1} u d t}
$$

Definição 3.7.11 A extensão central universal de $\mathcal{A}_{4}$, a álgebra de Lie definida por

$$
\widehat{\mathcal{A}_{4}}:=\left(\mathfrak{g} \otimes R_{4}\right) \oplus \Omega_{R_{4}}^{1} / d R_{4},
$$

é chamada de álgebra afim 4-ponto.

Dada uma álgebra de Lie complexa de dimensão finita $\mathfrak{g} e R_{N}$ um anel $N$-ponto, vamos denominar como álgebra afim N-ponto a álgebra de Lie dada por $\left(\mathfrak{g} \otimes R_{N}\right) \oplus \Omega_{R_{N}}^{1} / d R_{N}$. É possível determinar uma base finita para o centro de $\widehat{\mathcal{A}_{4}}$.

Teorema 3.7.12 ([Bre95], Teorema 3.3) O espaço $\Omega_{R_{4}}^{1} / d R_{4}$ tem base $\left\{\omega_{0}, \omega_{-}, \omega_{+}\right\}$.

Além disso, é possível descrever as relações satisfeitas pelos elementos da base de $\widehat{\mathcal{A}_{4}}$, que são $x \otimes t^{n}, x \otimes t^{n} u, \omega_{0}$, e $\omega_{ \pm}$. Para apresentar tais relações, é necessário definir uma família de polinômios $Q_{k}(b)$ dados em termos dos polinômios ultraesféricos de Gegenbauer $P_{k}^{\lambda}$ (veja o Exemplo 4.1.2 para retomar polinômios ultraesféricos de Gegenbauer). Consideremos $P_{k}(b)=P_{k}^{-1 / 2}(b)$ e definamos para $b \neq \pm 1$,

$$
Q_{k}(b):=-\frac{P_{k}+2(b)}{b^{2}-1}
$$

em que $k \in \mathbb{N} \cup\{0\}$.

Teorema 3.7.13 ([Bre95], Teorema 3.6) Aálgebra afim 4-ponto $\widehat{\mathcal{A}_{4}}$ tem uma $\mathbb{Z} / 2 \mathbb{Z}$-graduação em que

$$
{\widehat{\mathcal{A}_{4}}}^{0}=\mathfrak{g} \otimes \mathbb{C}\left[t, t^{-1}\right] \oplus \mathbb{C} \omega_{0} \quad e \quad{\widehat{\mathcal{A}_{4}}}^{1}=\mathfrak{g} \otimes \mathbb{C}\left[t, t^{-1}\right] u \oplus \mathbb{C} \omega_{-} \oplus \mathbb{C} \omega_{+} .
$$

Um conjunto gerador de $\widehat{\mathcal{A}_{4}}$ consiste dos elementos $x \otimes t^{i}$ e $x \otimes t^{j-\frac{1}{2}} u$ em que $x \in \mathfrak{g}, i \in \mathbb{Z}$, $j \in \mathbb{Z}+\frac{1}{2}$, com os elementos centrais $\omega_{0}, \omega_{+}$, e $\omega_{-}$.

A subálgebra par ${\widehat{\mathcal{A}_{4}}}^{0}$ é uma álgebra de Lie afim de Kac-Moody não torcida com as seguintes relações de comutatividade.

$$
\left[x \otimes t^{i}, y \otimes t^{j}\right]=[x, y] \otimes t^{i+j}+\delta_{i+j, 0}(x, y) j \omega_{0} .
$$

As relações de comutatividade dos dois elementos de ${\widehat{\mathcal{A}_{4}}}^{1}$ resultam em elementos em $\widehat{\mathcal{A}}_{4}^{0}$ :

$$
\begin{aligned}
{\left[x \otimes t^{i-\frac{1}{2}} u, y \otimes t^{j-\frac{1}{2}} u\right] } & =[x, y] \otimes\left(t^{i+j-1}-2 b t^{i+j}+t^{i+j+1}\right) \\
& +(x, y)\left\{\begin{array}{l}
-2 j b \omega_{0,}, \text { se } i+j=0, \\
\frac{1}{2}(j-i) \omega_{0},, s e|i+j|=1, \\
0,, s e|i+j| \geq 2 .
\end{array}\right.
\end{aligned}
$$

O subespaço impar $\widehat{\mathcal{A}}_{4}^{1}$ é um ${\widehat{\mathcal{A}_{4}}}^{0}$-módulo com relações dadas por

$$
\begin{aligned}
{\left[x \otimes t^{i-\frac{1}{2}} u, y \otimes t^{j}\right] } & =[x, y] \otimes\left(t^{i+j-\frac{1}{2}} u\right) \\
+(x, y) & \left\{\begin{array}{l}
j Q_{i+j-\frac{3}{2}}(b)\left(b \omega_{+}+\omega_{-}\right) s e i+j \geq \frac{3}{2} ; \\
j \omega_{ \pm} s e i+j= \pm \frac{1}{2} ; \\
j Q_{-i-j-\frac{3}{2}}(b)\left(b \omega_{+}+b \omega_{-}\right) s e i+j \leq-\frac{3}{2} .
\end{array}\right.
\end{aligned}
$$




\section{O caso 3-ponto}

Nesta subseção, considere $\mathfrak{g}=\mathfrak{s l}(2, \mathbb{C})$. Seja $P_{3}=\left\{a_{1}, a_{2}, a_{3}\right\}$ o conjunto formado por três pontos distintos na esfera de Riemann. O anel 3-ponto é o anel das funções racionais na esfera de Riemann com polos permitidos somente no conjunto $P_{3}$.

Os anéis 3-ponto se apresentam de, pelo menos, três formas:

$$
\begin{aligned}
& S_{3}:=\mathbb{C}\left[s, s^{-1},(s-1)^{-1}\right] \\
& R_{3}:=\mathbb{C}\left[t, t^{-1}, u\right]\left\langle u^{2}-\left(t^{2}+4 t\right)\right\rangle \mathrm{e} \\
& A_{3}:=\mathcal{A}_{a}=\mathbb{C}\left[\left(z^{2}-a^{2}\right)^{k}, z\left(z^{2}-a^{2}\right)^{k} \mid k \in \mathbb{Z}\right]
\end{aligned}
$$

É possível verificar que há um isomorfismo entre esses três anéis (veja [CJ14]).

Teorema 3.7.14 ([CJ14], Proposição 2.2) Uma base para $\Omega_{R_{3}}^{1} / d R_{3} e ́\left\{\omega_{0}:=\overline{t^{-1} d t}, \omega_{1}:=\right.$ $\left.\overline{t^{-1} u d t}\right\}$.

Denotaremos a álgebra laço 3-ponto $\mathfrak{g} \otimes R_{3}$ por $\mathcal{A}_{3}$ e a álgebra afim 3-ponto, a extensão central universal de $\mathcal{A}_{3}$, por $\widehat{\mathcal{A}_{3}}$.

Teorema 3.7.15 ([CJ14], Teorema 2.4) A álgebra afim 3-ponto $\widehat{\mathcal{A}_{3}}$ é isomorfa à álgebra dada por geradores e relações abaixo. Considere os geradores $e_{n}, e_{n}^{1}, f_{n}, f_{n}^{1}, h_{n}, h_{n}^{1}, n \in \mathbb{Z}, w_{0}, w_{1}$ e relações dadas por

$$
\begin{aligned}
& {\left[x_{m}, x_{n}\right] }:=\left[x_{m}, x_{n}^{1}\right]=\left[x_{m}^{1}, x_{n}^{1}\right]=0, \text { para } x=e, f, \\
& {\left[h_{m}, h_{n}\right] }:=-2 m \delta_{m,-n} \omega_{0}=(n-m) \delta_{m,-n} \omega_{0}, \\
& {\left[h_{m}^{1}, h_{n}^{1}\right] }:=2\left((n+1) \delta_{m+n,-2}+(4 n+2) \delta_{m+n,-1}\right) \omega_{0}=(n-m)\left(\delta_{m+n,-2}+4 \delta_{m+n,-1}\right) \omega_{0}, \\
& {\left[h_{m}, h_{n}^{1}\right]:=-2 m \delta_{m, n} \omega_{1} } \\
& {\left[\omega_{i}, x_{m}\right]:=\left[\omega_{i}, \omega_{j}\right]=0 \text { para } x=e, f, h e i, j \in\{0,1\} } \\
& {\left[e_{m}, f_{n}\right]:=h_{m+n}-m \delta_{m,-n} \omega_{0}, } \\
& {\left[e_{m}, f_{n}^{1}\right]:=h_{m+n}^{1}-m \delta_{m,-n} \omega_{1}=:\left[e_{m}^{1}, f_{n}\right] } \\
& {\left[e_{m}^{1}, f_{n}^{1}\right]:=h_{m+n+2}+4 h_{m+n+1}+\left((n+1) \delta_{m+n,-2}+(4 n+2) \delta_{m+n,-1}\right) \omega_{0} } \\
& {\left[h_{m}, e_{n}\right]:=2 e_{m+n}, } \\
& {\left[h_{m}, e_{n}^{1}\right]:=2 e_{m+n}^{1}=:\left[h_{n}^{1}, e_{m}\right], } \\
& {\left[h_{m}^{1}, e_{n}^{1}\right]:=2 e_{m+n+2}+8 e_{m+n+1}, } \\
& {\left[h_{m}, f_{n}\right]:=-2 f_{m+n,} } \\
& {\left[h_{m}, f_{n}^{1}\right]:=-2 f_{m+n}^{1}=:\left[h_{n}^{1}, f_{m}\right], } \\
& {\left[h_{m}^{1}, f_{n}^{1}\right]:=-2 f_{m+n+2}-8 f_{m+n+1}, }
\end{aligned}
$$

para todo $m, n \in \mathbb{Z}$.

\subsection{4 Álgebras de gênero maior ou igual a zero}

\section{O caso hiperelíptico}

As álgebras de Lie laço hiperelípticas formam uma família de álgebras de KricheverNovikov. Os resultados que serão apresentados nesta subseção têm papel fundamental para o desenvolvimento da subseção 3.8, pois alguns dos resultados apresentados aqui são lá estendidos. 
Para construir as álgebras de Lie laço hiperelípticas, é necessário definir o anel hiperelíptico.

Definição 3.7.16 $O$ anel hiperelíptico $R_{H}$ é o anel formado pelas funções hiperelípticas, isto é, $R_{H}=\mathbb{C}\left[t, t^{-1}, u\right]$ em que $u^{2}=k(t) \in \mathbb{C}[t]$. A equação $u^{2}=p(t)$ define uma curva hiperelíptica.

Definição 3.7.17 O espaço vetorial $\mathcal{A}_{H}:=\mathfrak{g} \otimes R_{H}$, munido das relações de comutatividade

$$
[x \otimes f, y \otimes g]=[x, y] \otimes f g
$$

em que $x, y \in \mathfrak{g}$ e $f, g \in R_{H}$ é uma álgebra de Lie denominada álgebra laço hiperelíptica

Definição 3.7.18 A extensão central universal de $\mathcal{A}_{H}$, a álgebra de Lie definida por

$$
\widehat{\mathcal{A}_{H}}:=\left(\mathfrak{g} \otimes R_{H}\right) \oplus \Omega_{R_{H}}^{1} / d R_{H},
$$

é chamada de álgebra afim hiperelíptica.

A dimensão e a descrição explícita da base para o centro de $\widehat{\mathcal{A}_{H}}$ é conhecida, considerando o caso em que $p(t)=\sum_{i=0}^{D} a_{i} t^{i} \in \mathbb{C}\left[t, t^{-1}\right]$ de tal forma que $a_{D}=1$ e $a_{0}, a_{1}$ não são ambos nulos.

Teorema 3.7.19 ([Bre94], Teorema 2.1) A dimensão do centro $\Omega_{R_{H}}^{1} / d R_{H}$ da extensão central universal de $\mathfrak{g} \otimes R_{H}$ é $2 g+n-1$, em que g é o gênero da curva algébrica dada por $u^{2}=k(t)$ vista como uma superfície de Riemann com $n$ pontos distintos removidos.

Teorema 3.7.20 ([Bre94], Teorema 3.4) Uma base para $\Omega_{R}^{1} / d R$ é dada por $\overline{t^{-1} d t} e$ $\overline{t^{-1} u d t} \ldots, \overline{t^{-D} u d t}$, em que omitimos $\overline{t^{-D} u d t}$ se $a_{0}=0$.

\section{O caso elíptico}

Seja $\Sigma$ uma curva algébrica complexa compacta não singular de gênero 1 . Denotando por $\Sigma$ o quociente do plano complexo $\mathbb{C}$ pelo reticulado $\Lambda=\mathbb{Z} \oplus \mathbb{Z} \lambda$ com base $\{1, \lambda\}$ em que $\operatorname{Im} \lambda>0^{5}$. Por conveniência identificaremos um elemento $z \in \mathbb{C}$ e o ponto correspondente $z+\Lambda$.

Definição 3.7.21 O anel elíptico $R_{E}$ é o anel formado por todas as funções meromorfas $\Sigma$, holomorfas fora do conjunto $\{0, \mu\}$ em que $\mu=\frac{1}{2}(1+\lambda)$

Proposição 3.7.22 ([Bre94], Proposição 4.1) Se $b=-6 m /\left(12 m^{2}-60 \sum_{\xi \in \Lambda \backslash\{0\}} \xi^{-4}\right)^{2}$, então $R \cong \mathbb{C}\left[t, t^{-1}, u\right] /\left\langle u^{2}-\left(t^{3}-2 b t^{2}+t\right)\right\rangle$.

Definição 3.7.23 O espaço vetorial $\mathcal{A}_{E}:=\mathfrak{g} \otimes R_{E}$, munido das relações de comutatividade

$$
[x \otimes f, y \otimes g]=[x, y] \otimes f g
$$

em que $x, y \in \mathfrak{g}$ e $f, g \in R_{E}$ é uma álgebra de Lie denominada álgebra laço elíptica.

Definição 3.7.24 A extensão central universal de $\mathcal{A}_{E}$, a álgebra de Lie definida por

$$
\widehat{\mathcal{A}_{E}}:=\left(\mathfrak{g} \otimes R_{E}\right) \oplus \Omega_{R_{E}}^{1} / d R_{E},
$$

é chamada de álgebra afim elíptica.

\footnotetext{
${ }^{5}$ Aqui $\operatorname{Im} \lambda$ indica a parte imaginária de $\lambda$
} 
A descrição de uma base para o centro de $\widehat{\mathcal{A}_{E}}$ também é conhecida. Para apresentar tais relações é necessário definir as seguintes famílias de polinômios $p_{k}(b), q_{k}(b)$ com $k \in \mathbb{Z}$ de forma que

$$
\overline{t^{k-2} u d t}=p_{k}(b) \overline{t^{k-1} u d t}+q_{k}(b) \overline{t^{-2} u d t} .
$$

Lema 3.7.25 ([Bre94], Lema 4.4) Os polinômios $p_{k}(b)$ e $q_{k}(b)$ são polinômios de Pollaczek com parâmetros $\lambda=-1 / 2, \alpha=0, \beta=-1$ e $\gamma=1 / 2$. As condições iniciais são

$$
p_{0}(b)=0, \quad p_{1}(b)=1, \quad q_{0}(b)=1, \quad \text { e } \quad q_{1}(b)=0 .
$$

A descrição de $\widehat{\mathcal{A}_{E}}$ é apresentada a seguir. Consideremos $\omega_{0}=\overline{t^{-1} d t}, \omega_{+}=\overline{t^{-1} u d t}$ e $\omega_{-}=\overline{t^{-2} u d t}$.

Teorema 3.7.26 ([Bre94], Teorema 4.6) A álgebra afim elíptica $\widehat{\mathcal{A}_{E}}$ tem uma $\mathbb{Z} / 2 \mathbb{Z}$-graduação em que

$$
{\widehat{\mathcal{A}_{E}}}^{0}=\mathfrak{g} \otimes \mathbb{C}\left[t, t^{-1}\right] \oplus \mathbb{C} \omega_{0}, \quad e \quad{\widehat{\mathcal{A}_{E}}}^{1}=\mathfrak{g} \otimes \mathbb{C}\left[t, t^{-1}\right] u \oplus \mathbb{C} \omega_{+} \oplus \mathbb{C} \omega_{-} .
$$

Um conjunto gerador de $\widehat{\mathcal{A}_{E}}$ consiste dos elementos $x \otimes t^{i}$ e $x \otimes t^{j-1} u$, em que $x \in \mathfrak{g}$, $i, j \in \mathbb{Z}$, com os elementos centrais $\omega_{0}, \omega_{+}$and $\omega_{-}$. A subálgebra par ${\widehat{\mathcal{A}_{E}}}^{0}$ é uma álgebra de Lie afim de Kac-Moody não torcida com as seguintes relações de comutatividade

$$
\begin{aligned}
{\left[x \otimes t^{i-1} u, y \otimes t^{j-1} u\right] } & =[x, y] \otimes\left(t^{i+j-1}-2 b t^{i+j}+t^{i+j+1}\right) \\
& +(x, y)\left\{\begin{array}{l}
-2 j b \omega_{0}, \text { se } i+j=0 \\
\frac{1}{2}(j-1) \omega_{0}, \text { se }|i+j|=1 \\
0, \text { se }|i+j| \geq 2 .
\end{array}\right.
\end{aligned}
$$

O subespaço impar ${\widehat{\mathcal{A}_{E}}}^{1}$ é um ${\widehat{\mathcal{A}_{E}}}^{0}$-módulo com relações dadas por

$$
\left[x \otimes t^{i-1} u, y \otimes t^{j} u\right]=[x, y] \otimes t^{i+j-1} u+(x, y) j\left(p_{|i+j|}(b) \omega_{+}+q_{|i+j|}(b) \omega_{-}\right) .
$$

\section{O caso DJKM}

Nesta subseção, apresentaremos a álgebra de Lie afim de Date-Jimbo-KashiwaraMiwa, ou simplesmente álgebra afim DJKM . Considere, nesta subseção, $\mathfrak{g}$ uma álgebra de Lie complexa de dimensão finita e simples.

Definição 3.7.27 O anel DJKM $R_{D J K M} e ́$ definido por $R_{D J K M}=\mathbb{C}\left[t, t^{-1}, u\right] /\left\langle u^{2}-\left(t^{2}-\right.\right.$ $\left.\left.a^{2}\right)\left(t^{2}-b^{2}\right)\right\rangle$, em que $a \neq \pm b$ são constantes complexas não nulas. Apesar de o anel DJKM satisfazer a curva superelíptica $u^{2}=\left(t^{2}-a^{2}\right)\left(t^{2}-b^{2}\right)$, as motivações para definição desta estrutura vem do trabalho de Date, Jimbo, Kashiwara e Miwa sobre a equação de LandauLifshitz em [Dat+83b]. Este anel é do tipo superelíptico.

Definição 3.7.28 O espaço vetorial $\mathcal{A}_{D J K M}=\mathfrak{g} \otimes R_{D J K M}$, munido das relações de comutatividade

$$
[x \otimes f, y \otimes g]=[x, y] \otimes f g
$$

em que $x, y \in \mathfrak{g}$ e $f, g \in R_{D J K M}$ é uma álgebra de Lie denominada álgebra laço DJKM

Nos concentraremos no estudo da extensão central universal da álgebra de Lie $\mathfrak{g} \otimes$ $R_{D J K M}$. 
Definição 3.7.29 A extensão central universal de $\mathcal{A}_{D J K M}$, a álgebra de Lie definida por

$$
\widehat{\mathcal{A}_{D J K M}}:=\left(\mathfrak{g} \otimes R_{D J K M}\right) \oplus \Omega_{R_{D J K M}}^{1} / d R_{D J K M},
$$

é chamada de álgebra afim DJKM.

De forma muito parecida que nos casos anteriores, é necessário definir uma família de polinômios $P_{k}:=P_{k}(c)$ dados por recursividade, para descrever as relações de comutatividade em $\widehat{\mathcal{A}_{D J K M}}$. Esta família é dada pela seguinte relação

$$
(6+2 k) P_{k}(c)=4 k c P_{k-2}(c)-2(k-3) P_{k-4}(c)
$$

em que $c=\left(a^{2}+b^{2}\right) / 2$ e $k \geq 0$.

Em posse do Teorema 3.7.20,temos que $\left\{\overline{t^{-1} d t}, \overline{t^{-1} u d t}, \overline{t^{-2} u d t}, \overline{t^{-3} u d t}, \overline{t^{-4} u d t}\right\}$ é uma base para $\Omega_{R_{D J K M}}^{1} / d R_{D J K M}$. Seja $\omega_{0}=\overline{t^{-1} d t}$ e $\omega_{-k}=\overline{t^{-k} u d t}$ para $k=1,2,3,4$. Temos o seguinte teorema que descreve as relações de comutatividade nesta álgebra.

Teorema 3.7.30 ([CF11], Teorema 4.0.2) Seja $(\cdot, \cdot)$ a forma de Killing em ge defina $\psi_{i j}(c) \in$ $\Omega_{R_{D J K M}}^{1}$ como

$$
\psi_{i j}(c)=\left\{\begin{array}{lll}
\omega_{i+j-2} & \text { para } i+j=1,0,-1,-2, \\
P_{-3, i+j-2}(c)\left(\omega_{-3}+c \omega_{-1}\right) & \text { para } i+j=2 n-1 \geq 3, n \in \mathbb{Z}, \\
P_{-3, i+j-2}(c)\left(c \omega_{-3}+\omega_{-1}\right) & \text { para } i+j=-2 n+1 \leq-3, n \in \mathbb{Z}, \\
\left.P_{-4,|i+j|-2}(c) \omega_{-4}+P_{|i+j|-2}(c) \omega_{-2}\right) & \text { para }|i+j|=2 n \geq 2, n \in \mathbb{Z} .
\end{array}\right.
$$

A álgebra de Lie $\widehat{\mathcal{A}_{D J K M}}$ tem uma $\mathbb{Z} / 2 \mathbb{Z}$-graduação

$$
\widehat{\mathcal{A}_{D J K M}}={\widehat{\mathcal{\mathcal { A } _ { D J K M }}}}^{0} \oplus{\widehat{\mathcal{\mathcal { A } _ { D J K M }}}}^{1}
$$

em que

$$
\begin{aligned}
& {\widehat{\mathcal{A}_{D J K M}}}^{0}=\left(\mathfrak{g} \otimes \mathbb{C}\left[t, t^{-1}\right]\right) \oplus \mathbb{C} \omega_{0}, \\
& {\widehat{\mathcal{A}_{D J K M}}}^{1}=\left(\mathfrak{g} \otimes \mathbb{C}\left[t, t^{-1}\right] u\right) \oplus \mathbb{C} \omega_{-4} \oplus \mathbb{C} \omega_{-3} \oplus \mathbb{C} \omega_{-2} \oplus \mathbb{C} \omega_{-1} .
\end{aligned}
$$

O colchete de Lie é dado por

$$
\begin{aligned}
{\left[x \otimes t^{i}, y \otimes t^{j}\right]=} & {[x, y] \otimes t^{i+j}+\delta_{i+j, 0} j(x, y) \omega_{0}, } \\
{\left[x \otimes t^{i-1} u, y \otimes t^{j-1} u\right]=} & {[x, y] \otimes\left(t^{i+j+2}-2 c t^{i+j}+t^{i+j-2}\right) } \\
& +\left(\delta_{i+j,-2}(j+1)+2 c j \delta_{i+j, 0}+(j-1) \delta_{i+j, 2}\right)(x, y) \omega_{0}, \\
{\left[x \otimes t^{i-1} u, y \otimes t^{j}\right]=} & {[x, y] u \otimes t^{i+j-1}+j(x, y) \psi_{i j}(c) . }
\end{aligned}
$$

Também é conhecida uma descrição para a álgebra $\widehat{\mathcal{A}_{D J K M}}$.

Teorema 3.7.31 ([CJ14], Teorema 3) A álgebra de Lie DJKM D é isomorfa à seguinte álgebra dada por geradores e relações. Considere os geradores 
$e_{n}, e_{n}^{1}, f_{n}, f_{n}^{1}, h_{n}, h_{n}^{1}, n \in \mathbb{Z}, w_{0}, w_{-1}, w_{-2}, w_{-3}, w_{-4}$ e relações dadas por

$$
\begin{aligned}
{\left[x_{m}, x_{n}\right]:=\left[x_{m}, x_{n}^{1}\right]=\left[x_{m}^{1}, x_{n}^{1}\right]=0, \text { para } x=e, f, } \\
{\left[h_{m}, h_{n}\right]:=-2 m \delta_{m,-n} \omega_{0}=(n-m) \delta_{m,-n} \omega_{0}, } \\
{\left[h_{m}^{1}, h_{n}^{1}\right]:=2\left((n+2) \delta_{m+n,-4}-2 c(n+1) \delta_{m+n,-2}+n \delta_{m+n, 0}\right) \omega_{0}, } \\
{\left[h_{m}, h_{n}^{1}\right]:=-2 m \psi_{m, n}(c), } \\
{\left[\omega_{i}, x_{m}\right]:=\left[\omega_{i}, \omega_{k}\right]=0 \text { para } x=e, f, h e i, j \in\{0,1\}, } \\
{\left[e_{m}, f_{n}\right]:=h_{m+n}-m \delta_{m,-n} \omega_{0}, } \\
{\left[e_{m}, f_{n}^{1}\right]:=h_{m+n}^{1}-m \psi_{m n}(c)=:\left[e_{m}^{1}, f_{n}\right], } \\
{\left[e_{m}^{1}, f_{n}^{1}\right]:=h_{m+n+4}-2 c h_{m+n+2}+h_{m+n} } \\
{\left[h_{m}, e_{n}\right]:=2 e_{m+n,} } \\
{\left[h_{m}, e_{n}^{1}\right]:=2 e_{m+n}^{1}=:\left[h_{n}^{1}, e_{m}\right], } \\
{\left[h_{m}^{1}, e_{n}^{1}\right]:=2 e_{m+n+4}-4 c e_{m+n+2}+2 e_{m+n}, } \\
{\left[h_{m}, f_{n}\right]:=-2 f_{m+n}, } \\
{\left[h_{m}, f_{n}^{1}\right]:=-2 f_{m+n}^{1}=:\left[h_{n}^{1}, f_{m}\right], } \\
{\left[h_{m}^{1}, f_{n}^{1}\right]:=-2 f_{m+n+4}+4 c f_{m+n+2}-2 f_{m+n}, }
\end{aligned}
$$

para todo $m, n \in \mathbb{Z}$.

\section{8 Álgebras de Lie afim superelípticas}

As curvas hiperelípticas são o caso mais simples de curvas superelípticas $u^{m}=k(t)$, em que $k(t) \in \mathbb{C}\left[t, t^{-1}, u\right]$ e $m \geq 2$.

Dado que as álgebras superelípticas são uma generalização das álgebras hiperelípticas, há uma pergunta natural que motiva este trabalho de pesquisa: a teoria desenvolvida para curvas hiperelípticas pode ser estendida para o caso das curvas superelípticas?

Apesar do tratamento algébrico que este trabalho empenha, a intuição geométrica e geométrica-algébrica permeia todo o percurso até aqui. Por este motivo, antes de tratar do contexto algébrico, é importante destacar que esta pergunta também emerge do contexto da geometria-algébrica. Em trabalho recente de Beshaj, Shaska, Zhupa em [BSZ15] e de Malmendier e Shaska em [MS19] é exibido que a teoria das curvas elípticas e hiperelípticas pode ser estendida naturalmente para todas as curvas superelípticas.

Considere as álgebras superelípticas a seguir:

$$
\begin{aligned}
R_{m}(k) & =\mathbb{C}\left[t, t^{-1}, u \mid u^{m}=k(t)\right] \mathrm{e} \\
S_{m}(k) & =\mathbb{C}\left[t, u \mid u^{m}=k(t)\right] .
\end{aligned}
$$

O seguinte resultado oferece as condições necessárias e suficientes em termos das multiplicidades de raízes de $k(t)$ para que $\mathcal{R}_{m}(k)=\operatorname{Der}\left(R_{m}(P)\right)$ e $\mathcal{S}_{m}(k)=\operatorname{Der}\left(S_{m}(P)\right)$ sejam álgebras de Lie simples de dimensão infinita.

Teorema 3.8.1 ([Cox+17], Teorema 4) Suponha que $m \geq 2$ e deg $(k) \geq 1$.

a) A álgebra de Lie $\mathcal{S}_{m}(k)$ é simples se, e somente se, $k(t)$ não tem raízes múltiplas. 
b) A álgebra de Lie $\mathcal{R}_{m}(k)$ é simples se, e somente se, $k(t)$ não tem raízes múltiplas não nulas e $k(t) \neq c t^{r}$ para todo $c \in \mathbb{C} \backslash\{0\}$ e $r \in \mathbb{Z}_{+}$em que $m d c(r, m) \neq 1$.

Assumindo que $\mathcal{R}_{m}(k)$ e $\mathcal{S}_{m}(k)$ são simples, é possível determinar as extensões centrais universais destas álgebras.

Teorema 3.8.2 ([Cox+17], Teorema 9) Suponha que $R=R_{m}(k)$ com $m \geq 2$ e $k(t)=$ $t^{L}\left(t-\alpha_{1}\right) \cdots\left(t-\alpha_{D}\right)$ para algum $L \in \mathbb{Z}, D \in \mathbb{N}$ e complexos $\alpha_{1}, \ldots, \alpha_{D} \in \mathbb{C} \backslash\{0\}$ dois a dois distintos. Então a extensão central universal de $\mathcal{R}_{m}(k)$ é $\mathcal{R}_{m}(k) \oplus R / \partial(R)$ com colchetes dados por

$$
[f \partial, g \partial]=f \partial(g) \partial-g \partial(f)+\overline{\partial(f) \partial(\partial(g))}, \forall f, g \in R
$$

em que $\partial=\sqrt[m]{k(t)^{m-1}} \frac{d}{d t}$. Além disso,

$$
\operatorname{dim}(R / \partial(R))=1+D(m-1) .
$$

Considere, a partir daqui, $R:=\mathbb{C}\left[t, t^{-1}, u\right]$ em que $u^{m} \in \mathbb{C}[t]$, com $m \geq 2$. Sobre o polinômio $u^{m}=p(t) \in \mathbb{C}[t]$, vamos assumir que

$$
p(t)=\sum_{i=0}^{D} a_{i} t^{i}
$$

com $D \in \mathbb{N},\left\{a_{1}, \ldots, a_{D}\right\} \subset \mathbb{C}$ sendo $a_{0}$ e $a_{1}$ não ambos nulos e com $a_{D}=1$. Também vamos impor que $p(t)$ tem raízes distintas de multiplicidade 1 , isto é,

$$
p(t)=\left(t-\alpha_{1}\right) \cdots\left(t-\alpha_{D}\right)
$$

em que $\left\{\alpha_{1}, \ldots, \alpha_{D}\right\} \subset \mathbb{C}$, sendo estes $\alpha_{i}$ s dois a dois distintos. Observe que se $a_{0} \neq 0$ o polinômio $p(t)$ tem $D$ raízes não nulas de multiplicidade igual a 1 . Caso $a_{0}=0$, teremos que $p(t)$ possui $(D-1)$ raízes não nulas. Esta observação será utilizada em breve quando exibirmos a dimensão de $\Omega_{R}^{1} / d R$.

É fácil ver que $R$ tem uma base consistindo de $t^{i}, t^{i} u, t^{i} u^{2}, \ldots, t^{i} u^{m-1}$ com $i \in \mathbb{Z}$. Note também que $u^{m}=p(t)$ define uma curva superelíptica. Se definirmos $R^{i}$ para $\mathbb{C}\left[t, t^{-1}\right] u^{i}$, então podemos observar a seguinte $\mathbb{Z} / m \mathbb{Z}$-graduação para o anel $R$ :

$$
R=R^{0} \oplus R^{1} \oplus \cdots \oplus R^{m-1} .
$$

Além disso, considere aqui $\mathfrak{g}$ uma álgebra de Lie complexa, simples e de dimensão finita. As álgebras $\mathcal{G}=\mathfrak{g} \otimes R$ são exemplos de álgebras superelípticas afim. A $\mathbb{Z} / m \mathbb{Z}$-graduação de $R$ dada em (3.7) induz em $\mathcal{G}$ uma estrutura de álgebra de Lie $\mathbb{Z} / m \mathbb{Z}$-graduada definindo

$$
\mathcal{G}^{i}=\mathfrak{g} \otimes R^{i} \text { em que } i \in\{0,1, \ldots, m-1\} .
$$

Definindo $\hat{\mathcal{G}}$ como a extensão central universal de $\mathcal{G}$, então como espaços vetoriais temos que $\hat{\mathcal{G}}=\mathcal{G} \oplus C$, em que $C$ é o núcleo do homomorfismo sobrejetor de $\hat{\mathcal{G}}$ em $\mathcal{G}$. Isto significa que $C$ é o centro de $\hat{\mathcal{G}}$. Utilizando o Teorema 3.7.6, sabemos que o centro $C$ de $\hat{\mathcal{G}}$ é linearmente isomorfo a $\Omega_{R} / d R$, o espaço de diferenciais de Kähler de $R$ módulo diferenciais exatos. Conforme vimos na Seção 3.7.4, no contexto das álgebras de Lie elípticas afim, são conhecidos os seguintes aspectos sobre estas estruturas:

1. uma descrição de $C$, em particular sua dimensão, 
2. uma base para $C$, e

3. o cociclo universal $\hat{\mathcal{G}} \times \hat{\mathcal{G}} \rightarrow C$ explicitamente.

Estudaremos estas três situações para o caso mais geral de álgebras de Lie superelípticas afim, retomando a pergunta destacada no início desta subseção, queremos investigar o que podemos estender para o caso de curvas superelípticas. Verificaremos a seguir que é possível estender alguns resultados obtidos do o caso hiperelíptico para o caso superelíptico.

Como seguiremos utilizando as definições e notações estabelecidas quando construímos o espaço dos diferenciais de Kähler no inicio deste capítulo, vamos retomálas:

1. K é o submódulo de $R \otimes R$ gerado por elementos $1 \otimes f g-f \otimes g-g \otimes f$;

2. $F=R \otimes R$ é um $R$-módulo à esquerda com ação $f(g \otimes h)=f g \otimes h$ em que $f, g, h \in R$;

3. $\Omega_{R}^{1}=F / K$ é o módulo de diferenciais de $R$;

4. Denotaremos o elemento $f \otimes g+K$ de $\Omega_{R}^{1}$ por $f d g$;

5. Definiremos uma aplicação $d: R \rightarrow \Omega_{R}^{1}$ por $d(f)=1 \otimes f+K$;

6. Denotaremos a coclasse $f d g$ módulo $d R$ por $\overline{f d g}$.

Como é possível realizar $R$ como $\mathbb{C}\left[t, t^{-1}, \sqrt[m]{p(t)}\right]$ identificando $u$ com $\sqrt[m]{p(t)}$, então $\mathcal{R}_{m}(p)=R_{m}(p) \partial$ e $\operatorname{dim} \Omega_{R}^{1} / d R=\operatorname{dim} R / \partial R$. Como consequência direta da equação (3.5), e observando o polinômio (3.6), temos o primeiro e mais simples resultado:

$$
\operatorname{dim} \Omega_{R}^{1} / d R=\left\{\begin{array}{l}
1+D(m-1) \text { se } a_{0} \neq 0 \\
1+(D-1)(m-1) \text { se } a_{0}=0
\end{array}\right.
$$

Para demonstrar, basta observar que quando $a_{0}=0$, o polinômio $p(t)$ "ganha" uma raiz zero e "perde" uma raiz não nula.

Lema 3.8.3 $\Omega_{R}^{1}$ é gerado pelos diferenciais $t^{i} u^{k} d t$ e $t^{i} u^{l} d u$ em que $i \in \mathbb{Z}, k \in\{0,1, \ldots, m-$ $1\}$, e $l \in\{0,1, \ldots, m-2\}$.

Demonstração: Temos que mostrar que qualquer elemento de $R \otimes R$ é congruente módulo $K$ a um elemento no espaço gerado por $t^{i} u^{k} \otimes t$ e $t^{i} u^{l} \otimes u \operatorname{com} i \in \mathbb{Z}, k \in$ $\{0,1, \ldots, m-1\}$, e $l \in\{0,1, \ldots, m-2\}$. Note que, por indução

$$
d\left(t^{j} u^{l}\right)=j t^{j-1} u^{l} d t+l t^{j} u^{l-1} d u .
$$

Como $K$ é um submódulo de $R \otimes R$, podemos multiplicar cada um dos membros de (3.8) por $t^{i} u^{k}$ obtendo

$$
t^{i} u^{k} d\left(t^{j} u^{l}\right)=j t^{i+j-1} u^{k+l} d t+l t^{i+j} u^{k+l-1} d u .
$$

Como $u^{m-1} d u=\frac{1}{m} d\left(u^{m}\right) \Rightarrow t^{i} u^{m-1} d u=\frac{1}{m} D t^{i+D-1} d t+\frac{1}{m}(D-1) a_{D-1} t^{i+D-2} d t+$ $\cdots+\frac{1}{m} a_{1} t^{i} d t$. Isso nos mostra que qualquer elemento na base de $R \otimes R$ é igual a um elemento no espaço gerado por $t^{i} u^{k} d t$ e $t^{i} u^{l} d u$ em que $k \in\{0,1, \ldots, m-1\}$ e $l \in\{0,1, \ldots, m-2\}$.

Lema 3.8.4 $\Omega_{R}^{1}$ é gerado pelos diferenciais $t^{i} d t, t^{i} u d t, \ldots, t^{i} u^{m-1} d t$, em que $i \in \mathbb{Z}$, juntos $\operatorname{com} t^{D-1} u^{l} d u, \ldots, t u^{l} d u, u^{l} d u$ (aqui omitimos $u^{l} d u$ se $a_{0}=0$ ), com $l \in\{0,1, \ldots, m-2\}$. 
Demonstração: Temos que $\frac{1}{m} u d\left(u^{m}\right)=u^{m} d u$. Como $u^{m}=\sum_{k=0}^{D} a_{k} t^{k}$ então,

$$
\sum_{k=1}^{D} \frac{1}{m} k a_{k} t^{k-1} u d t-\sum_{k=0}^{D} a_{k} t^{k} d u=0
$$

Multiplicando a equação (3.9) por $t^{i}$ obtemos

$$
\sum_{k=1}^{D} \frac{1}{m} k a_{k} t^{i+k-1} u d t-\sum_{k=0}^{D} a_{k} t^{i+k} d u=0 .
$$

Caso $a_{0} \neq 0$. Para $i \geq 0$, a fórmula (3.10) mostra que (como $a_{D}=1$ ) $t^{i+D} d u$ é combinação linear de $t^{i+D-1} d u, \ldots, t^{i} d u$ e elementos da forma $t^{j} u d t$. Se $i<0$, temos que $\left(\right.$ como $\left.a_{0} \neq 0\right) t^{i} d u$ é combinação linear de elementos da forma $t^{i+1} d u, \cdots, t^{i+D} d u \mathrm{e}$ elementos da forma $t^{j} u d t$. Portanto, por indução, temos que os elementos da forma $t^{i} d u$ são iguais a combinações lineares de $d u, \ldots, t^{D-1} d u$ e elementos da forma $t^{j} u d t$.

Caso $a_{0}=0$ então para $i \geq 0, t^{i+D} d u$ é igual a combinação linear de elementos $t^{i+D-1} d u, \ldots, t^{i+1} d u$ e elementos da forma $t^{j} u d t$, e para $i<0$, como $a_{1} \neq 0, t^{i+1} d u$ é igual a combinação linear de $t^{i+2} d u, \ldots, t^{i+D} d u$ e elementos da forma $t^{j} u d t$. O restante do argumento é similar.

Podemos multiplicar a fórmula (3.9) por $t^{i} u^{l}$ para obter que

$$
\sum_{k=1}^{D} \frac{1}{m} k a_{k} t^{i+k-1} u^{1+l} d t-\sum_{k=0}^{D} a_{k} t^{i+k} u^{l} d u=0 .
$$

Similarmente, utilizando indução podemos mostrar que elementos da forma $t^{i} u^{l} d u$ são combinações lineares de $u^{l} d u, \ldots, t^{D-1} u^{l} d u$ (em que omitimos $u^{l} d u$ se $a_{0}=0$ ), e elementos da forma $t^{j} u^{l} d t$. Utilizando o Lema 3.8.3 completamos a demonstração.

Munidos do Lema 3.8.4 e a equação (3.5), temos condições de descrever uma base finita para $\Omega_{R}^{1} / d R$.

Teorema 3.8.5 Uma base finita para $\Omega_{R}^{1} / d R$ é dada por $\overline{t^{-1} d t} \operatorname{com} \overline{t^{-1} \mathcal{u}^{l} d t}, \ldots, \overline{t^{-D} \mathcal{u}^{l} d t}$ (em que omitimos $\overline{t^{-D} u^{l} d t}$ se $a_{0}=0$ ), com $l \in\{1,2, \ldots, m-1\}$.

Demonstração: Da $\mathbb{Z} / m \mathbb{Z}$-graduação de $\Omega_{R}^{1}$ e $d R$ resulta que

$$
\Omega_{R}^{1} / d R=\bigoplus_{i=0}^{m-1}\left(\Omega_{R}^{1}\right)^{i} / d\left(R^{i}\right)
$$

em que se $k \geq 1,\left(\Omega_{R}^{1}\right)^{k} / d\left(R^{k}\right)=\left\langle\overline{t^{j} u^{k} d t}, \overline{t^{D-1} u^{k-1} d u}, \ldots, \overline{t u^{k-1} d u}, \overline{u^{k-1} d u} \mid j \in \mathbb{Z}\right\rangle$ (omitimos $\overline{u^{k-1} d u}$ se $a_{0}=0$ ) e $\left(\Omega_{R}^{1}\right)^{0} / d\left(R^{0}\right):=\left\langle\overline{t^{j} d t} \mid j \in \mathbb{Z}\right\rangle$.

Analisaremos primeiro o espaço $\left(\Omega_{R}^{1}\right)^{0} / d\left(R^{0}\right)$. Temos que $d\left(t^{i}\right)=i t^{i-1} d t$ para todo $i \in \mathbb{Z}$. A partir disso, obtemos que $t^{i-1} d t \equiv 0(\bmod d R)$ para $i \neq 0$. Portanto $\left(\Omega_{R}^{1}\right)^{0} / d\left(R^{0}\right)$ é gerado por $\overline{t^{-1} d t}$.

Agora, vamos considerar o espaço $\left(\Omega_{R}^{1}\right)^{1} / d\left(R^{1}\right)$. Este espaço é gerado por $\overline{t^{i} u d t}$ e $\overline{t^{D-1} d u}, \ldots, \overline{t d u}\left(\mathrm{e} \overline{d u}\right.$ se $\left.a_{0} \neq 0\right)$. Temos que $d\left(t^{i} u\right)=i t^{i-1} u d t+t^{i} d u$, portanto,

$$
t^{i} d u \equiv-i t^{i-1} u d t(\bmod d R) .
$$


Então, nós precisamos considerar apenas os elementos do tipo $t^{i} u d t$. Nós vamos mostrar que módulo $d R$ cada um destes elementos é congruente à uma combinação linear finita de elementos listados no enunciado do Teorema 3.8.5.

Primeiro suponha que $a_{0} \neq 0$. Temos que $t^{i-1} u d t \equiv-(1 / i) t^{i} d u(\bmod d R)$ para $i \neq 0$. Pela fórmula (3.10), sabemos que $t^{i} d u$ é uma combinação linear de $t^{i+1} d u, \ldots, t^{i+D} d u$ e $t^{i} u d t, \ldots, t^{i+D-1} d t$. Utilizando (3.13), vemos que $t^{i} d u$, portanto $t^{i-1} u d t$, é congruente módulo $d R$ a um elemento gerado por $t^{i} u d t, \ldots, t^{i+D-1} u d t$. Utilizando a indução, vemos que para $i \leq-D-1$, o elemento $t^{i-1} u d t$ é congruente módulo $d R$ a uma combinação linear de $t^{-D} u d t, \ldots, t^{-1} u d t$.

Temos também que $t^{i+D-1} u d t \equiv-(1 /(i+d)) t^{i+D} d u(\bmod d R)$ para $i \neq-d$. Pela fórmula (3.10), novamente, obtemos que $t^{i+D} d u$ é uma combinação linear de $t^{i+D-1} d u, \ldots, t^{i} d u$ e $t^{i+D-1} u d t, \ldots, t^{i} u d t$. O coeficiente de $t^{i+D-1} u d t$ nessa combinação linear é $(D / m)$; portanto, podemos resolver para $t^{i+D-1} u d t$, mostrando que $t^{i+D-1} u d t$ é congruente módulo $d R$ a uma combinação linear dos mesmos elementos (excluindo $t^{i+D-1} u d t$ ). Pela fórmula (3.13) vemos que $t^{i+D-1} u d t$ é congruente módulo $d R$ a uma combinação linear de $t^{i+D-2} u d t, \ldots, t^{i-1} u d t$. Definindo $j=i+D-1$ e usando indução, vemos que para $j \geq 0$ (isto é $i \geq-D+1$ ), o elemento $t^{j} u d t$ é congruente módulo $d R$ à uma combinação linear de $t^{-1} u d t, \ldots, t^{-D} u d t$.

A demonstração para o caso em que $a_{0}=0\left(\right.$ e $\left.a_{1} \neq 0\right)$ é similar.

Consideraremos, agora, os espaços $\left(\Omega_{R}^{1}\right)^{l} / d\left(R^{l}\right)$ em que $l \in\{2,3, \ldots, m-1\}$. Cada $\left(\Omega_{R}^{1}\right)^{l} / d\left(R^{l}\right)$ é gerado por $t^{i} u^{l} d t$ e $t^{D-1} u^{l-1} d u, \ldots, t u^{l-1} d u\left(\right.$ e $u^{l-1} d u$ se $\left.a_{0} \neq 0\right)$. Temos que $d\left(t^{i+1} u^{l}\right)=(i+1) t^{i} u^{l} d t+l t^{i+1} u^{l-1} d u$, enão

$$
t^{i} u^{l} d t \equiv \frac{-l}{i+1} t^{i+1} u^{l-1} d u(\bmod d R)
$$

Então, nós precisamos considerar apenas os elementos do tipo $t^{i} u^{l} d u$.

Suponha que $a_{0} \neq 0$. Teremos que $t^{i} u^{l-1} d u \equiv(-i / l) t^{i-1} u^{l} d t(\bmod d R)$ para $i \neq 0$. Pela fórmula (3.11), sabemos que $t^{i} u^{l-1} d u$ é uma combinação linear de $t^{i+1} u^{l-1} d u, \ldots, t^{i+D} u^{l-1} d u$ e $t^{i} u^{l} d t, \ldots, t^{i+D-1} u^{l} d t$. Utilizando (3.14), vemos que $t^{i+1} u^{l-1} d u$, e portanto $t^{i} u^{l} d t$ também, é congruente módulo $d R$ a um elemento gerado por uma combinação linear de $t^{i} u^{l} d t, \ldots, t^{i+D-1} u^{l} d t$. Utilizando indução, obtemos que para $i \leq-D-1$, o elemento $t^{i-1} u^{l} d t$ é congruente módulo $d R$ a uma combinação linear de $t^{-D} u^{l} d t, \ldots, t^{-1} u^{l} d t$.

Temos que $t^{i+D-1} u^{l} d t \equiv-(l /(i+D)) t^{i+D} u^{l-1} d u(\bmod d R)$ para $i \neq-D$. Pela fórmula (3.11), novamente, mostramos que $t^{i+D} u^{l-1} d u$ é uma combinação linear de $t^{i+D-1} u^{l-1} d u, \ldots, t^{i} u^{l-1} d u$ e $t^{i+D-1} u^{l} d t, \ldots, t^{i} u^{l} d t$. O coeficiente de $t^{i+D-1} u^{l} d t$ nessa combinação linear é $D / m$; portanto, podemos resolver para $t^{i+D-1} u^{l-1} d t$, mostrando que $t^{i+D-1} u^{l-1} d t$ é congruente módulo $d R$ a uma combinação linear dos mesmos elementos (excluindo $t^{i+D-1} u^{l-1} d t$ ). Pela fórmula (3.14) temos que $t^{i+D-1} u^{l} d t$ é congruente módulo $d R$ a uma combinação linear de $t^{i+D-2} u^{l} d t, \ldots, t^{i-1} u^{l} d t$. Definindo $j=i+D-1$ e usando indução, teremos que para $j \geq 0$ (isto é $i \geq-D+1$ ), o elemento $t^{j} u^{l} d t$ é congruente módulo $d R$ a uma combinação linear de $t^{-1} u^{l} d t, \ldots$, $t^{-D} u^{l} d t$.

A demonstração para o caso em que $a_{0}=0$ (and $\left.a_{1} \neq 0\right)$ é similar.

Portanto, $\left(\Omega_{R}^{1}\right)^{l} / d\left(R^{l}\right)$ é gerado por $\overline{t^{-1} u^{l} d t}, \ldots, \overline{t^{-D} u^{l} d t}$ (em que omitimos $\overline{t^{-D} u^{l} d t}$ se $a_{0}=0$ ). A equação (3.5) completa a demonstração.

Para tornar as relações de comutatividade em $\hat{\mathcal{G}}$ explícitas, nós precisamos calcular $\overline{f d g}$ para todos elementos da base $f, g \in R$. Note que $\overline{f d g}$ é sempre uma combinação linear de elementos da base de $\Omega_{R}^{1} / d R$. Pelo Teorema 3.8.5 nós sabemos 
que os elementos $\overline{t^{-1} d t}$, e $\overline{t^{-1} u^{l} d t}, \ldots, \overline{t^{-D} u^{l} d t}$ (em que omitimos $\overline{t^{-D} u^{l} d t}$ se $a_{0}=0$ ), $\operatorname{com} l \in\{1,2, \ldots, m-1\}$ nos dão uma base para $\Omega_{R}^{1} / d R$.

Primeiro, apresentaremos a descrição dos cociclos que contribuem para a parte ímpar da álgebra de Lie afim superelíptica.

Sejam

$$
\omega_{0}=\overline{t^{-1} d t} \text { e } \omega_{i, j}=\overline{t^{i} u^{j} d t} \text { se } j \neq 0
$$

Proposição 3.8.6 ([Bre94], Proposição 4.2) Seja $b=-6 m /\left(12 m^{2}-60 \sum_{\xi \in \Lambda \backslash\{0\}} \xi^{-4}\right)^{2}$, então para $i, j \in \mathbb{Z}$, temos que

$$
\overline{t^{i} d\left(t^{j}\right)}=j \delta_{i+j, 0} \omega_{0}
$$

e

$$
\overline{t^{i-1} u d\left(t^{j-1} u\right)}=\left\{\begin{array}{l}
\left(j+\frac{1}{2}\right) \omega_{0}, \text { se } i+j=-1 \\
-2 j b \omega_{0}, \text { se } i+j=0 \\
\left(j-\frac{1}{2}\right) \omega_{0}, \text { se } i+j=1 \\
0 \text { se }|i+j| \geq 2
\end{array}\right.
$$

Proposição 3.8.7 Para $i, j \in \mathbb{Z} e l_{1}, l_{2} \in\{1,2, \ldots, m-1\}$, temos que

$$
\overline{t^{i} u^{l_{1}} d\left(t^{j} u^{l_{2}}\right)}=\left(\frac{j l_{1}-i l_{2}}{l_{1}+l_{2}}\right) \omega_{i+j-1, l_{1}+l_{2}} .
$$

Demonstração: O resultado segue da seguinte relação

$$
\begin{aligned}
\overline{t^{i} u^{l_{1}} d\left(t^{j} u^{l_{2}}\right)} & =j \overline{t^{i+j-1} u^{l_{1}+l_{2}} d t}+l_{2} \overline{t^{i+j} u^{l_{1}+l_{2}-1} d u} \\
& \equiv j \overline{t^{i+j-1} u^{l_{1}+l_{2}} d t}+l_{2}\left(\frac{-i-j}{l_{1}+l_{2}}\right) \overline{t^{i+j-1} u^{l_{1}+l_{2}} d t} \\
& =\left(j-\frac{l_{2}(i+j)}{l_{1}+l_{2}}\right) \overline{t^{i+j-1} u^{l_{1}+l_{2}} d t} \\
& =\left(\frac{j l_{1}-i l_{2}}{l_{1}+l_{2}}\right) \overline{t^{i+j-1} u^{l_{1}+l_{2}} d t} .
\end{aligned}
$$

Lema 3.8.8 Se $R=\mathbb{C}\left[t, t^{-1}, u\right] \operatorname{com} u^{m}=p(t) \in \mathbb{C}[t]$ com grau $D$, então vale que

$$
t^{n} u^{l} d t \equiv \sum_{k=0}^{D-1}\left(-a_{k}\left(\frac{k l+(-D+1+n+k) m}{D l+(1+n) m}\right)\right) t^{-D+n+k} u^{l} d t(\bmod d R)
$$

ou

$$
t^{n} u^{l} d t \equiv\left\{\begin{array}{l}
\sum_{k=1}^{D}\left(-a_{k}\left(\frac{k l+(1+n+k) m}{a_{0}(1+n) m}\right)\right) \\
\sum_{k=2}^{D}\left(-a_{k}\left(\frac{k l+(1+n+k) m}{a_{1}(l+(i+1) m)}\right)\right) t^{n+k} u^{l} d t(\bmod d R) \text { se } a_{0} \neq 0 \text { e } n \leq-D-1 \\
t^{n+k} u^{l} d t(\bmod d R) \text { se } a_{0}=0 \text { e } n \leq-D-1
\end{array}\right.
$$

para $n \in \mathbb{Z} \backslash\{-1,-2, \cdots,-D\}$ (ou $n \in \mathbb{Z} \backslash\{-1,-2, \cdots,-D+1\}$, se $a_{0}=0$ ). 
Demonstração: Expandindo $d\left(t^{i+k} u^{l}\right)$, temos que

$$
-\left(\frac{i+k}{l}\right) t^{i+k-1} u^{l} d t \equiv t^{i+k} u^{l-1} d u(\bmod d R)
$$

a equação (3.11) implica que

$$
\sum_{k=0}^{D} \frac{1}{m} k a_{k} t^{i+k-1} u^{l} d t+\sum_{k=0}^{D} a_{k} t^{i+k-1} u^{l} d t \equiv 0(\bmod d R)
$$

ou

$$
\sum_{k=0}^{D} a_{k}\left(\frac{k l+(i+k) m}{l m}\right) t^{i+k-1} u^{l} d t \equiv 0(\bmod d R) .
$$

O que nos dá (3.18).

Definimos a sequência de polinômios em $D+3$ parâmetros $P_{m, n, l}\left(a_{0}, a_{1}, \ldots, a_{D-1}\right):=P_{n}$ por $n \geq-D, m, l \in \mathbb{Z}_{+}$e $a_{0}, a_{1}, \ldots, a_{D-1} \in \mathbb{C}$ da seguinte forma:

$$
(D l+(1+n) m) P_{n}=\sum_{k=0}^{D-1}\left(-a_{k}(k l+(-D+1+n+k) m)\right) P_{-D+n+k}
$$

com condições iniciais

$$
P_{-D}=t^{-D} u^{l} d t, P_{-D+1}=t^{-D+1} u^{l} d t, \ldots, P_{-1}=t^{-1} u^{l} d t
$$

Podemos verificar que, para $n \geq 0$

$$
\begin{aligned}
t^{0} u^{l} d t & =P_{0} ; \\
t^{1} u^{l} d t & =P_{1} \\
t^{2} u^{l} d t & =P_{2} ; \\
t^{3} u^{l} d t & =P_{3} ; \\
\vdots & \\
t^{n} u^{l} d t & =P_{n} .
\end{aligned}
$$

Caso $a_{0} \neq 0$ definimos uma sequência de polinômios em $D+3$ parâmetros $Q_{m, n, l}\left(a_{0}, a_{1}, \ldots, a_{D-1}\right):=Q_{n}$ para $n \leq-D-1, m, l \in \mathbb{Z}_{+}$e $a_{0}, a_{1}, \ldots, a_{D-1} \in \mathbb{C}$ por

$$
\left(a_{0}(1+n) m\right) Q_{n}=\sum_{k=1}^{D}\left(-a_{k}(k l+(1+n+k) m)\right) Q_{n+k}
$$

com as seguintes condições iniciais

$$
Q_{-D}=t^{-D} u^{l} d t, Q_{-D+1}=t^{-D+1} u^{l} d t, \ldots, Q_{-1}=t^{-1} u^{l} d t .
$$

Podemos também verificar que, para $n \leq-D-1$ e $a_{0} \neq 0$,

$$
t^{n} u^{l} d t=Q_{n} .
$$

Caso $a_{0}=0$ definimos uma sequência de polinômios em $D+3$ parâmetros $R_{m, n, l}\left(a_{0}, a_{1}, \ldots, a_{D-1}\right):=R_{n}$ para $n \leq-D-1, m, l \in \mathbb{Z}_{+}$e $a_{0}, a_{1}, \ldots, a_{D-1} \in \mathbb{C}$ por

$$
a_{1}(l+(1+n+1) m) R_{n}=\sum_{k=2}^{D}\left(-a_{k}(k l+(1+n+k) m)\right) R_{n+k}
$$


com as seguintes condições iniciais

$$
R_{-D+1}=t^{-D+1} u^{l} d t, R_{-D+2}=t^{-D+2} u^{l} d t, \ldots, R_{-1}=t^{-1} u^{l} d t .
$$

Podemos verificar que, para $n \leq-D-1$ e $a_{0}=0$,

$$
t^{n} u^{l} d t=R_{n}
$$

O Teorema 3.8.5 nos dá que $\overline{t^{-1} d t}, \operatorname{com} \overline{t^{-1} u^{l} d t}, \ldots, \overline{t^{-D} u^{l} d t}$ (em que omitimos $\overline{t^{-D} u^{l} d t}$ se $\left.a_{0}=0\right)$, com $l \in\{1,2, \ldots, m-1\}$ é uma base para $\Omega_{R}^{1} / d R$. É fácil verificar que as condições iniciais em (3.20), (3.22) e (3.20) nos dá que cada família $P_{n}, Q_{n}$ e $R_{n}$ é dada por uma combinação linear explícita desses elementos da base.

Proposição 3.8.9 Seja

$$
\psi_{i, j}=\left\{\begin{array}{l}
P_{i+j-1} \text { se } i+j \geq-D+1 \\
Q_{i+j-1} \text { se } i+j \leq-D \text { e } a_{0} \neq 0 \\
R_{i+j-1} \text { se } i+j \leq-D \text { e } a_{0}=0
\end{array}\right.
$$

então,

$$
t^{i} u^{l} d\left(t^{j}\right)=j \psi_{i, j}
$$

Demonstração: A demonstração segue do fato de que $t^{i} u^{l} d\left(t^{j}\right)=j t^{i+j-1} u^{l} d t$ e da definição de $P_{n}, Q_{n}$ e $R_{n}$. $\hat{\mathcal{G}}$.

Agora é possível dar uma descrição explícita das relações de comutatividade em

Teorema 3.8.10 A álgebra de Lie superelíptica afim $\hat{\mathcal{G}}$ tem uma $\mathbb{Z} / m \mathbb{Z}$-graduação tal que

$$
\hat{\mathcal{G}}^{0}=\mathfrak{g} \otimes \mathbb{C}\left[t, t^{-1}\right] \oplus \mathbb{C} \omega_{0}, \quad \hat{\mathcal{G}}^{l}=\mathfrak{g} \otimes \mathbb{C}\left[t, t^{-1}\right] u^{l} \bigoplus_{n=1}^{D} \mathbb{C} \omega_{-n, l} .
$$

A subalgebra $\hat{\mathcal{G}}^{0}$ é uma álgebra de Lie Kac-Moody afim não torcida com relações de comutatividade

$$
\left[x \otimes t^{i}, y \otimes t^{j}\right]=[x, y] \otimes t^{i+j} \oplus \delta_{i+j, 0}(x, y) j \omega_{0} .
$$

As relações de comutatividade em $\hat{\mathcal{G}}$ são

$$
\left[x \otimes t^{i} u^{l_{1}}, y \otimes t^{j} u^{l_{2}}\right]=[x, y] \otimes\left(t^{i+j} u^{l_{1}+l_{2}}\right)+(x, y)\left(\frac{j l_{1}-i l_{2}}{l_{1}+l_{2}}\right) \omega_{i+j-1, l_{1}+l_{2}}
$$

se $l_{1}+l_{2} \leq m-1$. Quando $l_{1}+l_{2}>m-1$,

$$
\left[x \otimes t^{i} u^{l_{1}}, y \otimes t^{j} u^{l_{2}}\right]=[x, y] \otimes\left(\sum_{k=0}^{D} a_{k} t^{i+j+k} u^{l_{1}+l_{2}-m}\right)+(x, y)\left(\frac{j l_{1}-i l_{2}}{l_{1}+l_{2}}\right) \omega_{i+j-1, l_{1}+l_{2}} .
$$

O subespaço $\hat{\mathcal{G}}^{l}$ é um $\hat{\mathcal{G}}^{0}$-módulo com relações dadas por

$$
\left[x \otimes t^{i} u^{n}, y \otimes t^{j}\right]=[x, y] \otimes\left(t^{i+j} u^{n+1}\right)+(x, y) j \psi_{i, j} .
$$




\section{Capítulo 4}

\section{Polinômios Ortogonais}

A origem do estudo de polinômios ortogonais pode ser atribuída ao trabalho do matemático francês Adrien-Marie Legendre (1752 - 1833) sobre o movimento planetário. Os polinômios de Legendre são exemplos de polinômios ortogonais com uma vasta gama de aplicações. O interesse pelo estudo de polinômios ortogonais foi crescente desde então e o trabalho do matemático húngaro-americano Gábor Szegő (1895 - 1985) veio a consagrar este tópico como de grande interesse pela comunidade científica.

Apesar deste trabalho ser centrado no estudo de estruturas algébricas, é importante salientar que os polinômios ortogonais possuem uma relação forte com vários campos importantes de análise. Além disso, algumas famílias de polinômios ortogonais se mostraram significantes em problemas de mecânica quântica e matemática estatística.

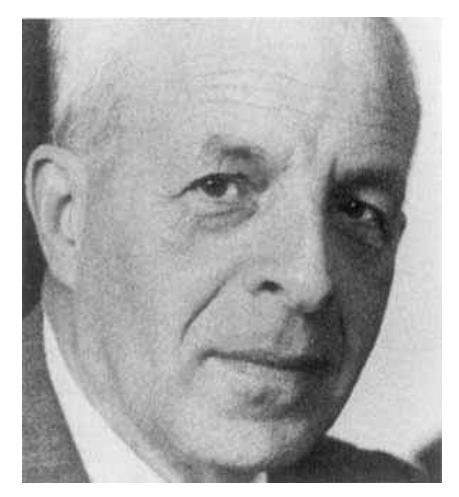

FIGURA 4.1: G. Szegő

Polinômios ortogonais estão relacionados à várias áreas importantes da análise, trigonometria, hipergeometria, funções de Bessel, funções elípticas e também aparecem na teoria de equações diferenciais e de equações integrais e durante o estudo das relações de comutatividade de alguns exemplos de álgebras de KricheverNovikov, emergem relações de recursividade que podem ser relacionadas à polinômios ortogonais.

A extensão central universal das álgebras DJKM possui relações de comutatividade descritas por expressões que dependem da definição de quatro famílias de polinômios ([CF11]). Duas destas famílias são dadas em termos de integrais elípticas e as outras duas são variações de polinômios ultraesféricos ([CFT13]) sendo que as famílias dadas por integrais elípticas satisfazem equações diferenciais lineares de ordem 4 e são formadas por polinômios ortogonais não-clássicos. Neste capítulo apresentaremos de forma concisa estes resultados já conhecidos.

Este capítulo apresenta como resultado principal a existência de uma nova família de polinômios ortogonais não-clássicos, originária das relações de recursividade obtidas no estudo das álgebras de Krichever-Novikov superelípticas (ver Seção 3.8), que satisfaz certa equação diferencial de ordem 4 . 


\subsection{Polinômios ortogonais}

Suponha que $w>0$ é uma função real definida num subconjunto suficientemente grande de $(a, b)$, então

$$
\int_{a}^{b} w(x) d x>0
$$

Se há uma sequência de polinômios $\left\{P_{n}(x)\right\}_{n=0}^{\infty}$ em que $P_{n}(x)$ tem grau $n$, tal que

$$
\int_{a}^{b} P_{n}(x) P_{m}(x) w(x) d x=0 \text { para } n \neq m,
$$

então $\left\{P_{n}(x)\right\}$ é chamada de família (ou sequência) de polinômios ortogonais com respeito à função peso $w$ em $(a, b)$.

Exemplo 4.1.1 Os polinômios de Jacobi, $P_{n}^{(\alpha, \beta)}(x)$, são polinômios ortogonais com respeito à função de peso $(1-x)^{\alpha}(1+x)^{\beta} \operatorname{com}(\alpha>-1, \beta>-1) \mathrm{em}(-1,1)$. Consideraremos as famílias definidas pela relação de recursividade

$$
\begin{aligned}
& 2(n+1)(n+\alpha+\beta+1)(2 n+\alpha+\beta) P_{n+1}^{(\alpha, \beta)}(x) \\
& =\left((2 n+\alpha+\beta+1)\left(\alpha^{2}-\beta^{2}\right)+(2 n+\alpha+\beta)_{3} x\right) P_{n}^{(\alpha, \beta)}(x)- \\
& 2(n+\alpha)(n+\beta)(2 n+\alpha+\beta+2) P_{n-1}^{(\alpha, \beta)}(x),
\end{aligned}
$$

em que $(m)_{n}$ é o símbolo de Pochhammer ${ }^{1}$, que é definido por

$$
(m)_{n}=m(m+1) \cdots(m+n-1)=\frac{(m+n-1) !}{(m-1) !} .
$$

Os polinômios de Jacobi $y=P_{m}^{(\alpha, \beta)}(x)$ satisfazem a seguinte equação diferencial linear homogênea de segunda ordem:

$$
\left(1-x^{2}\right) y^{\prime \prime}+(\beta-\alpha-(\alpha+\beta+2) x) y^{\prime}+n(n+\alpha+\beta+1) y=0,
$$

em que a derivação é com respeito à $x$.

Quando $\alpha=\beta$ os polinômios de Jacobi são chamados de polinômios ultra-esféricos.

Exemplo 4.1.2 Os polinômios (ultra-esféricos) de Gegenbauer ${ }^{2},\left\{Q_{n}^{(\lambda)}(x)\right\}$ são definidos para um inteiro $n$ e são dados em termos de polinômios de Jacobi (veja o Exemplo 4.1.1) da seguinte forma

$$
Q_{n}^{(\lambda)}(x)=\frac{\Gamma\left(\lambda+\frac{1}{2}\right)}{\Gamma(2 \lambda)} \frac{\Gamma(n+2 \lambda)}{\Gamma\left(n+\lambda+\frac{1}{2}\right)} P_{n}^{(\lambda-1 / 2, \lambda-1 / 2)}(x)
$$

em que $\Gamma(z)$ indica a função Gamma complexa com parte real positiva, que é definida por

$$
\Gamma(z)=\int_{0}^{\infty} e^{-t} t^{z-1} d t
$$

$\left\{Q_{n}^{\lambda}(x)\right\}$ é uma família de polinômios ortogonais com respeito a $\left(1-x^{2}\right)^{\lambda-1 / 2}$ em $(-1,1)$.

\footnotetext{
${ }^{1}$ Leo August Pochhammer (1841 - 1920) foi um matemático alemão conhecido por seu trabalho em funções especiais.

${ }^{2}$ Leopold Bernhard Gegenbauer (1849- 1903) foi um matemático austríaco com contribuições significativas em álgebra.
} 
Estes polinômios satisfazem a seguinte equação diferencial ordinária de segunda ordem

$$
\left(1-x^{2}\right) y^{\prime \prime}-(2 \lambda+1) x y^{\prime}+n(n+2 \lambda) y=0
$$

em que a derivação é com respeito a $x$. Além disso, estes polinômios também podem ser dados em termos da seguinte função geradora

$$
\frac{1}{\left(1-2 x t+t^{2}\right)^{\lambda}}=\sum_{n=0}^{\infty} Q_{n}^{(\lambda)}(x) t^{n} .
$$

Exemplo 4.1.3 Considere $a>|b|$ parâmetros reais. Escreveremos

$$
h(\theta)=\frac{a \cos (\theta)+b}{2 \sin (\theta)}
$$

e definiremos os polinômios de Pollaczek ${ }^{3} P_{n}(x ; a, b)$ como sendo dados pela seguinte função geradora

$$
\begin{aligned}
f(x, w) & =f(\cos (\theta), w)=\sum_{n=0}^{\infty} P_{n}(x ; a, b) w^{n} \\
& =\left(1-w e^{i \theta}\right)^{-\frac{1}{2}+i h(\theta)}\left(1-w e^{-i \theta}\right)^{-\frac{1}{2}-i h(\theta)}
\end{aligned}
$$

ou ainda, de forma alternativa:

$$
f(x, w)=\left(1-2 x w+w^{2}\right)^{-\frac{1}{2}} \exp \left\{(a x+b) \sum_{m=1}^{\infty} \frac{w^{m}}{m} U_{m-1}(x)\right\}
$$

em que $U_{m-1}$ denota o caso especial de polinômio de Gegenbauer com $\lambda=1$ (também conhecido como polinômio de Chebyshev ${ }^{4}$ de segundo tipo). Os polinômios de Pollaczek satisfazem a seguinte relação de recorrência.

$$
n P_{n}(x)=[(2 n-1+2 a) x+2 b] P_{n-1}(x)-(n-1) P_{n-2},
$$

para $n=2,3,4, \ldots$ e em que $P_{0}=1$ e $P_{1}=(2 a+1) x+2 b$. Note que estes polinômios, são um caso especial dos polinômios ultra-esféricos quando $a=b=0$.

Exemplo 4.1.4 Os polinômios de Laguerre ${ }^{5}\left\{L_{n}^{(\alpha)}\right\}$ são definidos para $\alpha>-1$ por

$$
\int_{0}^{\infty} e^{-x} x^{\alpha} L_{n}^{(\alpha)}(x) L_{m}^{(\alpha)}(x) d x=\Gamma\left(\alpha_{1}+1\right)\left(\begin{array}{c}
n+\alpha \\
n
\end{array}\right) \delta_{n m}
$$

para $n, m=0,1,2, \ldots$

Além disso, é necessário que o coeficiente $x^{n}$ de $L_{n}^{(\alpha)}(x)$ de grau $n$ tenha sinal $(-1)^{n}$. Temos as seguintes equações diferenciais

$$
\begin{aligned}
x y^{\prime \prime}+(\alpha+1-x) y^{\prime}+n y=0, & y=L_{n}^{(\alpha)}(x) \\
x z^{\prime \prime}+(x+1) z^{\prime}+\left(n+\frac{\alpha}{2}+1-\frac{\alpha^{2}}{4 x}\right) z=0, & z=e^{-x} x^{\alpha / 2} L_{n}^{(\alpha)}(x), \\
u^{\prime \prime}+\left(\frac{n+(\alpha+1) / 2}{x}+\frac{1-\alpha^{2}}{4 x^{2}}-\frac{1}{4}\right) u=0, & u=e^{-x / 2} x^{(\alpha+1) / 2} L_{n}^{(\alpha)}(x), \\
v^{\prime \prime}+\left(4 n+2 \alpha+2-x^{2}+\frac{\frac{1}{4}-\alpha^{2}}{x^{2}}\right) v=0, & v=e^{-x^{2} / 2} x^{\alpha+\frac{1}{2}} L_{n}^{(\alpha)}\left(x^{2}\right) .
\end{aligned}
$$

\footnotetext{
${ }^{3}$ Félix Pollaczek (1892 - 1981) foi um matemático e engenheiro austro-francês com contribuições significativas em teoria dos números, análise matemática, física matemática e teoria de probabilidades.

${ }^{4}$ Pafnuti Chebyshev (1821 - 1894) foi um matemático russo com contribuições em probabilidade e estatística, mecânica e teoria dos números.

${ }^{5}$ Edmond Nicolas Laguerre (1834 - 1886) foi um matemático francês com contribuições significativas em geometria, análise complexa e polinômios ortogonais.
} 
Uma condição necessária e suficiente para que

$$
x y^{\prime \prime}+(\alpha+1-x) y^{\prime}+\lambda y=0,
$$

tenha solução polinomial é que $\lambda=n$. Além disso, $L_{n}^{(\alpha)}(x)$ é a única solução polinomial.

\subsection{Polinômios ortogonais associados}

Considere $\left\{P_{n}(x)\right\}$ uma família de polinômios ortogonais mônicos gerada pela seguinte relação de recursividade

$$
x P_{n}(x)=P_{n+1}(x)+\alpha_{n} P_{n}(x)+\beta_{n} P_{n-1}(x)
$$

com $n>0$, um inteiro e

$$
P_{0}(x)=1, \quad P_{1}(x)=x-\alpha_{0}
$$

em que $\alpha_{n}$ é real, para $n \geq 0$ e $\beta_{n}>0$ para $n>0$.

Os polinômios associados $\left\{P_{n}(x ; c)\right\}$ de ordem $c$ de $P_{n}(x)$ são polinômios satisfazendo as equações (4.1) e, substituindo $n$ por $n+c$ dados pela seguinte condição inicial

$$
P_{0}(x ; c)=1, \quad P_{1}(x ; c)=x-\alpha_{c} .
$$

Polinômios ortogonais associados não satisfazem à mesma função de peso que o polinômio a que se associam.

Exemplo 4.2.1 Seguindo [Ism05], temos que os polinômios de Jacobi associados $P_{n}^{(\alpha, \beta)}(x ; c)$ tem a seguinte forma explícita

$$
\begin{aligned}
& P_{n}^{(\alpha, \beta)}(x ; c)=\frac{(\gamma+2 c)_{n}(\alpha+c+1)_{n}}{(\gamma+c)_{n} n !} \\
& \cdot \sum_{k=0}^{n} \frac{(-n)_{k}(n+\gamma+2 c)_{k}}{(c+1)_{k}(\alpha+c+1)_{k}}\left(\frac{1-x}{2}\right)^{k} \\
& \cdot F_{3}\left(\begin{array}{c|c}
k-n, n+\gamma+k+2 c, \alpha+c, c & 1 \\
\alpha+k+c+1, k+c+1, \gamma+2 c-1 &
\end{array}\right),
\end{aligned}
$$

em que ${ }_{4} F_{3}$ denota uma função hipergeométrica definida da seguinte forma

$$
{ }_{r} F_{S}\left(\begin{array}{c}
a_{1}, \ldots, a_{r} \\
b_{1}, \ldots, b_{s}
\end{array} \mid z\right)=\sum_{n=0}^{\infty} \frac{\left(a_{1}, \ldots, a_{r}\right)_{n}}{\left(b_{1}, \ldots, b_{s}\right)_{n}} \frac{z^{n}}{n !},
$$

em que $\left(a_{1}, \ldots, a_{m}\right)_{n}=\prod_{j=1}^{m}\left(a_{j}\right)_{n}$ (conhecido como fatorial multishifted) definido em termos de fatoriais shifted $(a)_{n}=\Gamma(a+n) / \Gamma(a)$.

Observação: Definimos como polinômios ortogonais clássicos os polinômios ortogonais que são mais comumente utilizados: polinômios de Jacobi (incluindo os casos ultra-esféricos), polinômios de Pollaczek, polinômios de Laguerre, por exemplo. Polinômios ortogonais associados não são polinômios clássicos. 


\section{3 Álgebras DJKM e polinômios ortogonais}

Para descrever as relações de comutatividade para as álgebras DJKM foi necessário definir a família de polinômios dada por (3.4):

$$
(6+2 k) P_{k}(c)=4 k c P_{k-2}(c)-2(k-3) P_{k-4}(c)
$$

em que $c=\left(a^{2}+b^{2}\right) / 2$ e $k \geq 0$. Fixe

$$
P(c, z):=\sum_{k \geq-4} P_{k}(c) z^{z+4}=\sum_{k \geq 0} P_{k-4}(c) z^{k} .
$$

Rearranjando os termos obtemos que

$$
\begin{aligned}
0= & \sum_{k \geq 0}(6+2 k) P_{k}(c) z^{k}-4 c \sum_{k \geq 0} k P_{k-2}(c) z^{k}+2 \sum_{k \geq 0}(k-3) P_{k-4}(c) z^{k} \\
= & \left(-2 z^{-4}+8 c z^{-2}-6\right) P(c, z)+\left(2 z^{-3}-4 c z^{-1}+2 z\right) \frac{\mathrm{d}}{\mathrm{d} z} P(c, z) \\
& +\left(2 z^{-4}-8 c z^{-2}\right) P_{-4}(c)-4 c P_{-3}(c) z^{-1}-2 P_{-2}(c) z^{-2}-4 P_{-1}(c) z^{-1}
\end{aligned}
$$

Portanto, $P(c, z)$ deve satisfazer a equação diferencial

$$
\frac{\mathrm{d}}{\mathrm{d} z} P(c, z)-\frac{3 z^{4}-4 z^{2}+1}{z^{5}-2 c z^{3}+z} P(c, z)=\frac{2\left(P_{-1}+c P_{-3}\right) z^{3}+P_{-2} z^{2}+\left(4 c z^{2}-1\right) P_{-4}}{z^{5}-2 c z^{3}+z} .
$$

Esta equação tem fator integrante dado por

$$
\mu(z)=\frac{1}{z \sqrt{1-2 c z^{2}+z^{4}}} .
$$

Podemos impor determinadas condições iniciais para estudar esta família de polinômios, como observado em [CFT13]. A ideia é fixar 3 parâmetros iguais a zero e outro parâmetro igual a 1 com o objetivo de alcançar equações diferenciais ordinárias de grau 4. Os casos analisados serão apresentados a seguir.

\subsubsection{Caso elíptico 1}

Considere que $P_{-3}(c)=P_{-2}(c)=P_{-1}(c)=0$ e $P_{-4}(c)=1$, então teremos a seguinte função geradora

$$
P_{-4}(c, z):=\sum_{k \geq-4} P_{-4, k}(c) z^{z+4}=\sum_{k \geq 0} P_{-4, k-4}(c) z^{k},
$$

definida em termos de uma integral elíptica

$$
P_{-4}(c, z)=z \sqrt{1-2 c z^{2}+z^{4}} \int \frac{4 c z^{2}-1}{z^{2}\left(z^{4}-2 c z^{2}+1\right)^{3 / 2}} \mathrm{~d} z .
$$

Re-indexando as famílias de polinômios $P_{-4, k}$, temos que

$$
\begin{aligned}
P_{-4}(c, z)= & z \sqrt{1-2 c z^{2}+z^{4}} \int \frac{4 c z^{2}-1}{z^{2}\left(z^{4}-2 c z^{2}+1\right)^{3 / 2}} \mathrm{~d} z=\sum_{n=0}^{\infty} P_{-4, n}(c) z^{n} \\
= & 1+z^{4}+\frac{4 c}{5} z^{6}+\frac{1}{35}\left(32 c^{2}-5\right) z^{8}+\frac{16}{105} c\left(8 c^{2}-3\right) z^{10} \\
& -\frac{\left(2048 c^{4}-1248 c^{2}+75\right)}{1155} z^{12}+O\left(z^{14}\right) .
\end{aligned}
$$


Isso significa que agora $P_{-4,0}(c)=1, P_{-4,1}(c)=P_{-4,2}(c)=P_{-4,3}(c)=0$. Além de $P_{-4,0}(c)$, os primeiros polinômios não nulos em $c$ são

$$
\begin{aligned}
& P_{-4,4}(c)=1, P_{-4,6}(c)=\frac{4 c}{5}, P_{-4,8}(c)=\frac{\left(32 c^{2}-5\right)}{35}, \\
& P_{-4,10}(c)=\frac{16}{105} c\left(8 c^{2}-3\right), P_{-4,12}(c)=-\frac{\left(2048 c^{4}-1248 c^{2}+75\right)}{1155} .
\end{aligned}
$$

Note que, partindo da função geradora,

$$
\begin{aligned}
P_{-4}(c, z): & \sum_{k \geq-4} P_{-4, k}(c) z^{z+4}=\sum_{k \geq 0} P_{-4, k-4}(c) z^{k} \\
& =z \sqrt{1-2 c z^{2}+z^{4}}\left(\sum_{n=0}^{\infty} \frac{4 c Q_{n}^{(3 / 2)}(c)}{2 n+1} z^{2 n+1}-\sum_{n=0}^{\infty} \frac{Q_{n}^{(3 / 2)}(c)}{2 n-1} z^{2 n-1}\right),
\end{aligned}
$$

em que $Q_{n}^{(\lambda)}(c)$ é o polinômio de Gegenbauer de ordem $n$ (veja o Exemplo 4.1.2).

Teorema 4.3.1 ([CFT13], Teorema 3.1.1) Os polinômios $P_{-4, k}$ satisfazem a seguinte equação diferencial:

$$
\begin{aligned}
& 16\left(c^{2}-1\right)^{2} P_{n}^{(i v)}+160 c\left(c^{2}-1\right) P_{n}^{\prime \prime \prime}-8\left(c^{2}\left(n^{2}-4 n-46\right)-n^{2}+4 n+22\right) P_{n}^{\prime \prime} \\
& -24 c\left(n^{2}-4 n-6\right) P_{n}^{\prime}+(n-4)^{2} n^{2} P_{n}=0 .
\end{aligned}
$$

Teorema 4.3.2 ([CFT13], Corolário 4.0.2) Os polinômios $P_{-4, n}$ são ortogonais não-clássi$\cos$.

\subsubsection{Caso elíptico 2}

Considere que $P_{-4}(c)=P_{-3}(c)=P_{-1}(c)=0$ e $P_{-2}(c)=1$, então teremos a seguinte função geradora definida em termos de uma integral elíptica

$$
P_{-2}(c, z)=z \sqrt{1-2 c z^{2}+z^{4}} \int \frac{1}{\left(z^{4}-2 c z^{2}+1\right)^{3 / 2}} \mathrm{~d} z .
$$

Re-indexando as famílias de polinômios $P_{-4, k}$, temos que

$$
P_{-2}(c, z)=z \sqrt{1-2 c z^{2}+z^{4}} \int \frac{1}{\left(z^{4}-2 c z^{2}+1\right)^{3 / 2}} \mathrm{~d} z=\sum_{n=0}^{\infty} P_{-2, n}(c) z^{n} .
$$

Isso significa que agora $P_{-2,2}(c)=1, P_{-2,3}(c)=P_{-2,1}(c)=P_{-2,0}(c)=0$. Além de $P_{-2,3}(c)$, os primeiros polinômios não nulos em $c$ são

$$
\begin{aligned}
& P_{-2,2}(c)=1, P_{-2,6}(c)=\frac{1}{5}, P_{-2,8}(c)=\frac{8 c}{35}, \\
& P_{-2,10}(c)=\frac{\left(-7+32 c^{2}\right)}{105}, P_{-2,12}(c)=\frac{8 c\left(-29+64 c^{2}\right)}{1155},
\end{aligned}
$$

então

$$
P_{-2}(c, z)=z^{2}+\frac{1}{5} z^{6}+\frac{8 c}{35} z^{8}+\frac{\left(-7+32 c^{2}\right)}{105} z^{10}-\frac{8 c\left(-29+64 c^{2}\right)}{1155} z^{12}+O\left(z^{14}\right) .
$$


Teorema 4.3.3 ([CFT13], Teorema 3.2.1) Os polinômios $P_{-2, k}$ satisfazem a seguinte equação diferencial:

$$
\begin{aligned}
& 16\left(c^{2}-1\right)^{2} P_{n}^{(i v)}+160 c\left(c^{2}-1\right) P_{n}^{\prime \prime \prime}-8\left(c^{2}\left(n^{2}-4 n-42\right)-n^{2}+4 n+18\right) P_{n}^{\prime \prime} \\
& -24 c\left(n^{2}-4 n-2\right) P_{n}^{\prime}+(n-6)(n-2)^{2}(n+2) P_{n}=0 .
\end{aligned}
$$

Teorema 4.3.4 ([CFT13], Teorema 5.0.5) Os polinômios $P_{-2, k}$ são ortogonais com respeito a alguma função peso.

\section{4 Álgebras de Krichever-Novikov superelípticas e polinô- mios ortogonais}

Para descrever as álgebras superelípticas foi necessário considerar a família de polinômios dada por (3.18):

$$
t^{n} u^{l} d t \equiv \sum_{k=0}^{D-1}\left(-a_{k}\left(\frac{k l+(-D+1+n+k) m}{D l+(1+n) m}\right)\right) t^{-D+n+k} u^{l} d t(\bmod d R) .
$$

De forma a estudar uma variação do caso apresentado na subseção anterior, iniciaremos considerando a curva dada por $u^{m}=t^{4}-2 c t^{2}+1$ em que $c \in \mathbb{C} \backslash\{-1,1\}$ e $m \geq 2$. Fixando $l=1$ e $\operatorname{com} a_{1}=a_{3}=0, a_{0}=a_{4}=1$ e $a_{2}=-2 c$, teremos

$$
2 c((i+2) m+2) \overline{t^{i+1} \mathrm{udt}}=i m \overline{t^{i-1} \mathrm{udt}}+(-(i+4) m-4) \overline{t^{i+3} \mathrm{udt}}
$$

e se fizermos $n=i+3$ obtemos que

$$
(m n+m+4) \overline{t^{n} \mathrm{udt}}=2 c(m(n-1)+2) \overline{t^{n-2} \mathrm{udt}}-m(n-3) \overline{t^{n-4} \mathrm{udt}} .
$$

Considere $P_{k}:=P_{k}(c)$ a família de polinômios em $c$ satisfazendo a seguinte relação de recursividade

$$
(m n+m+4) P_{n}(c)=2 c(m(n-1)+2) P_{n-2}(c)-m(n-3) P_{n-4}(c)
$$

para $n \geq 0$. Além disso, definamos

$$
P(c, z):=\sum_{n \geq-4} P_{n}(c) z^{n+4}=\sum_{n \geq 0} P_{n-4}(c) z^{n},
$$

e então após o rearranjar os termos, teremos que

$$
\begin{aligned}
0= & \sum_{n=0}^{\infty} m(n-3) z^{n} P_{n-4}(c)-(2 c) \sum_{n=0}^{\infty}(m n-m+2) z^{n} P_{n-2}(c) \\
& +\sum_{n=0}^{\infty}(m n+m+4) z^{n} P_{n}(c) \\
= & \left(2 c(3 m-2) z^{2}-3 m z^{4}-3 m+4\right) P(c, z)\left(z^{-4}\right)+P_{-4}\left(2 c(2-3 m) z^{2}+3 m-4\right) z^{-4} \\
& m\left(-2 c z^{2}+z^{4}+1\right) \frac{\mathrm{d}}{\mathrm{dz}} P(c, z) z^{-3}+\frac{2 P_{-3}\left(-2 c(m-1) z^{2}+m-2\right)}{z^{3}} \\
& +\frac{(m-4) P_{-2}}{z^{2}}-\frac{4 P_{-1}}{z} .
\end{aligned}
$$


Portanto, $P(c, z)$ deve satisfazer a seguinte equação diferencial

$\frac{\mathrm{d}}{\mathrm{d} z} P(c, z)-\frac{-6 c m z^{2}+4 c z^{2}+3 m z^{4}+3 m-4}{m z\left(-2 c z^{2}+z^{4}+1\right)} P(c, z)=$

$\frac{-\left(2 c(2-3 m) z^{2}+3 m-4\right) z^{-4} P_{-4}-2\left(-2 c(m-1) z^{2}+m-2\right) z^{-3} P_{-3}-(m-4) z^{-2} P_{-2}+4 z^{-1} P_{-1}}{m z^{-3}\left(-2 c z^{2}+z^{4}+1\right)}$

Esta equação possui fator integrante dado por

$$
\begin{aligned}
\mu(z) & =\exp \left(\int-\frac{-6 c m z^{2}+4 c z^{2}+3 m z^{4}+3 m-4}{m z\left(-2 c z^{2}+z^{4}+1\right)} \mathrm{dz}\right) \\
& =\exp \left(\frac{(4-3 m) \log (z)}{m}-\frac{\log \left(-2 c z^{2}+z^{4}+1\right)}{m}\right) \\
& =\frac{1}{z^{3-4 / m}\left(-2 c z^{2}+z^{4}+1\right)^{1 / m}} .
\end{aligned}
$$

A seguir analisaremos o que obtém-se após impor algumas condições iniciais. Em especial, trataremos primeiramente o caso em que $m=3$ com a expectativa de, futuramente, obter resultados similares para o caso genérico.

\subsubsection{Caso 1}

Considere que $P_{-4}=P_{-3}=P_{-2}=0$ e $P_{-1}=1$ e a seguinte função geradora.

$$
P_{-1}(c, z):=\sum_{k \geq-4} P_{-1, k}(c) z^{z+4}=\sum_{k \geq 0} P_{-1, k-4}(c) z^{k}
$$

Esta função geradora deve ser definida em termos da seguinte integral.

$$
P_{-1}(c, z):=z^{3-\frac{4}{m}}\left(-2 c z^{2}+z^{4}+1\right)^{1 / m} \int \frac{4 z^{\frac{4}{m}-1}}{m\left(-2 c z^{2}+z^{4}+1\right)^{\frac{1}{m}+1}} \mathrm{~d} z .
$$

Em particular, se $m=3$ teremos que

$$
P_{-1}(c, z):=z^{5 / 3} \sqrt[3]{-2 c z^{2}+z^{4}+1} \int \frac{4 \sqrt[3]{z}}{3\left(-2 c z^{2}+z^{4}+1\right)^{4 / 3}} d z
$$

\subsubsection{Caso 2}

Considere que $P_{-4}=P_{-2}=P_{-1}=0$ e $P_{-3}=1$ e a seguinte função geradora.

$$
P_{-3}(c, z):=\sum_{k \geq-4} P_{-3, k}(c) z^{z+4}=\sum_{k \geq 0} P_{-3, k-4}(c) z^{k} .
$$

Esta função geradora deve ser definida em termos da seguinte integral.

$$
P_{-3}(c, z):=z^{3-\frac{4}{m}}\left(-2 c z^{2}+z^{4}+1\right)^{1 / m} \int \frac{z^{\frac{4}{m}-3}\left(4 z^{2}(c(m-1)+1)-2 m+4\right)}{m\left(-2 c z^{2}+z^{4}+1\right)^{\frac{1}{m}+1}} \mathrm{dz} .
$$

Em particular, se $m=3$ teremos que

$$
P_{-3}(c, z):=z^{5 / 3} \sqrt[3]{-2 c z^{2}+z^{4}+1} \int \frac{2\left(4 c z^{2}-1\right)}{3 z^{5 / 3}\left(-2 c z^{2}+z^{4}+1\right)^{4 / 3}} d z
$$




\subsubsection{Caso 3}

Considere que $P_{-4}=P_{-3}=P_{-1}=0$ e $P_{-2}=1$ e a seguinte função geradora.

$$
P_{-2}(c, z):=\sum_{k \geq-4} P_{-2, k}(c) z^{z+4}=\sum_{k \geq 0} P_{-2, k-4}(c) z^{k} .
$$

Esta função geradora deve ser definida em termos da seguinte integral.

$$
P_{-2}(c, z):=z^{3-\frac{4}{m}}\left(-2 c z^{2}+z^{4}+1\right)^{1 / m} \int \frac{(4-m) z^{\frac{4}{m}-2}}{m\left(-2 c z^{2}+z^{4}+1\right)^{\frac{1}{m}+1}} \mathrm{dz} .
$$

Em particular, se $m=3$ teremos que

$$
P_{-2}(c, z):=z^{5 / 3} \sqrt[3]{-2 c z^{2}+z^{4}+1} \int \frac{1}{3 z^{2 / 3}\left(-2 c z^{2}+z^{4}+1\right)^{4 / 3}} \mathrm{dz}
$$

então

$$
\begin{aligned}
P_{-2}(c, z)= & \left(28\left(c^{2}-1\right)\right)^{-1} \\
& \left(7\left(2 c^{2}-3\right) z^{2} R(c, z) F_{1}\left(\frac{1}{6} ; \frac{1}{3}, \frac{1}{3} ; \frac{7}{6} ;\left(c+\sqrt{c^{2}-1}\right) z^{2}, \frac{z^{2}}{c+\sqrt{c^{2}-1}}\right)\right. \\
& +3 c z^{4} R(c, z) F_{1}\left(\frac{7}{6} ; \frac{1}{3}, \frac{1}{3} ; \frac{13}{6} ;\left(c+\sqrt{c^{2}-1}\right) z^{2}, \frac{z^{2}}{c+\sqrt{c^{2}-1}}\right) \\
& \left.+c_{1} 28\left(c^{2}-1\right) \sum_{n=0}^{\infty} z^{2 n+\frac{5}{3}} Q_{n}^{\left(-\frac{1}{3}\right)}(c)-7 z^{2}\left(c\left(z^{2}-2 c\right)+1\right)\right),
\end{aligned}
$$

aqui $Q_{-1 / 2}^{(\lambda)}(c)$ denota o polinômio de Gegenbauer com $\lambda=-1 / 2, c_{1}$ é uma constante arbitrária, $R(c, z)$ é um "polinômio"6 em $c$ e $z$ definido da seguinte maneira,

$$
R(c, z)=\left(\left(\left(\sqrt{c^{2}-1}-c\right) z^{2}+1\right)\left(1-\left(\sqrt{c^{2}-1}+c\right) z^{2}\right)\right)^{1 / 3}
$$

e $F_{1}(a ; b ; c ; d)$ denota a função hipergeométrica de Appell ${ }^{7}$. A função $F_{1}$ é definida para $|x|<1$ e $|y|<1$ de forma que

$$
F_{1}\left(a, b_{1}, b_{2} ; c, x, y\right)=\sum_{m, n=0}^{\infty} \frac{(a)_{m+n}\left(b_{1}\right)_{m}\left(b_{2}\right)_{n}}{(c)_{m+n} m ! n !} x^{m} y^{n}
$$

\subsubsection{Caso 4}

Considere que $P_{-3}=P_{-2}=P_{-1}=0$ e $P_{-4}=1$ e a seguinte função geradora.

$$
P_{-4}(c, z):=\sum_{k \geq-4} P_{-4, k}(c) z^{z+4}=\sum_{k \geq 0} P_{-4, k-4}(c) z^{k} .
$$

Esta função geradora deve ser definida em termos da seguinte integral.

$$
P_{-4}(c, z):=z^{3-\frac{4}{m}}\left(-2 c z^{2}+z^{4}+1\right)^{1 / m} \int \frac{\left(2 c(3 m-2) z^{2}-3 m+4\right) z^{\frac{4}{m}-4}}{m\left(-2 c z^{2}+z^{4}+1\right)^{\frac{1}{m}+1}} \mathrm{dz} .
$$

\footnotetext{
6à rigor não é um polinômio pois aparecem raízes terceiras

7 Paul Appell (1855 - 1930) foi um matemático francês e reitor da Universidade de Paris
} 
Em particular, se $m=3$ teremos que

$$
P_{-4}(c, z):=z^{5 / 3} \sqrt[3]{-2 c z^{2}+z^{4}+1} \int \frac{14 c z^{2}-5}{3 z^{8 / 3}\left(-2 c z^{2}+z^{4}+1\right)^{4 / 3}} \mathrm{dz},
$$

então

$$
\begin{aligned}
P_{-4}(c, z)= & \left(28\left(c^{2}-1\right)\right)^{-1} \\
& \left(-63 c z^{2} R(c, z) F_{1}\left(\frac{1}{6} ; \frac{1}{3}, \frac{1}{3} ; \frac{7}{6} ;\left(c+\sqrt{c^{2}-1}\right) z^{2}, \frac{z^{2}}{c+\sqrt{c^{2}-1}}\right)\right. \\
& +27 z^{4} R(c, z) F_{1}\left(\frac{7}{6} ; \frac{1}{3}, \frac{1}{3} ; \frac{13}{6} ;\left(c+\sqrt{c^{2}-1}\right) z^{2}, \frac{z^{2}}{c+\sqrt{c^{2}-1}}\right) \\
& \left.+c_{1} 28\left(c^{2}-1\right) \sum_{n=0}^{\infty} z^{2 n+\frac{5}{3}} Q_{n}^{\left(-\frac{1}{3}\right)}(c)+7\left(4 c^{2}+9 c z^{2}-9 z^{4}-4\right)\right) .
\end{aligned}
$$

\subsubsection{Equações diferenciais para os Casos 1 e 2}

Nesta seção continuaremos o estudo do caso particular $m=3$, desta forma, faz sentido observar que, $\operatorname{com} m=3$ teremos a seguinte relação de recursividade.

$$
(7+3 n) P_{n}(c)=-3(n-3) P_{n-4}(c)+2 c(2+3(-1+n)) P_{n-2}(c) .
$$

Para encontrar uma equação diferencial linear de ordem 4 que os polinômios do tipo $P_{-1, n}$ e $P_{-3, n}$ satisfazem utiliza-se a mesma estratégia encontrada no artigo [CFT13].

\section{Caso 1}

Reindexaremos os polinômios da seguinte forma:

$$
\begin{aligned}
P_{-1}(c, z)= & \left(\frac{4}{3}\right) z^{5 / 3}\left(-2 c z^{2}+z^{4}+1\right)^{1 / 3} \int \frac{z^{1 / 3}}{\left(-2 c z^{2}+z^{4}+1\right)^{4 / 3}} \mathrm{~d} z=\sum_{n=0}^{\infty} P_{n} z^{n} \\
= & z^{3}+\frac{2 c z^{5}}{5}+\frac{2 c^{2} z^{7}}{5}+\frac{1}{55}(2 c)\left(14 c^{2}-3\right) z^{9}+\frac{1}{55}\left(2 c^{2}\right)\left(20 c^{2}-9\right) z^{11} \\
& +\frac{1}{935}(2 c)\left(520 c^{4}-360 c^{2}+27\right) z^{13}+O\left(z^{14}\right)
\end{aligned}
$$

Isto significa que de agora em diante, $P_{-1,3}=1, P_{-1,0}=P_{-1,1}=P_{-1,2}=0$. Além de $P_{-1,3}$, os primeiros polinômios não nulos desta família são

$$
\begin{aligned}
P_{-1,5}(c) & =\frac{2 c}{5}, & P_{-1,7}(c)=\frac{2 c^{2}}{5} \\
P_{-1,9}(c) & =\frac{2}{55} c\left(14 c^{2}-3\right), & P_{-1,11}(c)=\frac{2}{55} c^{2}\left(20 c^{2}-9\right) \\
\text { e } P_{-1,13}(c) & =\frac{2}{935} c\left(520 c^{4}-360 c^{2}+27\right) . &
\end{aligned}
$$

O objetivo é encontrar famílias de equações diferenciais lineares em $c$ que estes polinômios satisfazem. Iniciaremos o estudo, considerando a seguinte função geradora

$$
\begin{aligned}
P_{-1}(c, z) & =\left(\frac{4}{3}\right) z^{5 / 3}\left(-2 c z^{2}+z^{4}+1\right)^{1 / 3} \int \frac{z^{1 / 3}}{\left(-2 c z^{2}+z^{4}+1\right)^{4 / 3}} \mathrm{~d} z \\
& =\left(\frac{4}{3}\right) z^{5 / 3}\left(-2 c z^{2}+z^{4}+1\right)^{1 / 3} \sum_{n=0}^{\infty} \frac{Q_{n}^{(4 / 3)}(c)}{2 n+1 / 3} z^{2 n+1 / 3},
\end{aligned}
$$


em que $Q_{n}^{(\lambda)}(c)$ é o polinômio de Gegenbauer de ordem $n$. Estes polinômios satisfazem a seguinte equação diferencial linear de segunda ordem:

$$
\left(1-c^{2}\right)\left(Q_{n}^{(4 / 3)}(c)\right)^{\prime \prime}-\frac{11}{3} c\left(Q_{n}^{(4 / 3)}(c)\right)^{\prime}+\left(n+\frac{8}{3}\right) n Q_{n}^{(4 / 3)}(c)=0
$$

Reescrevendo a equação expandida de $P_{-1}(c, z)$, obtemos que

$$
\left(\frac{3}{4}\right) z^{-5 / 3}\left(-2 c z^{2}+z^{4}+1\right)^{-1 / 3} P_{-1}(c, z)=\sum_{n=0}^{\infty} \frac{Q_{n}^{(4 / 3)}(c)}{2 n+1 / 3} z^{2 n+1 / 3},
$$

e aplicando o operador diferencial $L:=\left(1-c^{2}\right) \frac{d^{2}}{d c^{2}}-\frac{11}{3} c \frac{d}{d c}$ no lado direito da equação acima, obtemos que

$$
\begin{aligned}
L\left(Q_{n}^{(4 / 3)}(c)\right) & =\left(1-c^{2}\right)\left(Q_{n}^{(4 / 3)}(c)\right)^{\prime \prime}-\frac{11}{3} c\left(Q_{n}^{(4 / 3)}(c)\right)^{\prime} \\
& =-(n+8 / 3) n Q_{n}^{(4 / 3)}(c) .
\end{aligned}
$$

Chegamos a

$$
\begin{aligned}
L\left(\sum_{n=0}^{\infty} \frac{Q_{n}^{(4 / 3)}(c)}{2 n+1 / 3} z^{2 n+1 / 3}\right)= & \sum_{n=0}^{\infty}-\frac{\left(n(3 n+8) Q_{n}^{(4 / 3)}(c)\right)}{3(2 n+1 / 3)} z^{2 n+1 / 3} \\
= & \sum_{n=0}^{\infty}\left(-\frac{n}{2}+\frac{5}{4(6 n+1)}-\frac{5}{4}\right) Q_{n}^{(4 / 3)}(c) z^{2 n+1 / 3} \\
= & \frac{1}{2} \sum_{n=0}^{\infty}\left(-n+\frac{5}{2(6 n+1)}-\frac{5}{2}\right) Q_{n}^{(4 / 3)}(c) z^{2 n+1 / 3} \\
= & \frac{-5}{4} \sum_{n=0}^{\infty} Q_{n}^{(4 / 3)}(c) z^{2 n+1 / 3}-\frac{1}{2} \sum_{n=0}^{\infty} n Q_{n}^{(4 / 3)}(c) z^{2 n+1 / 3} \\
& +\frac{5}{12} \sum_{n=0}^{\infty} \frac{Q_{n}^{(4 / 3)}(c)}{2 n+1 / 3} z^{2 n+1 / 3} \\
= & \frac{-5}{4} z^{1 / 3}\left(\sum_{n=0}^{\infty} Q_{n}^{(4 / 3)}(c) z^{2 n}\right)-\frac{1}{2} z^{4 / 3}\left(\sum_{n=0}^{\infty} n Q_{n}^{(4 / 3)}(c) z^{2 n-1}\right) \\
& +\frac{5}{12} \sum_{n=0}^{\infty} \frac{Q_{n}^{(4 / 3)}(c)}{2 n+1 / 3} z^{2 n+1 / 3} \\
= & -\frac{5 z^{1 / 3}}{4\left(-2 c z^{2}+z^{4}+1\right)^{4 / 3}}+\frac{5}{12} \int \frac{1}{z^{2 / 3}\left(-2 c z^{2}+z^{4}+1\right)^{4 / 3}} \mathrm{~d} z \\
& -\frac{1}{4} z^{4 / 3} \frac{\partial}{\partial z}\left(\frac{1}{\left(-2 c z^{2}+z^{4}+1\right)^{4 / 3}}\right) .
\end{aligned}
$$

Então, o membro direito da igualdade (4.4) se torna

$$
\frac{5}{4\left(z^{5 / 3}\left(-2 c z^{2}+z^{4}+1\right)^{1 / 3}\right)} P_{-4}+\frac{z^{1 / 3}\left(14 c z^{2}+z^{4}-15\right)}{12\left(-2 c z^{2}+z^{4}+1\right)^{7 / 3}} .
$$


Aplicando o operador diferencial $L$ ao membro esquerdo de (4.4), obtemos

$$
\begin{aligned}
L\left(\frac{3 P_{-2}(c, z)}{4 z^{5 / 3}\left(-2 c z^{2}+z^{4}+1\right)^{1 / 3}}\right) & =\left(\frac{z^{1 / 3}\left(14 c^{2} z^{2}-11 c\left(z^{4}+1\right)+8 z^{2}\right)}{6\left(-2 c z^{2}+z^{4}+1\right)^{7 / 3}}\right) P_{-2}(c, z) \\
+ & \left(\frac{18 c^{2} z^{2}-11 c\left(z^{4}+1\right)+4 z^{2}}{4 z^{5 / 3}\left(-2 c z^{2}+z^{4}+1\right)^{4 / 3}}\right) P_{-2}^{\prime}(c, z) \\
+ & \left(-\frac{3\left(c^{2}-1\right)}{4 z^{5 / 3}\left(-2 c z^{2}+z^{4}+1\right)^{1 / 3}}\right) P_{-2}^{\prime \prime}(c, z) .
\end{aligned}
$$

Consequentemente

$$
\begin{aligned}
& \left(\frac{z^{1 / 3}\left(14 c^{2} z^{2}-11 c\left(z^{4}+1\right)+8 z^{2}\right)}{6\left(-2 c z^{2}+z^{4}+1\right)^{7 / 3}}\right) P_{-2}(c, z)+ \\
& \left(\frac{18 c^{2} z^{2}-11 c\left(z^{4}+1\right)+4 z^{2}}{4 z^{5 / 3}\left(-2 c z^{2}+z^{4}+1\right)^{4 / 3}}\right) P_{-2}^{\prime}(c, z)-\left(\frac{3\left(c^{2}-1\right)}{4 z^{5 / 3}\left(-2 c z^{2}+z^{4}+1\right)^{1 / 3}}\right) P_{-2}^{\prime \prime}(c, z) \\
= & \left(\frac{5}{4 z^{5 / 3}\left(-2 c z^{2}+z^{4}+1\right)^{1 / 3}}\right) P_{-2}(c, z)+\frac{z^{1 / 3}\left(14 c z^{2}+z^{4}-15\right)}{12\left(-2 c z^{2}+z^{4}+1\right)^{7 / 3}},
\end{aligned}
$$

que nos dá que

$$
\begin{aligned}
z^{2}\left(14 c z^{2}+z^{4}-15\right)= & \left(-32 c^{2} z^{4}+38 c\left(z^{6}+z^{2}\right)-15 z^{8}-14 z^{4}-15\right) P_{-2}(c, z) \\
& +3\left(18 c^{2} z^{2}-11 c\left(z^{4}+1\right)+4 z^{2}\right)\left(-2 c z^{2}+z^{4}+1\right) P_{-2}^{\prime}(c, z) \\
& -9\left(c^{2}-1\right)\left(-2 c z^{2}+z^{4}+1\right)^{2} P_{-2}^{\prime \prime}(c, z) .
\end{aligned}
$$

Expandindo esta fórmula detalhadamente e denotando $P_{-1, k}$ por $P_{-1}$ obtemos que

$$
\begin{aligned}
0= & -15 P_{n-8}+38 c P_{n-6}+\left(-32 c^{2}-14\right) P_{n-4}+38 c P_{n-2}-15 P_{n} \\
& -33 c P_{n-8}^{\prime}+\left(-108 c^{3}-90 c\right) P_{n-4}^{\prime}+\left(120 c^{2}+12\right) P_{n-6}^{\prime} \\
& +\left(120 c^{2}+12\right) P_{n-2}^{\prime}-33 c P_{n}^{\prime} \\
& +9\left(1-c^{2}\right)\left(P_{n-8}^{\prime \prime}+2\left(2 c^{2}+1\right) P_{n-4}^{\prime \prime}-4 c P_{n-6}^{\prime \prime}-4 c P_{n-2}^{\prime \prime}+P_{n}^{\prime \prime}\right) .
\end{aligned}
$$

Agora, mudaremos $n$ para $n+4$ e diferenciaremos as relações de recursividade dadas por (4.2),

$$
0=2 c(3 n-1) P_{n-2}+3(3-n) P_{n-4}-(3 n+7) P_{n}
$$

para obter que

$$
\begin{gathered}
0=2 c(3 n+11) P_{n+2}-3(n+1) P_{n}-(3 n+19) P_{n+4} \\
0=2 c(3 n+11) P_{n+2}^{\prime}-3(n+1) P_{n}^{\prime}+(-3 n-19) P_{n+4}^{\prime}+(6 n+22) P_{n+2},
\end{gathered}
$$

e

$$
0=2 c(3 n+11) P_{n+2}^{\prime \prime}+4(3 n+11) P_{n+2}^{\prime}-3(n+1) P_{n}^{\prime \prime}-(3 n+19) P_{n+4}^{\prime \prime} .
$$

Fazendo com que $n=n-8$ na última equação, teremos que

$$
0=2 c(3 n-13) P_{n-6}^{\prime \prime}+4(3 n-13) P_{n-6}^{\prime}-3(n-7) P_{n-8}^{\prime \prime}+(5-3 n) P_{n-4}^{\prime \prime} .
$$


Multiplicando a equação $(4.5)$ por $(-7+n)$ e adicionando-a à $3\left(1-c^{2}\right)$ multiplicado pela equação acima, obtemos que

$$
\begin{aligned}
0= & -15(n-7) P_{n-8}-33 c(n-7) P_{n-8}^{\prime} \\
& +38 c(n-7) P_{n-6}+12\left(c^{2}(7 n-57)+4(n-5)\right) P_{n-6}^{\prime}+6 c\left(c^{2}-1\right)(3 n-29) P_{n-6}^{\prime \prime} \\
& -2\left(16 c^{2}+7\right)(n-7) P_{n-4}-18 c\left(6 c^{2}+5\right)(n-7) P_{n-4}^{\prime} \\
& -3\left(c^{2}-1\right)\left(12 c^{2}(n-7)+3 n-37\right) P_{n-4}^{\prime \prime} \\
& +38 c(n-7) P_{n-2}+12\left(10 c^{2}+1\right)(n-7) P_{n-2}^{\prime}+36 c\left(c^{2}-1\right)(n-7) P_{n-2}^{\prime \prime} \\
& -15(n-7) P_{n}-33 c(n-7) P_{n}^{\prime}-9\left(c^{2}-1\right)(n-7) P_{n}^{\prime \prime} .
\end{aligned}
$$

Fazendo com que $n=n-8$ em (4.7), obtemos que

$$
0=2 c(3 n-13) P_{n-6}^{\prime}-3(n-7) P_{n-8}^{\prime}+(5-3 n) P_{n-4}^{\prime}+(6 n-26) P_{n-6} .
$$

Multiplicando esta equação por $(-11 c)$ e adicionando-a à última equação, teremos que

$$
\begin{aligned}
0= & -15(n-7) P_{n-8} \\
& +4 c(5-7 n) P_{n-6}+\left(2 c^{2}(9 n-199)+48(n-5)\right) P_{n-6}^{\prime}+6 c\left(c^{2}-1\right)(3 n-29) P_{n-6}^{\prime \prime} \\
& -2\left(16 c^{2}+7\right)(n-7) P_{n-4}+c\left(-108 c^{2}(n-7)-57 n+575\right) P_{n-4}^{\prime} \\
& -3\left(c^{2}-1\right)\left(12 c^{2}(n-7)+3 n-37\right) P_{n-4}^{\prime \prime} \\
& +38 c(n-7) P_{n-2}+12\left(10 c^{2}+1\right)(n-7) P_{n-2}^{\prime}+36 c\left(c^{2}-1\right)(n-7) P_{n-2}^{\prime \prime} \\
& -15(n-7) P_{n}-33 c(n-7) P_{n}^{\prime}-9\left(c^{2}-1\right)(n-7) P_{n}^{\prime \prime} .
\end{aligned}
$$

Fazendo com que $n=n-8$ em (4.6), obtemos que

$$
0=2 c(3 n-13) P_{n-6}-3(n-7) P_{n-8}+(5-3 n) P_{n-4} .
$$

Finalmente, se multiplicarmos esta última equação por $(-5)$ e adicionando-a à equação anterior, teremos que

$$
\begin{aligned}
0= & \left(2 c^{2}(9 n-199)+48(n-5)\right) P_{n-6}^{\prime}+6 c\left(c^{2}-1\right)(3 n-29) P_{n-6}^{\prime \prime} \\
& +\left(-32 c^{2}(n-7)+n+73\right) P_{n-4}+c\left(-108 c^{2}(n-7)-57 n+575\right) P_{n-4}^{\prime} \\
& -3\left(c^{2}-1\right)\left(12 c^{2}(n-7)+3 n-37\right) P_{n-4}^{\prime \prime} \\
& +2 c(75-29 n) P_{n-6}+38 c(n-7) P_{n-2}+12\left(10 c^{2}+1\right)(n-7) P_{n-2}^{\prime} \\
& +36 c\left(c^{2}-1\right)(n-7) P_{n-2}^{\prime \prime} \\
& -9\left(c^{2}-1\right)(n-7) P_{n}^{\prime \prime}-33 c(n-7) P_{n}^{\prime}-15(n-7) P_{n} .
\end{aligned}
$$

Esta é uma equação sem $P_{n-8}$. Agora nós faremos procedimento similar para eliminar os termos do tipo $P_{n-6}$. Fazendo com que $n=n-6$ em (4.8), teremos que

$$
0=2 c(3 n-13) P_{n-6}^{\prime}-3(n-7) P_{n-8}^{\prime}+(5-3 n) P_{n-4}^{\prime}+(6 n-26) P_{n-6} .
$$

Multiplicando esta equação por $\left(2 c\left(c^{2}-1\right)(3 n-29)\right)$ e adicionando-a à $(-5+n)$ multiplicado pela equação anterior, obtemos que

$$
\begin{aligned}
0= & 2 c(n-5)(75-29 n) P_{n-6}+(n-5)\left(2 c^{2}(9 n-199)+48(n-5)\right) P_{n-6}^{\prime} \\
& +(n-5)\left(-32 c^{2}(n-7)+n+73\right) P_{n-4} \\
& -c\left(4 c^{2}(9(n-12) n+539)+n(129 n-1724)+4499\right) P_{n-4}^{\prime} \\
& -\left(c^{2}-1\right)\left(448 c^{2}+3(n-5)(3 n-37)\right) P_{n-4}^{\prime \prime} \\
& +38 c(n-7)(n-5) P_{n-2}+12\left(10 c^{2}+1\right)(n-7)(n-5) P_{n-2}^{\prime} \\
& +2 c\left(c^{2}-1\right)(3 n(3 n-44)+659) P_{n-2}^{\prime \prime} \\
& -15(n-7)(n-5) P_{n}-33 c(n-7)(n-5) P_{n}^{\prime}-9\left(c^{2}-1\right)(n-7)(n-5) P_{n}^{\prime \prime} .
\end{aligned}
$$


De (4.7), $\operatorname{com} n=n-6$ temos que

$$
0=2 c(3 n-7) P_{n-4}^{\prime}-3(n-5) P_{n-6}^{\prime}+(-3 n-1) P_{n-2}^{\prime}+(6 n-14) P_{n-4} .
$$

Multiplicando esta equação por $\left(2 c^{2}(9 n-199)+48(n-5)\right)$ e adicionando-a à equação anterior multiplicada por 3 , obtemos que

$$
\begin{aligned}
0= & +6 c(n-5)(75-29 n) P_{n-6} \\
& +114 c(n-7)(n-5) P_{n-2} \\
& +2\left(c^{2}(3 n(51 n-524)+6499)-6(n-5)(9 n+25)\right) P_{n-2}^{\prime} \\
& +6 c\left(c^{2}-1\right)(3 n(3 n-44)+659) P_{n-2}^{\prime \prime} \\
& +\left(4 c^{2}(3(n-124) n+553)+3(n-5)(97 n-151)\right) P_{n-4} \\
& -c\left(448 c^{2}(3 n+2)+9 n(11 n-340)+10137\right) P_{n-4}^{\prime} \\
& -3\left(c^{2}-1\right)\left(448 c^{2}+3(n-5)(3 n-37)\right) P_{n-4}^{\prime \prime} \\
& -45(n-7)(n-5) P_{n}-99 c(n-7)(n-5) P_{n}^{\prime} \\
& -27\left(c^{2}-1\right)(n-7)(n-5) P_{n}^{\prime \prime} .
\end{aligned}
$$

De (4.6), $\operatorname{com} n=n-6$ temos que

$$
0=2 c(3 n-7) P_{n-4}-3(n-5) P_{n-6}+(-3 n-1) P_{n-2}
$$

Multiplicando esta equação por $2 c(75-29 n)$ e adicionando-a à equação anterior, obtemos que

$$
\begin{aligned}
0= & \left(112 c^{2}(n(2-3 n)+1)+3(n-5)(97 n-151)\right) P_{n-4} \\
& -c\left(448 c^{2}(3 n+2)+9 n(11 n-340)+10137\right) P_{n-4}^{\prime} \\
& -3\left(c^{2}-1\right)\left(448 c^{2}+3(n-5)(3 n-37)\right) P_{n-4}^{\prime \prime} \\
& +32 c(n(9 n-55)+120) P_{n-2} \\
& +2\left(c^{2}(3 n(51 n-524)+6499)-6(n-5)(9 n+25)\right) P_{n-2}^{\prime} \\
& +6 c\left(c^{2}-1\right)(3 n(3 n-44)+659) P_{n-2}^{\prime \prime} \\
& -45(n-7)(n-5) P_{n}-99 c(n-7)(n-5) P_{n}^{\prime} \\
& -27\left(c^{2}-1\right)(n-7)(n-5) P_{n}^{\prime \prime} .
\end{aligned}
$$

A equação acima não tem índice $n-6$. Agora, queremos eliminar os índices $n-4$. De (4.8) $\operatorname{com} n=n-4$ obtemos que

$$
0=2 c(3 n-1) P_{n-2}^{\prime \prime}+(12 n-4) P_{n-2}^{\prime}+(9-3 n) P_{n-4}^{\prime \prime}+(-3 n-7) P_{n}^{\prime \prime} .
$$

Multiplicando esta equação por $\left(c^{2}-1\right)\left(448 c^{2}+3(n-5)(3 n-37)\right)$ e adicionandoa à $(3-n)$ multiplicado pela equação anterior, obtemos que

$$
\begin{aligned}
0= & +(3-n)\left(112 c^{2}(n(2-3 n)+1)+3(n-5)(97 n-151)\right) P_{n-4} \\
& -c(3-n)\left(448 c^{2}(3 n+2)+9 n(11 n-340)+10137\right) P_{n-4}^{\prime} \\
& +2\left(896 c^{4}(3 n-1)+c^{2}(n(3(359-33 n) n-10261)+19283)+672(n-5)(n-1)\right) P_{n-2}^{\prime} \\
& -32 c(n-3)(n(9 n-55)+120) P_{n-2} \\
& +896 c\left(c^{2}-1\right)\left(c^{2}(3 n-1)-3(n-4)\right) P_{n-2}^{\prime \prime} \\
& +45(n-7)(n-5)(n-3) P_{n}+99 c(n-7)(n-5)(n-3) P_{n}^{\prime} \\
& -448\left(c^{2}-1\right)\left(c^{2}(3 n+7)-3(n-5)\right) P_{n}^{\prime \prime} .
\end{aligned}
$$


De (4.7) $\operatorname{com} n=n-4$ obtemos que

$$
0=2 c(3 n-1) P_{n-2}^{\prime}+(9-3 n) P_{n-4}^{\prime}+(-3 n-7) P_{n}^{\prime}+(6 n-2) P_{n-2} .
$$

Multiplicando esta equação por $c\left(448 c^{2}(3 n+2)+9 n(11 n-340)+10137\right)$ e adicionando-a à equação anterior multiplicada por 3 obtemos que

$$
\begin{aligned}
0= & 3(3-n)\left(112 c^{2}(n(2-3 n)+1)+3(n-5)(97 n-151)\right) P_{n-4} \\
& +2 c\left(448 c^{2}\left(9 n^{2}+3 n-2\right)-3 n(n(45 n+1781)-6597)+7143\right) P_{n-2} \\
& +448\left(2 c^{4}(3 n-1)(3 n+8)+3 c^{2}(n(4-9 n)+71)+9(n-5)(n-1)\right) P_{n-2}^{\prime} \\
& +2688 c\left(c^{2}-1\right)\left(c^{2}(3 n-1)-3(n-4)\right) P_{n-2}^{\prime \prime} \\
& +135(n-7)(n-5)(n-3) P_{n} \\
& -448 c\left(c^{2}(3 n+2)(3 n+7)-9 n(n+3)+228\right) P_{n}^{\prime} \\
& -1344\left(c^{2}-1\right)\left(c^{2}(3 n+7)-3(n-5)\right) P_{n}^{\prime \prime} .
\end{aligned}
$$

De (4.6) $\operatorname{com} n=n-4$ obtemos que

$$
0=2 c(3 n-1) P_{n-2}+(9-3 n) P_{n-4}+(-3 n-7) P_{n} .
$$

Multiplicando esta equação por $-\left(112 c^{2}(n(2-3 n)+1)+3(n-5)(97 n-151)\right)$ e adicionando-a à equação anterior, obtemos que

$$
\begin{aligned}
0= & 224 c(n+1)(3 n+7)\left(c^{2}(3 n-1)-3(n-4)\right) P_{n-2} \\
& +448\left(2 c^{4}(3 n-1)(3 n+8)+3 c^{2}(n(4-9 n)+71)+9(n-5)(n-1)\right) P_{n-2}^{\prime} \\
& +2688 c\left(c^{2}-1\right)\left(c^{2}(3 n-1)-3(n-4)\right) P_{n-2}^{\prime \prime} \\
& -112(n-1)(3 n+1)\left(c^{2}(3 n+7)-3(n-5)\right) P_{n} \\
& -448 c\left(c^{2}(3 n+2)(3 n+7)-9 n(n+3)+228\right) P_{n}^{\prime} \\
& -1344\left(c^{2}-1\right)\left(c^{2}(3 n+7)-3(n-5)\right) P_{n}^{\prime \prime},
\end{aligned}
$$

que pode ser reescrita como

$$
\begin{aligned}
0= & +2 c(n+1)(3 n+7)\left(c^{2}(3 n-1)-3(n-4)\right) P_{n-2} \\
& +4\left(2 c^{4}(3 n-1)(3 n+8)+3 c^{2}(n(4-9 n)+71)+9(n-5)(n-1)\right) P_{n-2}^{\prime} \\
& +24 c\left(c^{2}-1\right)\left(c^{2}(3 n-1)-3(n-4)\right) P_{n-2}^{\prime \prime} \\
& +(1-n)(3 n+1)\left(c^{2}(3 n+7)-3(n-5)\right) P_{n} \\
& -4 c\left(c^{2}(3 n+2)(3 n+7)-9 n(n+3)+228\right) P_{n}^{\prime} \\
& -12\left(c^{2}-1\right)\left(c^{2}(3 n+7)-3(n-5)\right) P_{n}^{\prime \prime} .
\end{aligned}
$$

Agora reduzimos o problema a analisar uma equação diferencial com apenas índices do tipo $n$ e $n-2$. Para reduzir isto a uma equação diferencial linear ordinária com índice apenas $n$, precisaremos de mais equações para cancelar todos os termos que não tenham apenas índice $n$. De (4.8) $\operatorname{com} n=n-2$ obtemos que

$$
0=2 c(3 n+5) P_{n}^{\prime \prime}+4(3 n+5) P_{n}^{\prime}+(3-3 n) P_{n-2}^{\prime \prime}+(-3 n-13) P_{n+2}^{\prime \prime} .
$$


Multiplicando esta última equação por $-8 c\left(c^{2}-1\right)\left(c^{2}(3 n-1)-3(n-4)\right)$ e adicionandoa à equação anterior multiplicada por $(1-n)$ obtemos que

$$
\begin{aligned}
0= & -2 c(n+1)(3 n+7)(n-1)\left(c^{2}(3 n-1)-3(n-4)\right) P_{n-2} \\
& +4(1-n)\left(2 c^{4}(3 n-1)(3 n+8)+3 c^{2}(n(4-9 n)+71)+9(n-5)(n-1)\right) P_{n-2}^{\prime} \\
& +8 c\left(c^{2}-1\right)(3 n+13)\left(c^{2}(3 n-1)-3(n-4)\right) P_{n+2}^{\prime \prime} \\
& +(3 n+1)(n-1)^{2}\left(c^{2}(3 n+7)-3(n-5)\right) P_{n} \\
& +4 c\left(-8 c^{4}(3 n-1)(3 n+5)+c^{2}(n(9 n(n+18)-85)-534)-9 n(n(n+10)-47)+252\right) P_{n}^{\prime} \\
& +4\left(c^{2}-1\right)\left(-4 c^{4}(3 n-1)(3 n+5)+9 c^{2}(n(5 n-8)-29)-9(n-5)(n-1)\right) P_{n}^{\prime \prime} .
\end{aligned}
$$

De (4.7) $\operatorname{com} n=n-2$ obtemos que

$$
0=2 c(3 n+5) P_{n}^{\prime}+(3-3 n) P_{n-2}^{\prime}+(-3 n-13) P_{n+2}^{\prime}+(6 n+10) P_{n} .
$$

Multiplicando esta equação por

$$
4\left(9(-5+n)(-1+n)+2 c^{4}(-1+3 n)(8+3 n)+3 c^{2}(71+(4-9 n) n)\right)
$$

e adicionando-a à equação anterior multiplicada por $(-3)$ obtemos que

$$
\begin{aligned}
0= & +6 c(n-1)(n+1)(3 n+7)\left(c^{2}(3 n-1)-3(n-4)\right) P_{n-2} \\
& +4(-3 n-13)\left(2 c^{4}(3 n-1)(3 n+8)+3 c^{2}(n(4-9 n)+71)+9(n-5)(n-1)\right) P_{n+2}^{\prime} \\
& -24 c\left(c^{2}-1\right)(3 n+13)\left(c^{2}(3 n-1)-3(n-4)\right) P_{n+2}^{\prime \prime} \\
& +\left(16 c^{4}(3 n-1)(3 n+5)(3 n+8)-3 c^{2}(n(n(3 n(3 n+74)+232)-1854)-2833)\right. \\
& +9(n-5)(n-1)(n+3)(3 n+13)) P_{n} \\
& +4\left(4 c^{5}(3 n-1)(3 n+5)(3 n+14)+3 c^{3}(n(551-3 n(21 n+76))+1244)\right. \\
& +9 c(n(n(9 n+4)-171)-34)) P_{n}^{\prime} \\
& -12\left(c^{2}-1\right)\left(-4 c^{4}(3 n-1)(3 n+5)+9 c^{2}(n(5 n-8)-29)-9(n-5)(n-1)\right) P_{n}^{\prime \prime} .
\end{aligned}
$$

Agora vamos eliminar os termos do tipo $P_{n-2}$ multiplicando (4.6) $\operatorname{com} n=n-2$

$$
0=2 c(3 n+5) P_{n}+(3-3 n) P_{n-2}+(-3 n-13) P_{n+2}
$$

por

$$
2 c(n+1)(3 n+7)\left(c^{2}(3 n-1)-3(n-4)\right)
$$

e adicionando-a à equação anterior para obter

$$
\begin{aligned}
0= & -2 c(n+1)(3 n+7)(3 n+13)\left(c^{2}(3 n-1)-3(n-4)\right) P_{n+2} \\
& +4(-3 n-13)\left(2 c^{4}(3 n-1)(3 n+8)+3 c^{2}(n(4-9 n)+71)+9(n-5)(n-1)\right) P_{n+2}^{\prime} \\
& -24 c\left(c^{2}-1\right)(3 n+13)\left(c^{2}(3 n-1)-3(n-4)\right) P_{n+2}^{\prime \prime} \\
& +(n+3)(3 n+13)\left(4 c^{4}(3 n-1)(3 n+5)+9 c^{2}(n(8-5 n)+29)+9(n-5)(n-1)\right) P_{n} \\
& +4\left(4 c^{5}(3 n-1)(3 n+5)(3 n+14)+3 c^{3}(n(551-3 n(21 n+76))+1244)\right. \\
& +9 c(n(n(9 n+4)-171)-34)) P_{n}^{\prime} \\
& -12\left(c^{2}-1\right)\left(-4 c^{4}(3 n-1)(3 n+5)+9 c^{2}(n(5 n-8)-29)-9(n-5)(n-1)\right) P_{n}^{\prime \prime} .
\end{aligned}
$$


Fazendo $n=n-2$ na equação acima, obtemos que

$$
\begin{aligned}
0= & (n+1)(3 n+7)\left(4 c^{4}(3 n(3 n-8)+7)-9 c^{2}(n(5 n-28)+7)+9(n-7)(n-3)\right) P_{n-2} \\
& +4\left(4 c^{5}\left(27 n^{3}-171 n+56\right)+3 c^{3}(n(3 n(50-21 n)+707)-266)\right. \\
& +9 c(n(n(9 n-50)-79)+252)) P_{n-2}^{\prime} \\
& -12\left(c^{2}-1\right)\left(-4 c^{4}(3 n(3 n-8)+7)+9 c^{2}(n(5 n-28)+7)\right. \\
& -9(n-7)(n-3)) P_{n-2}^{\prime \prime} \\
& -2 c(n-1)(3 n+1)(3 n+7)\left(c^{2}(3 n-7)-3(n-6)\right) P_{n} \\
& +4(-3 n-7)\left(2 c^{4}(3 n-7)(3 n+2)+3 c^{2}(n(40-9 n)+27)+9(n-7)(n-3)\right) P_{n}^{\prime} \\
& -24 c\left(c^{2}-1\right)(3 n+7)\left(c^{2}(3 n-7)-3(n-6)\right) P_{n}^{\prime \prime} .
\end{aligned}
$$

Podemos comparar esta equação à equação (4.9)

$$
\begin{aligned}
0= & +2 c(n+1)(3 n+7)\left(c^{2}(3 n-1)-3(n-4)\right) P_{n-2} \\
& +4\left(2 c^{4}(3 n-1)(3 n+8)+3 c^{2}(n(4-9 n)+71)+9(n-5)(n-1)\right) P_{n-2}^{\prime} \\
& +24 c\left(c^{2}-1\right)\left(c^{2}(3 n-1)-3(n-4)\right) P_{n-2}^{\prime \prime} \\
& +(1-n)(3 n+1)\left(c^{2}(3 n+7)-3(n-5)\right) P_{n} \\
& -4 c\left(c^{2}(3 n+2)(3 n+7)-9 n(n+3)+228\right) P_{n}^{\prime} \\
& -12\left(c^{2}-1\right)\left(c^{2}(3 n+7)-3(n-5)\right) P_{n}^{\prime \prime} .
\end{aligned}
$$

O nosso objetivo é eliminar os termos do tipo $P_{n-2}^{\prime \prime}$, os termos do tipo $P_{n-2}^{\prime}$ e finalmente os termos $P_{n-2}$ para chegar em uma equação diferencial com apenas $P_{n}$ e suas derivadas. Primeiro iremos diminuir o grau de $c$ no polinômio em frente de $P_{n-2}^{\prime \prime}$. Multiplicaremos esta equação por $c(14-6 n)$ e adicionaremos ela à equação anterior para obter

$$
\begin{aligned}
0= & -3(n-7)(n+1)(3 n+7)\left(c^{2}(3 n+13)-3 n+9\right) P_{n-2} \\
& -12 c\left(c^{2}(n-7)(3 n+8)(3 n+13)-9 n^{3}+579 n-966\right) P_{n-2}^{\prime} \\
& -36\left(c^{2}-1\right)(n-7)\left(c^{2}(3 n+13)-3 n+9\right) P_{n-2}^{\prime \prime} \\
& +66 c(n-7)(n-1)(3 n+1) P_{n} \\
& +12(n-7)\left(c^{2}\left(9 n^{2}-6 n+179\right)-9 n^{2}+6 n+63\right) P_{n}^{\prime} \\
& +792 c\left(c^{2}-1\right)(n-7) P_{n}^{\prime \prime} .
\end{aligned}
$$

Caso $n \neq 7$, teremos que

$$
\begin{aligned}
0= & -(n+1)(3 n+7)\left(c^{2}(3 n+13)-3 n+9\right) P_{n-2} \\
& -4 c\left(c^{2}(3 n+8)(3 n+13)-9 n(n+7)+138\right) P_{n-2}^{\prime} \\
& -12\left(c^{2}-1\right)\left(c^{2}(3 n+13)-3 n+9\right) P_{n-2}^{\prime \prime} \\
& +264 c\left(c^{2}-1\right) P_{n}^{\prime \prime} \\
& +4\left(c^{2}\left(9 n^{2}-6 n+179\right)-9 n^{2}+6 n+63\right) P_{n}^{\prime} \\
& +22 c(n-1)(3 n+1) P_{n} .
\end{aligned}
$$

Diminuiremos o grau do polinômio em $c$ em frente de $P_{n-2}^{\prime \prime}$ mais uma vez multiplicando a equação anterior por $(2 c(-1+3 n))$ e adicionando o resultado à (4.10) 
multiplicada por $(13+3 n)$ para obter

$$
\begin{aligned}
0= & -66 c(n-5)(n+1)(3 n+7) P_{n-2} \\
& -12(n-5)\left(c^{2}\left(9 n^{2}+30 n+203\right)-3(n-1)(3 n+13)\right) P_{n-2}^{\prime} \\
& -792 c\left(c^{2}-1\right)(n-5) P_{n-2}^{\prime \prime} \\
& -3(n-5)(n-1)(3 n+1)\left(3 c^{2}(n-3)-3 n-13\right) P_{n} \\
& +12 c(n-5)\left(3 c^{2}(n-3)(3 n-4)-9 n^{2}+39 n+206\right) P_{n}^{\prime} \\
& -36\left(c^{2}-1\right)(n-5)\left(3 c^{2}(n-3)-3 n-13\right) P_{n}^{\prime \prime} .
\end{aligned}
$$

Portanto, se $n \neq 5$ e $n \neq 7$, teremos que

$$
\begin{aligned}
0= & -66 c(n+1)(3 n+7) P_{n-2} \\
& -12\left(c^{2}\left(9 n^{2}+30 n+203\right)-3(n-1)(3 n+13)\right) P_{n-2}^{\prime} \\
& -792 c\left(c^{2}-1\right) P_{n-2}^{\prime \prime} \\
& +12 c\left(3 c^{2}(n-3)(3 n-4)-9 n^{2}+39 n+206\right) P_{n}^{\prime} \\
& -36\left(c^{2}-1\right)\left(3 c^{2}(n-3)-3 n-13\right) P_{n}^{\prime \prime} \\
& -3(n-1)(3 n+1)\left(3 c^{2}(n-3)-3 n-13\right) P_{n} .
\end{aligned}
$$

Podemos reescrever a equação acima como

$$
\begin{aligned}
0= & -22 c(n+1)(3 n+7) P_{n-2} \\
& +\left(12(n-1)(3 n+13)-4 c^{2}\left(9 n^{2}+30 n+203\right)\right) P_{n-2}^{\prime} \\
& -264 c\left(c^{2}-1\right) P_{n-2}^{\prime \prime} \\
& -(n-1)(3 n+1)\left(3 c^{2}(n-3)-3 n-13\right) P_{n} \\
& +4 c\left(3 c^{2}(n-3)(3 n-4)-9 n^{2}+39 n+206\right) P_{n}^{\prime} \\
& -12\left(c^{2}-1\right)\left(3 c^{2}(n-3)-3 n-13\right) P_{n}^{\prime \prime} .
\end{aligned}
$$

Abaixamos o grau de $c$ no polinômio em frente de $P_{n-2}^{\prime \prime}$ mais uma vez multiplicando esta última equação por $(c(13+3 n))$ e adicionando à equação (4.11) multiplicada por $(-22)$ para obter

$$
\begin{aligned}
0= & -66(n-3)(n+1)(3 n+7) P_{n-2} \\
& -12 c(n-3)\left(3 c^{2}(n-1)(3 n+13)-3 n(3 n+10)+281\right) P_{n-2}^{\prime} \\
& -792\left(c^{2}-1\right)(n-3) P_{n-2}^{\prime \prime} \\
& +12(n-3)\left(c^{2}(3 n-4)\left(c^{2}(3 n+13)-3 n-35\right)+22(3 n+7)\right) P_{n}^{\prime} \\
& -36 c\left(c^{2}-1\right)(n-3)\left(c^{2}(3 n+13)-3 n-35\right) P_{n}^{\prime \prime} \\
& -3 c(n-3)(n-1)(3 n+1)\left(c^{2}(3 n+13)-3 n-35\right) P_{n} .
\end{aligned}
$$

Portanto, se $n \neq 5, n \neq 7$ e $n \neq 3$ teremos que

$$
\begin{aligned}
0= & -22(n+1)(3 n+7) P_{n-2} \\
& -4 c\left(3 c^{2}(n-1)(3 n+13)-3 n(3 n+10)+281\right) P_{n-2}^{\prime} \\
& -264\left(c^{2}-1\right) P_{n-2}^{\prime \prime} \\
& -c(n-1)(3 n+1)\left(c^{2}(3 n+13)-3 n-35\right) P_{n} \\
& +\left(4 c^{2}(3 n-4)\left(c^{2}(3 n+13)-3 n-35\right)+88(3 n+7)\right) P_{n}^{\prime} \\
& -12 c\left(c^{2}-1\right)\left(c^{2}(3 n+13)-3 n-35\right) P_{n}^{\prime \prime} .
\end{aligned}
$$


Agora queremos eliminar os termos com $P_{n-2}^{\prime \prime}$. Isso será feito multiplicando a equação anterior por $(-3 c)$ e adicionando o resultado à equação (4.12) para obter

$$
\begin{aligned}
0= & 3\left(c^{2}-1\right)^{2}(n-1)(3 n+1)(3 n+13) P_{n} \\
& -12 c\left(c^{2}-1\right)^{2}(3 n-4)(3 n+13) P_{n}^{\prime} \\
& +36\left(c^{2}-1\right)^{3}(3 n+13) P_{n}^{\prime \prime} \\
& +36\left(c^{2}-1\right)^{2}(n-1)(3 n+13) P_{n-2}^{\prime} .
\end{aligned}
$$

Como $c \in \mathbb{C} \backslash\{-1,1\}$ e $n \neq 5, n \neq 7, n \neq 3$ e $n \neq-13 / 3$ teremos que

$$
0=12\left(c^{2}-1\right) P_{n}^{\prime \prime}+4 c(4-3 n) P_{n}^{\prime}+12(n-1) P_{n-2}^{\prime}+(n-1)(3 n+1) P_{n} .
$$

Se diferenciarmos esta equação em relação à c, obteremos que

$$
0=12\left(c^{2}-1\right) P_{n}^{(3)}+4 c(10-3 n) P_{n}^{\prime \prime}+(n-3)(3 n-5) P_{n}^{\prime}+12(n-1) P_{n-2}^{\prime \prime} .
$$

Agora, eliminaremos o termo do tipo $P_{n-2}^{\prime}$. Multiplicaremos a equação anterior por $22\left(1-c^{2}\right)$ e a adicionaremos à (4.13) multiplicada por $(-1+n)$ para obter

$$
\begin{aligned}
0= & -22(n-1)(n+1)(3 n+7) P_{n-2} \\
& -4 c(n-1)\left(3 c^{2}(n-1)(3 n+13)-3 n(3 n+10)+281\right) P_{n-2}^{\prime} \\
& -c(n-1)^{2}(3 n+1)\left(c^{2}(3 n+13)-3 n-35\right) P_{n} \\
& +\left(22\left(c^{2}-1\right)(n-3)(3 n-5)+(n-1)\right. \\
& \left.\left(4 c^{2}(3 n-4)\left(c^{2}(3 n+13)-3 n-35\right)+88(3 n+7)\right)\right) P_{n}^{\prime} \\
& +4 c\left(c^{2}-1\right)\left(-3 c^{2}(n-1)(3 n+13)+9 n^{2}+30 n+115\right) P_{n}^{\prime \prime} \\
& +264\left(c^{2}-1\right)^{2} P_{n}{ }^{(3)} .
\end{aligned}
$$

Multiplicando a equação (4.14) por

$$
\left(-c\left(9 c^{2} n^{2}+30 c^{2} n-39 c^{2}-9 n^{2}-30 n+281\right)\right)
$$

e adicionando-a à (-3) multiplicado à equação anterior, obtemos que

$$
\begin{aligned}
0= & +6(n-1)(n+1)(3 n+7) P_{n-2} \\
& -2 c(n-1)(3 n+1)(3 n+8) P_{n} \\
& \left(2 c^{2}(9 n(3 n+10)-173)-6\left(9 n^{2}+30 n-43\right)\right) P_{n}^{\prime} \\
& -432 c\left(c^{2}-1\right) P_{n}^{\prime \prime} \\
& -72\left(c^{2}-1\right)^{2} P_{n}{ }^{(3)} .
\end{aligned}
$$

Se diferenciarmos a equação acima em relação à $c$ obtemos que

$$
\begin{aligned}
0= & 3(n-1)(n+1)(3 n+7) P_{n-2}^{\prime}-(n-1)(3 n+1)(3 n+8) P_{n} \\
& +c(n(199-9(n-4) n)-338) P_{n}^{\prime} \\
& +\left(c^{2}(9 n(3 n+10)-821)-9 n(3 n+10)+345\right) P_{n}^{\prime \prime} \\
& -36\left(c^{2}-1\right)^{2} P_{n}{ }^{(4)}-360 c\left(c^{2}-1\right) P_{n}{ }^{(3)} .
\end{aligned}
$$

Agora, multiplicando esta equação por $(-4)$ e adicionando-a à equação (4.14) multiplicada por $(n+1)(3 n+7)$ obtemos uma equação diferencial ordinária linear de ordem 4 satisfeita pelos polinômios $P_{n}$. Provamos o seguinte teorema. 
Teorema 4.4.1 Os polinômios $P_{n}=P_{-1, n}$ satisfazem a seguinte equação diferencial linear ordinária de ordem 4.

$$
\begin{aligned}
& 144\left(c^{2}-1\right)^{2} P_{n}^{(4)}+1440 c\left(c^{2}-1\right) P_{n}^{(3)} \\
& -8\left(c^{2}\left(9 n^{2}+30 n-421\right)-3 n(3 n+10)+183\right) P_{n}^{\prime \prime} \\
& -24 c\left(9 n^{2}+30 n-61\right) P_{n}^{\prime}+(n-1)(n+3)(3 n+1)(3 n+13) P_{n}=0 .
\end{aligned}
$$

\section{Caso 2}

Reindexaremos os polinômios $P_{-3, n}$ da seguinte forma:

$$
P_{-3}(c, z):=z^{5 / 3} \sqrt[3]{-2 c z^{2}+z^{4}+1} \int \frac{4 z^{1 / 3}}{3\left(-2 c z^{2}+z^{4}+1\right)^{4 / 3}} \mathrm{~d} z=\sum_{n=0}^{\infty} P_{-3, n}(c) z^{n} .
$$

Isto significa que $P_{-3,1}=1$, e $P_{-3,0}=P_{-3,2}=P_{-3,3}=P_{-3,4}=0$ e $P_{-3, n}$ satisfaz a seguinte relação de recursividade

$$
(7+3 n) P_{n}(c)=-3(n-3) P_{n-4}(c)+2 c(2+3(-1+n)) P_{n-2}(c) .
$$

Os primeiros polinômios não nulos em $c$ são

$$
\begin{aligned}
P_{-3,5}(c) & =\frac{3}{5}, & P_{-3,7}(c)=\frac{3 c}{5} \\
P_{-3,9}(c) & =\frac{3}{55}\left(14 c^{2}-3\right), & P_{-3,11}(c)=\frac{3}{55} c\left(20 c^{2}-9\right), \\
\text { e } P_{-3,13}(c) & =3 / 935\left(27-360 c^{2}+520 c^{4}\right) &
\end{aligned}
$$

e então,

$$
\begin{aligned}
P_{-3}(c, z)= & z+\frac{3 z^{5}}{5}+\frac{3 c z^{7}}{5}+\frac{3}{55}\left(14 c^{2}-3\right) z^{9}+\frac{1}{55}(3 c)\left(20 c^{2}-9\right) z^{11} \\
& +\frac{3}{935}\left(520 c^{4}-360 c^{2}+27\right) z^{13}+O\left(z^{14}\right) .
\end{aligned}
$$

Depois de uma análise muito similar e tão longa quanto a do Caso 1 chegamos no seguinte resultado que expressa a mesma equação diferencial de ordem 4 .

Teorema 4.4.2 Os polinômios $P_{n}=P_{-3, n}$ satisfazem a seguinte equação diferencial linear ordinária de ordem 4.

$$
\begin{aligned}
& 144\left(c^{2}-1\right)^{2} P_{n}{ }^{(4)}+1440 c\left(c^{2}-1\right) P_{n}{ }^{(3)} \\
& -8\left(c^{2}\left(9 n^{2}+30 n-421\right)-3 n(3 n+10)+183\right) P_{n}^{\prime \prime} \\
& -24 c\left(9 n^{2}+30 n-61\right) P_{n}^{\prime}+(n-1)(n+3)(3 n+1)(3 n+13) P_{n}=0 .
\end{aligned}
$$

\subsubsection{Polinômios ultraesféricos associados}

Os polinômios $P_{-1, n}$ e $P_{-3, n}$ satisfazem a seguinte relação de recursividade

$$
(3 n-5) P_{n-4}=2 c(3 n-13) P_{n-6}+3(7-n) P_{n-8}
$$

Note que, em ambos os casos os polinômios com índices pares são nulos. Agora, vamos reescrever a equação aicma $\operatorname{com} n=2 n+1$ e considerando $q_{s}(c):=P_{2 s+5}$. Então teremos que

$$
(6 n-2) q_{n-4}=4 c(3 n-5) q_{n-5}+3(6-2 n) q_{n-6}
$$


em que $q_{s}=q_{s}(c)$. Reescrevendo novamente considerando $n=n+5$, obtemos que

$$
2 c(3 n+10) q_{n}=(3 n+6) q_{n-1}+(3 n+14) q_{n+1}
$$

Para o caso $q_{s}=P_{-3,2 s+5}$ temos que

$$
\begin{array}{cc}
q_{-2}=P_{-3,1}=1, & q_{0}=P_{-3,5}=\frac{3}{5} \\
q_{1}=P_{-3,7}=\frac{3 c}{5}, & q_{2}=P_{-3,9}=\frac{3}{55}\left(14 c^{2}-3\right) \\
q_{3}=P_{-3,11}=\frac{3}{55} c\left(20 c^{2}-9\right), & q_{4}=P_{-3,13}=\frac{3}{935}\left(520 c^{4}-360 c^{2}+27\right) .
\end{array}
$$

Mostraremos que esta família é um caso especial de polinômios ultraesféricos associados. Para $c$ e $v$ duas constantes complexas, seja $C_{n}^{(v)}(\chi ; c)$ a família de polinômios ultraesféricos associados com condições iniciais $C_{-1}^{(v)}(\chi ; c)=0$ e $C_{0}^{(v)}(\chi ; c)=1$ definidos como a família que satisfaz a seguinte relação

$$
2 \chi(n+v+c) C_{n}^{(v)}(\chi ; c)=(n+c+1) C_{n+1}^{(v)}(\chi ; c)+(2 v+n+c-1) C_{n-1}^{(v)}(\chi ; c) .
$$

Então definindo na equação acima $c=11 / 3$ e $v=-1 / 3$ obtemos a mesma relação de recursividade descrita em (4.15). Portanto, podemos concluir que os polinômios do tipo $P_{-3, n}$ são um caso especial de polinômios ultraesféricos associados.

Considere $\alpha, \beta, c \in \mathbb{C}$ constantes e os polinômios de Jacobi associados $P_{n}^{(\alpha, \beta)}(\chi ; c)$ definidos como a família que satisfaz a seguinte relação de recursividade

$$
\begin{aligned}
& 2(n+c+1)(n+c+\gamma)(2 n+2 c+\gamma-1) p_{n+1} \\
\quad & =(2 n+2 c+\gamma)((2 n+2 c+\gamma-1)(2 n+2 c+\gamma+1) \chi \\
+ & (\gamma-1)(\gamma-2 \beta-1)) p_{n} \\
- & 2(n+c+\gamma-\beta-1)(n+c+\beta)(2 n+2 c+\gamma+1) p_{n-1},
\end{aligned}
$$

em que $n \in \mathbb{N}, \gamma=\alpha+\beta+1, P_{-1}^{(\alpha, \beta)}(\chi ; c)=0 ; P_{0}^{(\alpha, \beta)}(\chi ; c)=1$. Seguindo [CFT13], é possível verificar que os polinômios ultraesféricos associados se relacionam com os polinômios de Jacobi ultraesféricos por intermédio da seguinte relação

$$
P_{n}^{(v-1 / 2, v-1 / 2)}(\chi ; \beta)=\frac{\left(v+c+\frac{1}{2}\right) n}{(2 v+c)_{n}} C_{n}^{(v)}(\chi ; \beta) .
$$

A função peso para os polinômios ultraesféricos associados também é conhecida e é dada por

$$
\rho(\cos t)=\frac{(2 \sin t)^{2 \beta-1}(\Gamma(\lambda+\gamma))^{2}}{2 \pi \Gamma(2 \beta+\gamma) \Gamma(\gamma+1)}\left|{ }_{2} F_{1}\left(1-\beta, \gamma ; \gamma+\beta ; e^{2 i t}\right)\right|^{2}
$$

para $0 \leq t \leq \pi$ e em que ${ }_{2} F_{1}(a, b ; c ; z)$ é a função hipergeométrica

$$
{ }_{2} F_{1}(a, b ; c, z)=\sum_{n=0}^{\infty} \frac{(a)_{n}(b)_{n}}{(c)_{n}} z^{n}
$$

Teorema 4.4.3 Os polinômios $P_{-3, n}$ são polinômios ortogonais não-classicos. 
Demonstração: Resgatando o que já foi apresentado na Seção 4.2, os polinômios de Jacobi associados satisfazem a seguinte equação diferencial de ordem quatro

$$
A_{0}(x) y^{(i v)}+A_{1}(x) y^{\prime \prime \prime}+A_{2}(x) y^{\prime \prime}+A_{3}(x) y^{\prime}+A_{4}(x) y=0,
$$

em que

$$
\begin{aligned}
& A_{0}(x)=\left(1-x^{2}\right)^{2}, \\
& A_{1}(x)=10 x\left(x^{2}-1\right), \\
& A_{2}(x)=-(1-x)^{2}\left(2 K+2 C+\gamma^{2}-25\right)+2(1-x)(2 K+2 C+2 \alpha \gamma)+2(\alpha+1)-26, \\
& A_{3}(x)=3(1-x)\left(2 K+2 C+\gamma^{2}-5\right)-6(K+C+\alpha \gamma+\beta-2), \\
& A_{4}(x)=n(n+2)(n+\gamma+2 c)(n+\gamma+2 c-2),
\end{aligned}
$$

em que

$$
K=(n+c)(n+\gamma+c), \quad C=(c-1)(c+\alpha+\beta) .
$$

No caso dos polinômios $q_{n}(x)=P_{-3,2 n+5}(x)=C_{n}^{-1 / 3}(x, 11 / 3)$ para $n \geq-1$, a equação diferencial de ordem 4 que chegamos tem a forma

$$
\begin{aligned}
& \left(96(n(3 n+20)+16)-16 x^{2}(6 n(3 n+20)-23)\right) q_{n}^{\prime \prime} \\
& +144\left(x^{2}-1\right)^{2} q_{n}{ }^{(4)}+1440 x\left(x^{2}-1\right) q_{n}{ }^{(3)}-48 x(6 n(3 n+20)+157) q_{n}^{\prime} \\
& +16(n+2)(n+4)(3 n+8)(3 n+14) q_{n}=0 .
\end{aligned}
$$

Para $n=2$ temos que $q_{2}(x)=\frac{3}{55}\left(14 x^{2}-3\right)$, juntando esta informação à equação acima, obtemos que

$$
16 x^{2}-3=0 .
$$

Teremos que $c=11 / 3, v=-1 / 3, \alpha=\beta=-5 / 6$ e $\gamma=-2 / 3$

$$
\begin{aligned}
& A_{2}(x)=\frac{1}{9}\left(-6 n(3 n+20)\left(x^{2}-1\right)-x(73 x+462)+304\right) \\
& A_{3}(x)=-\frac{1}{3}(6 n(3 n+20)+253) x \\
& A_{4}(s)=n(n+2)\left(n+\frac{14}{3}\right)\left(n+\frac{20}{3}\right)
\end{aligned}
$$

Quando $n=2$ vemos que $A_{2}(x)$ é diferente do coeficiente correspondente e $q_{2}(x)=$ $\frac{3}{55}\left(14 x^{2}-3\right)$ não satisfaz a equação acima. Então as famílias de equações diferenciais de ordem 4 encontradas neste trabalho são diferentes daquelas apresentadas por Ismail em [Ism05] para polinômios ultraesféricos associados, confirmando o que foi observado em [CFT13].

Procedendo de forma similar podemos demonstrar o seguinte resultado.

Teorema 4.4.4 Os polinômios $P_{-1, n}$ são polinômios ortogonais não-clássicos.

\subsubsection{Ortogonalidade de $P_{-1, n}$ e $P_{-3, n}$}

Os polinômios do $P_{-1, n}$ e $P_{-3, n}$ satisfazem a mesma relação de recursividade e equação diferencial de ordem 4 , mas tem condições iniciais diferentes. Sendo assim, sabemos imediatamente que são polinômios ultraesféricos associados. Aqui provaremos de forma independente que estes polinômios são ortogonais. Considerando que 
$q_{s}(c)=P_{-1,2 s+1}$, desejamos polinômios com índice $n$ igual ao grau do polinômio, então iremos definir que

$$
\bar{q}_{n}:=q_{n-1}, \quad n \geq 0 .
$$

e ignorando os "primeiros"dois polinômios $q_{-1}$ e $q_{0}$, teremos que

$$
\bar{q}_{0}=1, \quad \bar{q}_{1}=\frac{2 c}{5}, \quad \bar{q}_{2}=\frac{2 c^{2}}{5}, \quad \bar{q}_{3}=\frac{2}{55} c\left(14 c^{2}-3\right)
$$

e tendo a relação de comutatividade para $P_{n}$ dada por

$$
(3 n+7) P_{n}=2 c(3 n-1) P_{n-2}+3(3-n) P_{n-4},
$$

então

$$
(3 n+7) P_{n}=2 c(3 n-1) P_{n-2}+3(3-n) P_{n-4}
$$

ou

$$
2 c(3 n+4) \bar{q}_{n+1}=3 n \bar{q}_{n}+(3 n+8) \bar{q}_{n+2,}
$$

em que os polinômios $\bar{q}_{n}$ são de grau $n$ em $c$. Considere o seguinte resultado.

Teorema 4.4.5 ([CFT13] , Teorema 5.0.4) Seja $\left\{p_{n}, n \geq 0\right\}$ uma sequência de polinômios em que $p_{n}$ é um polinômio de grau $n$ satisfazendo a seguinte relação de recursividade

$$
\chi p_{n}=a_{n+1} p_{n+1}+b_{n} p_{n}+c_{n-1} p_{n-1}, n=0,1,2, \ldots,
$$

para números complexos $a_{n+1}, b_{n}, c_{n-1}, n=0,1,2, \ldots$ e $p_{-1}=0$. Então $\left\{p_{n}, n \geq 0\right\}$ é uma sequência de polinômios ortonormais com respeito a uma (única) função de peso se e somente se $b_{n} \in \mathbb{R}$ e $c_{n}=\bar{a}_{n+1} \neq 0$ para todo $n \geq 0$.

Teorema 4.4.6 Os polinômios $P_{-1, n}(c)$ são ortogonais com respeito a alguma função de peso.

Demonstração: Para mostrar que estes polinômios são ortogonais é suficiente verificar a existência de uma família de polinômios ortogonais $f_{n}$ e constantes $\lambda_{n}$ tais que $q_{n}=\lambda_{n} f_{n}$ para todo $n$. Temos a seguinte relação de recursividade para $f_{n}$ :

$$
2 c(3 n+4) f_{n+1} \lambda_{n+1}=3 n f_{n} \lambda_{n}+(3 n+8) f_{n+2} \lambda_{n+2}
$$

ou

$$
c f_{n+1}=\frac{(3 n+8) f_{n+2} \lambda_{n+2}}{2(3 n+4) \lambda_{n+1}}+\frac{(3 n) f_{n} \lambda_{n}}{2(3 n+4) \lambda_{n+1}}
$$

para $n \geq 0$. Considere

$$
A_{n}=\frac{(3 n) f_{n} \lambda_{n}}{2(3 n+4) \lambda_{n+1}}, \quad C_{n+2}=\frac{(3 n+8) f_{n+2} \lambda_{n+2}}{2(3 n+4) \lambda_{n+1}} .
$$

Então $A_{n}=C_{n-1}$ se e somente se

$$
\frac{(3(n-1)+8) f_{n+1} \lambda_{n+1}}{2(3(n-1)+4) \lambda_{n}}=\frac{(3 n) f_{n} \lambda_{n}}{2(3 n+4) \lambda_{n+1}}
$$

ou

$$
\lambda_{n}^{2}=\frac{\left((3 n+4)(3 n+5) f_{n+1}\right) \lambda_{n+1}^{2}}{3 n(3 n+1) f_{n}} .
$$

Tomando $\lambda_{0}=1$ podemos encontrar uma família de constantes $\lambda_{n}$ que satisfazem esta relação. Portanto, pelo Teorema 4.4.5, os polinômios $f_{n}$ formam uma família de polinômios ortogonais em relação à alguma medida e portanto, os polinômios são ortogonais. 
De forma similar podemos demonstrar o seguinte resultado.

Teorema 4.4.7 Os polinômios $P_{-3, n}(c)$ são ortogonais com respeito a alguma função de peso.

O leitor pode desejar comparar as equações diferenciais obtidas em [CFT13] e neste trabalho.

\section{Equações obtidas do caso DJKM}

Em [CFT13] é obtida uma família de polinômios ortogonais e não-clássicos que satisfaz a seguinte equação diferencial de ordem quatro:

$$
\begin{aligned}
& 16\left(c^{2}-1\right)^{2} P_{n}^{(i v)}+160 c\left(c^{2}-1\right) P_{n}^{\prime \prime \prime}-8\left(c^{2}\left(n^{2}-4 n-42\right)-n^{2}+4 n+18\right) P_{n}^{\prime \prime} \\
& -24 c\left(n^{2}-4 n-2\right) P_{n}^{\prime}+(n-6)(n-2)^{2}(n+2) P_{n}=0 .
\end{aligned}
$$

Além disso, no mesmo trabalho é obtida uma família de polinômios ortogonais e não-clássicos que satisfaz a seguinte equação diferencial de ordem quatro:

$$
\begin{aligned}
& 16\left(c^{2}-1\right)^{2} P_{n}^{(i v)}+160 c\left(c^{2}-1\right) P_{n}^{\prime \prime \prime}-8\left(c^{2}\left(n^{2}-4 n-46\right)-n^{2}+4 n+22\right) P_{n}^{\prime \prime} \\
& -24 c\left(n^{2}-4 n-6\right) P_{n}^{\prime}+(n-4)^{2} n^{2} P_{n}=0 .
\end{aligned}
$$

\section{Equação obtida neste trabalho}

Neste trabalho são obtidas duas famílias de polinômios ortogonais e não-clássicos que satisfazem a seguinte equação diferencial de ordem quatro

$$
\begin{aligned}
& 144\left(c^{2}-1\right)^{2} P_{n}^{(4)}+1440 c\left(c^{2}-1\right) P_{n}{ }^{(3)} \\
& -8\left(c^{2}\left(9 n^{2}+30 n-421\right)-3 n(3 n+10)+183\right) P_{n}^{\prime \prime} \\
& -24 c\left(9 n^{2}+30 n-61\right) P_{n}^{\prime}+(n-1)(n+3)(3 n+1)(3 n+13) P_{n}=0 .
\end{aligned}
$$




\section{Capítulo 5}

\section{Representações de álgebras de Krichever-Novikov}

As álgebras de Lie afim são o tipo de álgebras de dimensão infinita de Kac-Moody mais estudadas. Uma das razões pelo interesse por estas álgebras é a rica teoria de representações que pode ser desenvolvida de forma ainda mais complexa do que a de álgebras de Lie simples de dimensão finita. Em particular, as álgebras de Lie afim possuem módulos irredutíveis que contém espaços de peso de dimensão finita e infinita, algo que não pode ocorrer no contexto de álgebras de dimensão finita. É possível decompor as álgebras de Lie afim em partições que não são padrão (veja [Fut97]). A cada partição é possível corresponder uma subálgebra de Borel e é possível formar representações induzidas por módulos de dimensão 1 dessas subálgebras de Borel. Estes módulos obtidos são chamados de módulos do tipo Verma e foram estudados a princípio por Jakobsen e Kac (veja [JK89]) e por Futorny (veja [Fut96] e [Fut97]).

A classificação de módulos irredutíveis é conhecida apenas para módulos com espaços de peso de dimensão finita (veja [FT01]) e para algumas subcategorias de módulos induzidos com apenas alguns espaços de peso de dimensão infinita. Neste capítulo, seguindo o trabalho de Bekkert, Benkart, Futorny e Kashuba (veja [Bek+13]), definimos a álgebra hiperelíptica de Heisenberg como a subálgebra de uma álgebra de Krichever-Novikov hiperelíptica. Esta estrutura possui espaços de peso com dimensão infinita. Consideramos álgebras do tipo Borel diferentes das usuais, partindo de uma decomposição de $\mathfrak{g}$ não-usual. Esta álgebra é determinada por uma função $\varphi$ que atribui a cada inteiro um sinal + ou - .

Neste capítulo generalizamos os resultados obtidos em [San17b] e damos os critérios de irredutibilidade para módulos $\varphi$-Verma para álgebras de Krichever-Novikov hiperelípticas, obtendo que estas álgebras - como esperado - possuem módulos irredutíveis se e somente se possuem nível não nulo. 


\subsection{Módulos de Verma}

Nesta Seção, serão dadas definições para possibilitar a construção dos módulos de Verma. Seja g uma álgebra de Lie complexa, simples e com decomposição triangular

$$
\mathfrak{g}=\mathfrak{g}_{-} \oplus \mathfrak{h} \oplus \mathfrak{g}_{+},
$$

em que $\mathfrak{h}$ é uma álgebra de Lie abeliana. Dada uma representação $\pi: \mathfrak{h} \rightarrow \mathfrak{g l}(M)$ de $\mathfrak{h}$ num $\mathbb{C}$-espaço $M$, se $\phi \in \mathfrak{h}^{*}$, um vetor $v \in M$ é chamado vetor de peso, com peso $\phi$, se para todo $h \in \mathfrak{h}$ tivermos que

$$
\pi(h) v=\phi(h) v
$$

Utilizando estes objetos, podemos construir um submódulo gerado por $v$. Considere agora que $\pi$ é uma representação de $U(\mathfrak{g})$ em $M$. O espaço dado por

$$
\pi(U(\mathfrak{g})) v:=\{\pi(u) v \mid u \in U(\mathfrak{g})\}
$$

é o menor submódulo de $M$ que contém $v$ e é chamado de submódulo de $M$ gerado por $v$.

Estamos interessados em construir módulos de peso máximo, para isso é necessário definir o que é um vetor de peso máximo. Um vetor não nulo $v \in M$ é chamado vetor de peso máximo para $\mathfrak{g}$ se

1. $v$ é um vetor de peso em relação à ação de sua álgebra de Cartan $\mathfrak{h}$.

2. $\pi(x) v=0$ para todo $x \in \mathfrak{g}_{+}$.

Um g-módulo $M$ é dito um módulo de peso máximo se $M$ é gerado por um vetor $v$ de peso máximo. O peso $\lambda \in \mathfrak{h}^{*}$ do vetor de peso máximo $v$ é chamado peso máximo de $M$ e chamamos o par $(\lambda, v)$ um par de peso máximo de $M$.

Seja $M$ um módulo de peso máximo com vetor de peso máximo $v$ e peso máximo $\lambda$. Suponha que $\mathfrak{g}_{-}$tem base $\left\{x_{j}\right\}_{j \in J}$ de vetores de raízes indexados num conjunto totalmente ordenado $J$. Para cada $j \in J$ denotaremos por $\gamma_{j}$ a raiz correspondente a $x_{j}$, isto é $x_{j} \in \mathfrak{g}_{\gamma_{j}}$. Sabemos que

$$
x_{i j}^{n_{k}} \cdots x_{i_{1}}^{n_{1}} \cdot v
$$

com $k \geq 0, i_{j}>i_{j-1}>\cdots>i_{1}, n_{j} \in \mathbb{Z}_{>0}$ é um vetor de peso de $\mathrm{M}$ de peso $\lambda+$ $n_{1} \gamma_{1}+\cdots+n_{k} \gamma_{k}$ e esses vetores de peso geram como espaço vetorial $M$ o módulo de peso máximo $\mathrm{M}$.

Definição 5.1.1 Seja $\lambda \in \mathfrak{h}^{*}$. Um módulo de peso máximo $M(\lambda)$ para $\mathfrak{g}$ com peso máximo sendo o par $\left(\lambda, v_{+}\right)$é chamado módulo de Verma ${ }^{1}$ se para todo módulo de peso máximo $M$ com o par de peso máximo sendo $(\lambda, v)$ existe um homomorfismo de $\mathfrak{g}$-módulos $\eta: M(\lambda) \rightarrow$ $M$ tal que $v_{+} \mapsto v$.

Exemplo 5.1.2 Considere $x$, y e h base canônica de $\mathfrak{s l}(2 ; \mathbb{C})$ (veja Exemplo 2.1.6).

$$
x=\left(\begin{array}{ll}
0 & 1 \\
0 & 0
\end{array}\right), \quad y=\left(\begin{array}{cc}
0 & 0 \\
1 & 0
\end{array}\right), \quad h=\left(\begin{array}{cc}
1 & 0 \\
0 & -1
\end{array}\right) .
$$

\footnotetext{
${ }^{1}$ Daya-Nand Verma (1933 - 2012) foi um matemático indiano que apresentou a construção dos módulos que hoje recebem seu nome em sua tese de doutorado sob orientação de Nathan Jacobson na Universidade de Yale.
} 
com subálgebra de Cartan gerada por $h$. Seja $\lambda$ definido por $\lambda(h)=m$ em que $m$ é um número complexo arbitrário. O módulo de Verma com peso máximo $\lambda$ é gerado pelos vetores linearmente independentes $v_{0}, v_{1}, v_{2}, \ldots$ e a ação

$$
y \cdot v_{j}=v_{j+1} ; \quad x \cdot v_{j}=j(m-(j-1)) v_{j-1} ; \quad h \cdot v_{j}=(m-2 j) v_{j} .
$$

Exemplo 5.1.3 Seja $\mathfrak{a}=\mathfrak{a}_{-} \oplus \mathbb{C} c \oplus \mathfrak{a}_{+}$uma álgebra de Heisenberg (veja o Exemplo 2.1.7) com uma anti-involução $\sigma$ e bases $\left\{a_{j}\right\},\left\{\sigma\left(a_{j}\right)\right\}(j \in \mathbb{Z})$ para $\mathfrak{a}_{+} e \mathfrak{a}_{-}$tal que $\left[a_{j}, \sigma\left(a_{k}\right)\right]=$ $\delta_{j k} c, k \in \mathbb{Z}$. Seja $\alpha \in \mathfrak{h}^{*}$ e $M(\lambda)$ o módulo de Verma para a com peso máximo sendo $o$ par $\left(\lambda, v_{+}\right)$. Então $M(\lambda)=\bigoplus_{n \in \mathbb{N}} M(\lambda)^{\lambda-n \alpha}$ é a decomposição em espaços de peso correspondente de $M(\lambda)$. Além disso, se Â é o aniquilador de $v_{+}$, então $M(\lambda)=U\left(\mathfrak{a}_{-}\right)$. $v_{+} \hat{A}=U\left(\mathfrak{a}_{-}\right) \cdot v_{+}$.

Considere novamente $\mathfrak{g}$ uma álgebra de Lie afim e $\mathfrak{h}$ sua subálgebra de Cartan usual e $\mathfrak{z}=\mathbb{C} c$ o centro desta álgebra, em que $c$ é o elemento central canônico. Se $V$ é irredutível, então $c$ age como um escalar em $V$ e é chamado de nível de $V$.

\subsection{Módulos $\varphi$ Verma}

Podemos descrever $\mathfrak{g}$ como

$$
\mathfrak{g}=\mathfrak{h} \oplus \bigoplus_{\alpha \in \mathfrak{h}^{*} \backslash\{0\}} \mathfrak{g}_{\alpha}
$$

em que $\mathfrak{g}_{\alpha}=\{x \in \mathfrak{g} \mid[h, x]=\alpha(h) x, \forall h \in \mathfrak{h}\}$.Esta decomposição é denominada decomposição em espaços de raízes em relação a h (sua subálgebra de Cartan) (veja Subseção 2.3.1).

Apesar de na Subseção 2.3.1 ser apresentada uma definição de decomposição triangular de uma álgebra de Lie, há uma forma alternativa de obter tal decomposição. O conjunto $\Delta=\left\{\alpha \in \mathfrak{h}^{*} \mid \mathfrak{g}_{\alpha} \neq(0)\right\}$ é denominado sistema de raízes de $\mathfrak{g}$ e este conjunto tem uma partição $\Delta=\Delta_{+} \cup \Delta_{-}$em raízes positivas e negativas, em que $\Delta_{-}=-\Delta_{+}$. Caso $S \subseteq \Delta$ seja um conjunto tal que $S \cup(-S)=\Delta$ e que $S \cap(-S)=\varnothing$, diremos que $S$ é um conjunto que admite uma partição. Uma partição $\Delta=S \cup(-S)$ é dita uma partição fechada se sempre que $\alpha, \beta \in S$ e $\alpha+\beta \in \Delta$ tivermos que $\alpha+\beta \in S$. Para qualquer $S$ determinando uma partição de $\Delta$, os espaços $\mathfrak{g}_{S}=\oplus_{\alpha \in S} \mathfrak{g}_{\alpha}$ e $\mathfrak{g}_{-S}=\oplus_{\alpha \in-S} \mathfrak{g}_{\alpha}$ são subálgebras de $\mathfrak{g}$ e $\mathfrak{g}=\mathfrak{g}_{-S} \oplus \mathfrak{h} \oplus \mathfrak{g}_{S}$ é uma decomposição triangular de $\mathfrak{g}$.

\subsubsection{Módulos de Verma imaginários}

Seja $\Delta=S \cup(-S)$ uma partição fechada de $\Delta$. Pelo teorema de Poincaré-BirkhoffWitt (veja Teorema 2.1.12), a decomposição triangular $\mathfrak{g}=\mathfrak{g}_{-S} \oplus \mathfrak{h} \oplus \mathfrak{g}_{S}$ de $\mathfrak{g}$ determinada por $S$ fornece uma decomposição triangular da álgebra envelopante universal $U(\mathfrak{g})$ de $\mathfrak{g}$ dada por $U(\mathfrak{g})=U\left(\mathfrak{g}_{-S}\right) \otimes U(\mathfrak{h}) \otimes U\left(\mathfrak{g}_{S}\right)$. Considere $\mathfrak{b}_{S}=\mathfrak{h} \oplus \mathfrak{g}_{S}$ a álgebra de Borel associada. O funcional linear $\lambda \in \mathfrak{h}^{*}$ é estendido para a álgebra de Borel por um homomorfismo de álgebras também denotado $\lambda$ em $U(\mathfrak{h})$ e $U\left(\mathfrak{b}_{S}\right)$ com valores indo para zero em $\mathfrak{g}_{S}$. A cada $\lambda$ deste tipo, corresponde um $U\left(\mathfrak{b}_{S}\right)$ módulo um-dimensional $\mathbb{C} v$ em que $x v=\lambda(x) v$ para todo $x \in U\left(\mathfrak{b}_{S}\right)$. O módulo induzido

$$
M_{S}(\lambda)=U(\mathfrak{g}) \otimes_{U\left(\mathfrak{b}_{S}\right)} \mathbb{C} v,
$$

é um módulo do tipo Verma definido em [Cox94] e [FS93]. 


\subsubsection{Módulos $\varphi$ Verma para subálgebras de Heisenberg}

Dada uma matriz de Cartan do tipo afim $A=\left(a_{i j}\right)_{i, j=1}^{n}$ associada a $\mathfrak{g}$, podemos construir um diagrama de Dynkin de $A$ (ver Seção 2.3.2) . A partir do diagrama de Dynkin correspondente, considere

$$
\delta=\sum_{i=0}^{l} a_{i} \alpha_{i}
$$

em que os $a_{i}$ 's são exatamente as etiquetas dos vértices do diagrama e $\alpha_{i}$ são os elementos da base de raízes de g. O elemento $\delta$ é denominado raiz imaginária indivisível.

Se $\mathfrak{h}$ é subálgebra de Cartan de $\mathfrak{g}$, considere $\Pi=\left\{\alpha_{0}, \ldots, \alpha_{l}\right\}$ o seu conjunto de raízes simples e $\Pi^{\vee}=\left\{\alpha_{0}^{\vee}, \ldots, \alpha_{l}^{\vee}\right\}$ o seu conjunto de corraízes simples. O elemento

$$
c=\sum_{i=0}^{l} a_{i}^{\vee} \alpha_{i}^{\vee}
$$

é chamado elemento central canônico e até o final deste capítulo será denotado simplesmente como $c$.

Tomando $\Delta_{+}$o espaço das raízes positivas do sistema de raízes de $\mathfrak{g}$, podemos descrevê-lo como $\Delta_{+}=\Delta_{+}^{i m} \cup \Delta_{+}^{r e}$, sendo $\Delta_{+}^{i m}$ o espaço das raízes imaginárias, sabemos que $\Delta_{+}^{i m}=\left\{n \delta \mid n \in \mathbb{Z}_{>0}\right\}$.

O subespaço $L:=\mathbb{C} c \oplus \bigoplus_{n \in \mathbb{Z} \backslash\{0\}} \mathfrak{g}_{n \delta}$ forma uma subálgebra de Heisenberg da álgebra g. Então, $[x, y]=\zeta(x, y) c$ para $x \in \mathfrak{g}_{m \delta}, y \in \mathfrak{g}_{n \delta}$, em que $\zeta(x, y)$ é uma forma bilinear antissimétrica com $\zeta\left(\mathfrak{g}_{m \delta}, \mathfrak{g}_{n \delta}\right)=0$ para $n \neq-m$ e cuja restrição para $\mathfrak{g}_{m \delta} \times \mathfrak{g}_{n \delta}$ é não degenerada para todo $m \neq 0$. Podemos fazer a decomposição triangular de $L$ de forma que $L^{-} \oplus \mathbb{C} c \oplus L^{+}$, em que $L^{ \pm}=\bigoplus_{n \in \mathbb{N}} \mathfrak{g}_{ \pm n \delta}$.

Considere uma função $\varphi: \mathbb{N} \rightarrow\{ \pm\}$ e defina os espaços

$$
L_{\varphi}^{ \pm}=\left(\bigoplus_{n \in \mathbb{N}, \varphi(n)= \pm} \mathfrak{g}_{n \delta}\right) \oplus\left(\bigoplus_{m \in \mathbb{N}, \varphi(m)=\mp} \mathfrak{g}_{-m \delta}\right) .
$$

Estes espaços são subálgebras abelianas de $L \mathrm{e}$

$$
L=L_{\varphi}^{-} \oplus \mathbb{C} c \oplus L_{\varphi}^{+}
$$

é uma decomposição triangular de $L$.

Se $\mathbb{C} v$ é uma representação de dimensão 1 de $\mathbb{C} c \oplus L_{\varphi}^{+} \operatorname{com} c v=a v$ para algum escalar $a \in \mathbb{C}$ e $L_{\varphi}^{+} v=0$, definimos o módulo $\varphi$-Verma induzido por

$$
M_{\varphi}(a)=U(L) \otimes_{U\left(\mathrm{C} c \otimes L_{\varphi}^{+}\right)} \mathbb{C} v .
$$

Não é difícil perceber que quando $\varphi(n)=+, \forall n \in \mathbb{N}$, teremos a decomposição triangular usual de $L$ em (5.1) e o módulo de Verma de $L$ em (5.2) (compare com a Definição 5.1.1). A seguir, apresentaremos um critério de irredutibilidade para estes módulos obtido em [Bek+13].

Proposição 5.2.1 ([Bek+13], Proposição 3.3) $M_{\varphi}(a)$ é irredutível se, e somente se, $a \neq$ 0. 


\subsection{Módulos irredutíveis para subálgebras de Heisenberg}

Parte da motivação para o estudo de álgebras n-ponto, é a generalização da construção de álgebras laço. A Proposição 5.2.1 pode ser aplicada nas álgebras laço de $\mathfrak{s l}(2, \mathbb{C})$ de forma a obter módulos de $\varphi$-Verma irredutíveis (veja [Kac94]).

Seja $\mathcal{G}$ a álgebra laço de $\mathfrak{s l}(2, \mathbb{C})$ definida por $\mathcal{G}=\mathfrak{s l}(2, \mathbb{C}) \otimes \mathbb{C}\left[t, t^{-1}\right]$. Consideraremos uma extensão desta álgebra, $\hat{\mathfrak{G}}=\mathfrak{s l}(2, \mathbb{C}) \otimes \mathbb{C}\left[t, t^{-1}\right] \oplus \mathbb{C} k$, em que $\mathbb{C} k$ é uma álgebra de Lie de dimensão 1 e abeliana. $\mathrm{O}$ colchete de $\hat{\mathcal{G}}$ é dado por

$$
[f(t) \otimes x+\mu k, g(t) \otimes y+v k]=f(t) g(t) \otimes[x, y]_{\mathfrak{g}}+\phi(f(t) \otimes x, g(t) \otimes y) k
$$

em que $\phi$ é uma função $\phi: \mathcal{G} \times \mathcal{G} \rightarrow \mathbb{C}$ tal que

$$
\phi(f(t) \otimes x, g(t) \otimes y):=\left(t \frac{d}{d t} f(t) \otimes x, g(t) \otimes y\right)
$$

para $f, g \in \mathbb{C}\left[t, t^{-1}\right]$ e $x, y \in \mathfrak{s l}(2, \mathbb{C})$.

A subálgebra de Heisenberg de $\hat{\mathfrak{G}}$ é $L:=\mathfrak{h} \otimes \mathbb{C}\left[t, t^{-1}\right] \oplus \mathbb{C} k$, em que $\mathfrak{h}$ é a subálgebra de Cartan de $\mathfrak{s l}(2, \mathbb{C})$. Considere a seguinte álgebra de Heisenberg dada pelo espaço $H=\mathbb{C} k \oplus \bigoplus_{i \in \mathbb{Z} \backslash\{0\}} \mathbb{C} e_{i}$, em que $e_{i}$ são geradores tais que $\left[e_{i}, e_{j}\right]=\delta_{i,-j} k$, e $\left[e_{j}, k\right]=0$ para todo $i \geq 1$ e todo $j$. A subálgebra de Heisenberg $L$, da Seção 5.2, pode ser descrita como $H$ escolhendo uma base ortogonal em cada espaço de raízes $g_{k \delta}$ e uma base dual em $\mathfrak{g}_{-k \delta}$ para cada $j \geq 1$.

Sabemos que $\mathfrak{h}$, a álgebra de Cartan $\operatorname{de} \mathfrak{s l}(2, \mathbb{C})$, tem dimensão 1. Seja

$$
\varphi:\left(\mathfrak{h} \otimes \mathbb{C}\left[t, t^{-1}\right] \oplus \mathbb{C} k\right) \mapsto\left(\mathbb{C} k \oplus \bigoplus_{i \in \mathbb{Z} \backslash\{0\}} \mathbb{C} e_{i}\right)
$$

aplicação linear tal que

$$
\varphi\left(h t^{n}+a\right)=a+e_{n}
$$

em que $h \in \mathfrak{h}$ e $a \in \mathbb{C} k$. Como $\varphi$ define um isomorfismo, podemos diretamente aplicar a Proposição 5.2.1 obtendo assim módulos de Verma e $\varphi$-Verma irredutíveis para essa extensão de álgebra laço para $\mathfrak{s l}(2, \mathbb{C})$.

\subsection{A álgebra de Lie hiperelíptica}

A álgebra de Lie hiperelíptica é uma família de álgebras de Krichever-Novikov superelípticas e já foi apresentada na Subseção 3.7.4, no entanto, cabe aqui retomar as principais definições e resultados para formular de forma prática os objetivos centrais deste capítulo sem que o leitor tenha de recorrer a sucessivas consultas às definições e resultados no Capítulo 3.

Nesta seção estudaremos a extensão central universal da álgebra de Lie $\mathfrak{s l}(2, \mathbb{C})$ $\otimes R$ em que $R=\mathbb{C}\left[t^{ \pm 1}, u: u^{2}=p(t)\right], \operatorname{com} p(t)=t\left(t-\alpha_{1}\right) \cdots\left(t-\alpha_{r}\right)=\sum_{i=1}^{r+1} a_{i} t^{i} \in$ $\mathbb{C}[t], \alpha_{i}{ }^{\prime}$ s são números complexos não nulos dois a dois distintos e $a_{r+1}=1$. Usando o Teorema 3.7.6, é possível descrever a extensão central universal de $\mathfrak{s l}(2, \mathbb{C}) \otimes R$.

As relações de comutatividade de $\hat{\mathfrak{g}}=(\mathfrak{g} \otimes R) \oplus \Omega_{R}^{1} / d R$ são

$$
\begin{aligned}
& {[x \otimes f, y \otimes g]=[x y] \otimes f g+(x, y) \overline{f d g} \mathrm{e}} \\
& {[x \otimes f, \omega]=0,}
\end{aligned}
$$

em que $x, y \in \mathfrak{g}, f, g \in R, \omega \in \Omega_{R}^{1} / d R,(\cdot, \cdot)$ denota a forma de Killing de $\mathfrak{g}$. 
O Teorema 3.7.20 nos garante que, neste caso, $\left\{\overline{t^{-1} d t}, \overline{t^{-1} u d t}, \ldots, \overline{t^{-r} u d t}\right\}$ é uma base para $\Omega_{R}^{1} / d R$.

Fixemos

$$
\omega_{0}:=\overline{t^{-1} d t} \quad \text { e } \quad \omega_{k}:=\overline{t^{-k} u d t}
$$

em que $1 \leq k \leq r$

Definiremos famílias de polinômios que possibilitam descrever $\hat{\mathfrak{g}}$ em termos de suas relações de comutatividade. Considere $m=2$ e $a_{0}=0$, definemos $P_{k, i}:=$ $P_{k, i}\left(a_{1}, \ldots, a_{r}\right), k \geq-r,-r \leq i \leq-1$ polinômios em $a_{i}$ satisfazendo as seguintes relações de recursividade:

$$
(2 k+r+3) P_{k, i}=-\sum_{j=1}^{r}(3 j+2 k-2 r) a_{j} P_{k-r+j-1, i}
$$

para $k \geq 0$ com a condição inicial $P_{l, i}=\delta_{l, i},-r \leq i, l \leq-1$. Além disso, definiremos $Q_{m, i}$ satisfazendo

$$
(2 m-3) a_{1} Q_{m, i}=\left(\sum_{j=2}^{r+1}(3 j-2 m) a_{j} Q_{m-j+1, i}\right)
$$

com as condições iniciais $Q_{m, i}=\delta_{m,-i}$ para $1 \leq m \leq r \mathrm{e}-r \leq i \leq-1$.

Teorema 5.4.1 ([CI18], Teorema 5.1) Seja $a_{1} \neq 0$. Seja $\mathfrak{g}$ uma álgebra de Lie simples de dimensão finita sobre os números complexos com forma de Killing $(\cdot, \cdot)$ e para $a=$ $\left(a_{1}, \ldots, a_{r}\right)$ defina $\psi_{i j}(a) \in \Omega_{R}^{1} / d R$ por

$$
\psi_{i j}(a)= \begin{cases}\sum_{k=1}^{r} P_{i+j-1,-k} \omega_{k} & \text { se } i+j \geq-r+1, \\ \sum_{k=1}^{r} Q_{-i-j+1,-k} \omega_{k} & \text { caso contrário. }\end{cases}
$$

A extensão central universal da álgebra de Lie hiperelíptica $\mathfrak{g} \otimes R$ é um álgebra de Lie $\mathbb{Z} / 2 \mathbb{Z}$ graduada

$$
\hat{\mathfrak{g}}=\hat{\mathfrak{g}}^{0} \oplus \hat{\mathfrak{g}}^{1}
$$

em que

$$
\hat{\mathfrak{g}}^{0}=\left(\mathfrak{g} \otimes \mathbb{C}\left[t, t^{-1}\right]\right) \oplus \mathbb{C} \omega_{0}, \quad \hat{\mathfrak{g}}^{1}=\left(\mathfrak{g} \otimes \mathbb{C}\left[t, t^{-1}\right] u\right) \oplus \bigoplus_{k=1}^{r}\left(\mathbb{C} \omega_{k}\right)
$$

com colchetes dados por

$$
\begin{aligned}
{\left[x \otimes t^{i}, y \otimes t^{j}\right] } & =[x, y] t^{i+j}+\delta_{i+j, 0} j(x, y) \omega_{0} \\
{\left[x \otimes t^{i} u, y \otimes t^{j} u\right] } & =[x, y] \otimes t^{i+j} p(t)+\sum_{k=1}^{r+1}\left(j+\frac{1}{2} k\right) a_{k} \delta_{i+j,-k} \omega_{0} \\
{\left[x \otimes t^{i} u, y \otimes t^{j}\right] } & =[x, y] u \otimes t^{i+j} u+j(x, y) \psi_{i j}(a) .
\end{aligned}
$$

Esta álgebra é denominada álgebra de Lie hiperelíptica para o caso $\mathfrak{s l}(2, \mathbb{C})$. 


\subsection{Subálgebra de Heisenberg hiperelíptica}

Seja $\mathfrak{h}$ a subálgebra de Cartan de $\mathfrak{s l}(2, \mathbb{C})$. A álgebra $\mathfrak{s l}(2, \mathbb{C}) \otimes R$, é a subálgebra de Heisenberg de $\hat{\mathfrak{g}}$. A álgebra de Lie com geradores $b_{m}, b_{m}^{1}, m \in \mathbb{Z}, 1_{i}, i \in\{0,1, \ldots, r\}$ e relações

$$
\begin{aligned}
& {\left[b_{m}, b_{n}\right]=2 n \delta_{m+n, 0} 1_{0},} \\
& {\left[b_{m}^{1}, b_{n}^{1}\right]=(1 / 2)(n-m) a_{-(m+n)} 1_{0},} \\
& {\left[b_{m}^{1}, b_{n}\right]=2 n \sum_{k=1}^{r}\left(\left(\delta_{m+n-1 \geq-r}\right) P_{m+n-1,-k}+\left(\delta_{m+n-1<-r}\right) Q_{-m-n+1,-k}\right) 1_{k},} \\
& {\left[b_{m}, 1_{i}\right]=\left[b_{m}^{1}, 1_{i}\right]=\left[1_{i}, 1_{j}\right]=0,}
\end{aligned}
$$

em que $i, j \in\{0, \ldots, r\}$ e $k \in \mathbb{Z}$ é denominada álgebra de Heisenberg hiperelíptica e será denotada por $\hat{\mathfrak{h}}$.

Seja $\varphi$ uma função de $\mathbb{Z}$ em $\{ \pm\}$ tal que $\varphi(n)=+\Leftrightarrow \varphi(-n)=-$. Considere

$$
\hat{\mathfrak{h}}_{\varphi}^{ \pm}=\left(\sum_{\substack{n \in \mathbb{Z}_{<0} \\ \varphi(n)=\mp}}\left(\mathbb{C} b_{n}+\mathbb{C} b_{n}^{1}\right)\right) \oplus\left(\sum_{\substack{m \in \mathbb{Z}_{>0} \\ \varphi(m)= \pm}}\left(\mathbb{C} b_{m}+\mathbb{C} b_{m}^{1}\right)\right)
$$

$\mathrm{e}$

$$
\hat{\mathfrak{h}}_{0}=\mathbb{C} b_{0}+\mathrm{C} b_{0}^{1}+\sum_{i=0}^{r} 1_{i} \mathbb{C} .
$$

Como consequência das relações definidas em (5.4), (5.5), (5.6) e (5.7), temos que $\hat{\mathfrak{b}}_{\varphi}:=\hat{\mathfrak{h}}^{0} \oplus \hat{\mathfrak{h}}_{\varphi}^{+}$é uma subálgebra do tipo Borel.

Lema 5.5.1 Seja $\mathcal{V}=\mathbb{C} v_{0} \oplus \mathbb{C} v_{1}$ uma representação 2 dimensional de $\hat{\mathfrak{b}}$, em que $\hat{\mathfrak{h}}_{\varphi}^{+} v_{i}=0$ para $i=0,1$. Suponha que $\lambda, \mu, v, \chi_{j}, \gamma, \kappa_{0} \in \mathbb{C}$ para $j \in\{1, \ldots, r\}$ são tais que

$$
\begin{aligned}
& b_{0} \cdot v_{0}=\lambda v_{0} \quad, \quad b_{0}^{1} \cdot v_{0}=\mu v_{0}+v v_{1} \quad, \quad 1_{j} v_{i}=\chi_{j} v_{i}, \\
& b_{0} \cdot v_{1}=\lambda v_{1}, \quad b_{0}^{1} \cdot v_{1}=\gamma v_{0}+\mu v_{1}, \quad 1_{0} v_{i}=\kappa_{0} v_{i} \text {, }
\end{aligned}
$$

para $i \in\{0,1\}$. Então,

$$
\begin{aligned}
\sum_{k=1}^{r}\left(P_{m+n-1,-k}\right) \chi_{k} & =0, \text { se } m+n \geq-r+1, e \\
\sum_{k=1}^{r}\left(Q_{-m-n+1,-k}\right) \chi_{k} & =0 \text { caso contrário. }
\end{aligned}
$$

Demonstração: Como $b_{m}$ age como multiplicação por um escalar para $m, n \in \mathbb{Z}$, a primeira relação (5.4) está satisfeita. A segunda relação (5.5) também está satisfeita. Caso $n=0$, então como $b_{0}$ age como multiplicação por escalar, a relação (5.6) não implica em uma condição para $\lambda, \mu, v, \chi_{j}, \gamma, \kappa_{0} \in \mathbb{C}$, a terceira relação implica uma condição sobre $\chi_{j}$ de forma que

$$
\begin{aligned}
0 & =b_{m}^{1} b_{n} v_{i}-b_{n} b_{m}^{1} v_{i}=\left[b_{m}^{1}, b_{n}\right] v \\
& =2 n \sum_{k=1}^{r}\left(\left(\delta_{m+n \geq-r+1}\right) P_{m+n-1,-k}+\left(\delta_{m+n<-r+1}\right) Q_{-m-n+1,-k}\right) 1_{k} v
\end{aligned}
$$




\subsection{Módulos $\varphi$ Verma para a subálgebra de Heisenberg hipe- relíptica}

Considere o seguinte $\hat{\mathfrak{g}}$-módulo $\varphi$-Verma induzido

$$
M_{\hat{\mathfrak{g}}, \varphi}=U(\hat{\mathfrak{g}}) \otimes_{\hat{\mathfrak{b}}_{\varphi}} \mathbb{C} v .
$$

Dado um inteiro $n$, considere que

$$
\operatorname{sgn}(n)= \begin{cases}+ & \text { se } n>0, \\ - & \text { se } n<0 .\end{cases}
$$

Lema 5.6.1 Se $m, n \in \mathbb{Z} \backslash\{0\}, \varphi(m)=\operatorname{sgn}(m)$ e $\varphi(n) \neq \operatorname{sgn}(n)$, então

$$
\begin{aligned}
& b_{m}\left(b_{n}\right)^{l_{v}}=\left(2 \ln \kappa_{0}\right) \delta_{m+n, 0}\left(\left(b_{n}\right)^{l-1}\right) v, \\
& b_{m}\left(b_{n}^{1}\right)^{l_{v}}=0, \\
& b_{m}^{1}\left(b_{n}^{1}\right)^{l_{v}}=(1 / 2)\left(l \kappa_{0}\right)\left((n-m) a_{-(m+n)}\right)\left(\left(b_{n}^{1}\right)^{l-1}\right) v, \\
& b_{m}^{1}\left(b_{n}\right)^{l} v=0 .
\end{aligned}
$$

Demonstração: Usando o Lema 5.5.1 e as relações que definem a subálgebra de Heisenberg hiperelíptica (veja a Seção 5.5) temos que

$$
\begin{aligned}
b_{m}\left(b_{n}\right)^{l} v & =\left[b_{m},\left(b_{n}\right)^{l}\right] v+\left(b_{n}\right)^{l} b_{m v} \\
& =l\left(b_{n}\right)^{l-1}\left[b_{m}, b_{n}\right] v \\
& =\left(2 l n \kappa_{0}\right) \delta_{m+n, 0}\left(\left(b_{n}\right)^{l-1}\right) v . \\
b_{m}\left(b_{n}^{1}\right)^{l} v & =\left[b_{m},\left(b_{n}^{1}\right)^{l}\right] v+\left(b_{n}^{1}\right)^{l} b_{m} v \\
& =l\left(b_{n}^{1}\right)^{l-1}\left[b_{m}, b_{n}^{1}\right] v \\
& =0 \\
b_{m}^{1}\left(b_{n}^{1}\right)^{l} v & =\left[b_{m}^{1},\left(b_{n}^{1}\right)^{l}\right] v+\left(b_{n}^{1}\right)^{l} b_{m}^{1} v \\
& =l\left(b_{n}^{1}\right)^{l-1}\left[b_{m}^{1}, b_{n}^{1}\right] v \\
& =(1 / 2)\left(l \kappa_{0}\right)\left((n-m) a_{-(m+n)}\right)\left(\left(b_{n}^{1}\right)^{l-1}\right) v . \\
b_{m}^{1}\left(b_{n}\right)^{l} & =\left[b_{m}^{1},\left(b_{n}\right)^{l}\right] v+\left(b_{n}\right)^{l} b_{m}^{1} v \\
& =l\left(b_{n}\right)^{l-1}\left[b_{m}^{1}, b_{n}\right] v \\
& =0
\end{aligned}
$$

Considerando os resultados alcançados até aqui, podemos demonstrar um critério de irredutibilidade para o módulo $M_{\hat{\mathfrak{g}}, \varphi}$.

Teorema 5.6.2 $M_{\hat{\mathfrak{g}}, \varphi}$ é irredutível se, e somente se, $\kappa_{0} \neq 0$. 
Demonstração: Defina

$$
\begin{array}{r}
\left(M_{\hat{\mathfrak{g}}, \varphi}\right)_{n}=\left\{w \in M_{\hat{\mathfrak{g}}, \varphi}: w=\left(\sum_{(\bar{\alpha}, \bar{\beta})} \xi(\bar{\alpha}, \bar{\beta}) \prod_{\substack{i \in \mathbb{Z} \backslash\{0\} \\
\varphi(i) \neq \operatorname{sgn}(i)}} b_{i}^{\alpha_{i}} \prod_{\substack{i \in \mathbb{Z} \backslash\{0\} \\
\varphi(j) \neq \operatorname{sgn}(n)}}\left(b_{j}^{1}\right)^{\beta_{j}}\right) v,\right. \\
\left.\sum_{i} \alpha_{i}+\sum_{j} \beta_{j}=n, \forall(\bar{\alpha}, \bar{\beta})\right\} .
\end{array}
$$

Dizemos que $\operatorname{deg}(w)=n$ se $w \in\left(M_{\hat{\mathfrak{g}}, \varphi}\right)_{n}$. Suponha que $\kappa_{0} \neq 0$ e procedamos por indução em $\operatorname{deg}(w)$.

1. Suponha que $\operatorname{deg}(w)=1$, então

$$
w=\left(\sum_{\substack{i \in \mathbb{Z} \\ \varphi(i) \neq \operatorname{sgn}(i)}} \xi_{i} b_{i}+\sum_{\substack{i \in \mathbb{Z} \\ \varphi(i) \neq \operatorname{sgn}(i)}} \xi_{i}^{1} b_{i}^{1}\right) v
$$

em que apenas uma quantidade finita de $\xi_{i}, \xi_{i}^{1} \in \mathbb{C}$ são não nulos.

(a) Se $\xi_{i}^{\prime} s$ são todos nulos, então existe $\xi_{m}^{1} \neq 0$ para algum $m \in \mathbb{Z}$ tal que $\varphi(m) \neq \operatorname{sgn}(m)$. Seja $x \in \hat{\mathfrak{h}}_{\varphi}^{+}$tal que $x=b_{-m}^{1}$. Temos que

$$
x w=x \sum_{\substack{i \in \mathbb{Z} \\ \varphi(i) \neq \operatorname{sgn}(i)}} \xi_{i}^{1} b_{i}^{1} v=(1 / 2) \kappa_{0} \sum_{\substack{k \in \mathbb{Z} \\ \varphi(i) \neq \operatorname{sgn}(i)}} \xi_{k}^{1}(k-m) a_{-(k+m)} v .
$$

(b) Se há algum $\xi_{k}$ não nulo, seja $x \in \hat{\mathfrak{h}}_{\varphi}^{+}$tal que $x=b_{-k}$. Teremos que

$$
x w=x \sum_{\substack{i \in \mathbb{Z} \\ \varphi(i) \neq \operatorname{sgn}(i)}} \xi_{i} b_{i} v=2 \kappa_{0} k \xi_{k} v
$$

Portanto, em ambos os casos, temos que existe $x \in \hat{\mathfrak{h}}_{\varphi}^{+}$tal que $x w \neq 0$ e $\operatorname{deg}(x w)=0$.

2. Suponha que para todo $v \in M_{\hat{\mathfrak{g}}, \varphi}$ com grau $n$, existe $y \in \hat{\mathfrak{h}}^{+}$tal que $y w \neq 0$ e $\operatorname{deg}(y w)=0$. Suponha também que $\operatorname{deg}(w)=n+1$, assim um elemento arbitrário $M_{\hat{\mathfrak{g}}, \varphi}$ com este grau pode ser descrito como

$$
\begin{aligned}
w= & \left(\sum_{\substack{i \in \mathbb{Z} \\
\varphi(i) \neq \operatorname{sgn}(i)}} \xi_{i} b_{i}^{(n+1)}+\sum_{\substack{i \in \mathbb{Z} \\
\varphi(i) \neq \operatorname{sgn}(i)}} \xi_{i}^{1}\left(b_{i}^{1}\right)^{(n+1)}\right. \\
& \left.+\sum_{(\bar{\alpha}, \bar{\beta})} \xi(\bar{\alpha}, \bar{\beta}) \prod_{\substack{i \in \mathbb{Z} \\
\varphi(i) \neq \operatorname{sgn}(i)}} b_{i}^{\alpha_{i}} \prod_{\substack{j \in \mathbb{Z} \\
\varphi(j) \neq \operatorname{sgn}(j)}}\left(b_{j}^{1}\right)^{\beta_{j}}\right) v
\end{aligned}
$$

em que, $\bar{a}:=\left\{a_{0}, a_{2}, \ldots, a_{l}\right\}$ com $a_{i} \in \mathbb{Z}$ e de forma que apenas uma quantidade finita de $a_{i}, \xi_{i}, \xi_{i}^{1}$ e $\xi(\bar{\alpha}, \bar{\beta})$ são não nulos. 
(a) Caso haja $\xi(\bar{\alpha}, \bar{\beta}) \neq 0$, então seja $\xi(\bar{k}, \bar{l}) \neq 0$ e considere $m$ o maior índice com $k_{m} \neq 0$ e $x \in \hat{\mathfrak{h}}_{\varphi}^{+}$tal que $x=b_{-m}$. Além disso, reorganize os monômios de forma que, com as constantes apropriadas,

$$
\begin{aligned}
& w=\left(\sum_{\substack{i \in \mathbb{Z} \backslash\{0\} \\
\varphi(i) \neq \operatorname{sgn}(i)}} \xi_{i} b_{i}^{(n+1)}+\sum_{\substack{i \in \mathbb{Z} \backslash\{0\} \\
\varphi(i) \neq \operatorname{sgn}(i)}} \xi_{i}^{1}\left(b_{i}^{1}\right)^{(n+1)}\right. \\
& \left.+\sum_{(\bar{\alpha}, \bar{\beta})} \xi(\bar{\alpha}, \bar{\beta}) \prod_{\substack{i \in \mathbb{Z} \backslash\{0\} \\
\varphi(i) \neq \operatorname{sgn}(i)}} b_{i}^{\alpha_{i}} \prod_{\substack{i \in \mathbb{Z} \backslash\{0\} \\
\varphi(i) \neq \operatorname{sgn}(i)}}\left(b_{j}^{1}\right)^{\beta_{j}}\right) v \\
& =\left(\sum_{\substack{i \in \mathbb{Z} \backslash\{0\} \\
\varphi(i) \neq \operatorname{sgn}(i)}} \tilde{\xi}_{i} b_{i}^{(n+1)}+\sum_{\substack{i \in \mathbb{Z} \backslash\{0\} \\
\varphi(i) \neq \operatorname{sgn}(i)}} \tilde{\xi}_{i}^{1}\left(b_{i}^{1}\right)^{(n+1)}\right. \\
& +\sum_{(\bar{\alpha}, \bar{\beta})} \tilde{\xi}(\bar{\alpha}, \bar{\beta}) b_{m}^{\alpha_{m}} \prod_{\substack{i \in \mathbb{Z} \backslash\{0, m\} \\
\varphi(i) \neq \operatorname{sgn}(i)}} b_{i}^{\alpha_{i}} \prod_{\substack{j \in \mathbb{Z} \backslash\{0\} \\
\varphi(j) \neq \operatorname{sgn}(j)}}\left(b_{j}^{1}\right)^{\beta_{j}} \\
& \left.+\sum_{(\bar{\alpha}, \bar{\beta})} \tilde{\xi}(\bar{\alpha}, \bar{\beta}) b_{i_{0}}^{\alpha_{i_{0}}} \prod_{\substack{i \in \mathbb{Z} \backslash\{0, m\} \\
\varphi(i) \neq \operatorname{sgn}(i)}} b_{i}^{\alpha_{i}} \prod_{\substack{j \in \mathbb{Z} \backslash\{0\} \\
\varphi(j) \neq \operatorname{sgn}(j)}}\left(b_{j}^{1}\right)^{\beta_{j}}\right) v .
\end{aligned}
$$

Aplicando $x$ em $w$ teremos que

$$
\begin{aligned}
x w= & \tilde{\xi}_{m} 2 \kappa_{0}(n+1) m b_{m}^{n} v+0 \\
& +\sum_{(\bar{\alpha}, \bar{\beta})} \tilde{\xi}(\bar{\alpha}, \bar{\beta}) b_{-m} b_{m}^{\alpha_{m}} \prod_{\substack{i \in \mathbb{Z} \backslash\{0, m\} \\
\varphi(i) \neq \operatorname{sgn}(i)}} b_{i}^{\alpha_{i}} \prod_{\substack{i \in \mathbb{Z} \backslash\{0\} \\
\varphi(i) \neq \operatorname{sgn}(i)}}\left(b_{j}^{1}\right)^{\beta_{j}} v \\
& +\sum_{(\bar{\alpha}, \bar{\beta})} \tilde{\xi} b_{-m} b_{i_{0}}^{\alpha_{i_{0}}}(\bar{\alpha}, \bar{\beta}) b_{m}^{\alpha_{m}} \prod_{\substack{i \in \mathbb{Z} \backslash\{0, m\} \\
\varphi(i) \neq \operatorname{sgn}(i)}} b_{i}^{\alpha_{i}} \prod_{\substack{j \in \mathbb{Z} \backslash\{0\} \\
\varphi(j) \neq \operatorname{sgn}(j)}}\left(b_{j}^{1}\right)^{\beta_{j} v} \\
= & \tilde{\xi}_{m} 2 \kappa_{0}(n+1) m b_{m}^{n} v \\
& +\sum_{(\bar{\alpha}, \bar{\beta})} \tilde{\xi}(\bar{\alpha}, \bar{\beta}) \alpha_{m}\left(2 m \kappa_{0}\right) b_{m}^{\alpha_{m}-1} \prod_{\substack{i \in \mathbb{Z} \backslash\{0, m\} \\
\varphi(i) \neq \operatorname{sgn}(i)}} b_{i}^{\alpha_{i}} \prod_{\substack{j \in \mathbb{Z} \backslash\{0\} \\
\varphi(j) \neq \operatorname{sgn}(j)}}\left(b_{j}^{1}\right)^{\beta_{j}} v .
\end{aligned}
$$

Então $x w \neq 0$ e $\operatorname{deg}(x w)=n$.

(b) $\operatorname{Caso} \xi(\bar{\alpha}, \bar{\beta})=0, \forall(\bar{\alpha}, \bar{\beta})$.

(b.i) Se existe $\xi_{k} \neq 0$ então seja $x=b_{-k}$ e $x w=2 \kappa_{0}(n+1) k \xi_{k}^{n} b_{k}^{n} v$.

(b.ii) Se todos os $\xi_{i}$ 's são iguais a zero, então eixste um $\chi_{m}^{1}$ para algum $m \in \mathbb{Z}$ tal que $\varphi(m) \neq \operatorname{sgn}(m)$. Seja $x=b_{-m}^{1}$. Teremos que

$$
x w=(1 / 2)(n+1) \kappa_{0} \sum_{\substack{k \in \mathbb{Z} \\ \varphi(i) \neq \operatorname{sgn}(i)}} \xi_{k}^{1}(k-m) a_{-(k+m)}\left(b_{k}\right)^{n} v .
$$

Portanto $x w \neq 0$ e $\operatorname{deg}(x w)=n$.

Como $\kappa_{0} \neq 0$ temos que o submódulo gerado por $w$ contém $v$ e consequentemente é todo $M_{\hat{\mathfrak{g}}, \varphi}$. Entretanto $w$ é um elemento arbitrário não nulo, consequentemente $M_{\hat{\mathfrak{g}}, \varphi}$ é um módulo irredutível, neste caso.

Caso $\kappa_{0}=0$ então $N_{\hat{\mathfrak{g}}, \varphi}:=\bigoplus_{n \in \mathbb{Z} \backslash\{0\}}\left(M_{\hat{\mathfrak{g}}, \varphi}\right)_{n}$ é um submódulo próprio. 


\section{Capítulo 6}

\section{Perspectivas futuras e considerações finais}

Durante o desenvolvimento deste trabalho, foram estudados essencialmente três aspectos das álgebras de Krichever-Novikov:

1. A extensão central universal de álgebras de Krichever-Novikov superelípticas;

2. Famílias de polinômios ortogonais não-clássicos satisfazendo determinadas equações diferenciais de ordem maior que 2 que surgem como coeficientes nas relações de comutatividade de álgebras de Krichever-Novikov superelípticas;

3. Módulos $\varphi$-Verma irredutíveis para subálgebras de Heisenberg de álgebras de Krichever-Novikov hiperelípticas.

Este estudo foi motivado pelas perguntas enunciadas na Introdução:

Pergunta 1: A teoria desenvolvida para curvas hiperelípticas pode ser estendida para o caso das curvas superelípticas?

Pergunta 2: À partir das famílias de polinômios obtidas durante o estudo das relações de comutatividade de álgebras de Krichever-Novikov do tipo superelípticas, é possível obter famílias de polinômios ortogonais não-clássicos que satisfazem equações diferenciais de ordem maior que 2 ?

Pergunta 3: Quais são os critérios de irredutibilidade para módulos $\varphi$-Verma de subálgebras de Heisenberg hiperelípticas?

O objetivo deste capítulo é apresentar perspectivas futuras de pesquisa nessa área e apresentar considerações finais deste trabalho.

\subsection{Perspectivas futuras}

Os resultados obtidos durante o desenvolvimento desta tese nos leva a algumas questões naturais que podem ser investigadas em oportunidade futura e forma organizadas nas perguntas a seguir.

Pergunta 4: Partindo do Teorema 3.8.10 é possível estudar realizações de álgebras de Krichever-Novikov superelípticas do tipo $\mathfrak{s l}(2, \mathbb{C}) \otimes R \oplus \Omega_{R} / d R$ aplicando as mesmas estratégias vistas em [CJ14] e [Cox08]? 
Pergunta 5: Utilizando a estratégia aplicada na Seção 4.3 é possível obter famílias de polinômios ortogonais não-clássicos que satisfazem determinadas equações diferenciais de ordem maior que 2 considerando curvas do tipo $u^{m}=t^{4}-2 c t^{2}+1$ com $m \geq 2$ ?

Pergunta 6: Há outras famílias de polinômios do tipo $u^{m}=p(t)$ que podem fornecer famílias de polinômios ortogonais não-clássicos que satisfazem determinadas equações diferenciais de ordem maior que 2 utilizando as relações de recursividade advindas de álgebras de Krichever-Novikov superelípticas?

Pergunta 7: No Capítulo 5 são estabelecidos critérios de irredutibilidade para módulos $\varphi$ Verma para subálgebras de Heisenberg hiperelípticas. É possível estender esta estratégias para módulos sobre álgebras de Krichever-Novikov superelípticas em geral?

A tese apresentada, motiva-me a conjecturar que as perguntas acima podem ser respondidas de forma afirmativa. Neste caso, conseguiríamos responder à Pergunta 1 (neste contexto) mostrando que o que já foi desenvolvido para álgebras de Krichever-Novikov no caso hiperelíptico pode ser estendido para o caso superelíptico geral.

\subsection{Considerações finais}

Este trabalho introduz três resultados principais distribuídos em três capítulos. Os resultados apresentados no Capítulo 3, são completamente vinculados ao aspecto geométrico das estruturas algébricas em questão. Só é possível assegurar-se de que o conjunto gerador encontrado é uma base, pois a dimensão do espaço é conhecida graças à propriedades geométricas. O que é apresentado no Capítulo 4 conecta os resultados algébricos obtidos à partir de propriedades geométricas com funções especiais e equações diferenciais, confirmando a potência deste tema de pesquisa como conexão entre diferentes áreas da Matemática. Por último, o estudo do critério de irredutibilidade para módulos de subálgebras de Heisenberg de álgebras hiperelípticas feito no Capítulo 5, apresenta como os resultados obtidos anteriormente para o caso hiperelíptico ainda oferecem desdobramentos novos. Todos estas descobertas podem ser conectadas e, no futuro, aprofundadas levando em consideração as perguntas apresentadas na seção anterior.

As álgebras de Krichever-Novikov continuam a despertar grande interesse dos físicos pelas suas aplicações, no entanto, estas estruturas também são muito importantes do ponto de vista da Matemática Pura pois fornecem uma grande quantidade de álgebras de Lie de dimensão infinita que são "controláveis"pela sua natureza geométrica. Esta característica nos motiva a continuar o estudo destas estruturas conectando os achados com diversas áreas do conhecimento matemático, como já vem sendo feito nas últimas décadas. 


\section{Bibliografia}

[Bek+13] Viktor Bekkert et al. "New irreducible modules for Heisenberg and affine Lie algebras". Em: Journal of Algebra 373 (2013), pp. 284-298. ISSN: 00218693. DOI: $10.1016 / \mathrm{j} \cdot \mathrm{j}$ algebra.2012.09.035.

[Bre94] Murray Bremner. "Universal central extensions of elliptic affine Lie algebras". Em: Journal of Mathematical Physics 35.12 (1994), pp. 6685-6692. DOI: $10.1063 / 1.530700$.

[Bre95] Murray Bremner. "Four-point affine Lie algebras". Em: Proceedings of the American Mathematical Society 123.7 (1995), pp. 1981-1989. DOI: 10.1090/ S0002-9939-1995-1249871-8.

[BSZ15] Lubjana Beshaj, Tony Shaska, Eustrat Zhupa. Advances on Superelliptic Curves and their Applications. Netherlands: IOS Press BV, 2015. ISBN: 9781-61499-520-3.

[CF11] Ben Cox, Vyacheslav Futorny. "DJKM algebras I: Their universal central extension". Em: Proceedings of the American Mathematical Society 139.10 (2011), pp. 3451-3451. ISSN: 0002-9939. DOI: 10 . 1090 / S0002 - 9939 2011-10906-7.

[CFM14] Ben Cox, Vyacheslav Futorny, Renato Alessandro Martins. "Free field realizations of the Date-Jimbo-Kashiwara-Miwa algebra". Em: Developments in Mathematics 38 (2014), pp. 111-136. ISSN: 13892177. DOI: 0 . 1007/978-3-319-09804-3_5.

[CFT13] Ben Cox, Vyacheslav Futorny, Juan A. Tirao. "DJKM algebras and nonclassical orthogonal polynomials". Em: Journal of Differential Equations 255.9 (2013), pp. 2846-2870. DOI: 10.1016/j . jde.2013.07.020.

[Chi01] Theodore Seio Chihara. "45 years of orthogonal polynomials: a view from the wings". Em: Journal of Computational and Applied Mathematics 133.1-2 (2001). ISSN: 03770427. DOI: 10.1016/S0377-0427 (00) 00632-4.

[Chi78] Theodore Seio Chihara. An Introduction to Orthogonal Polynomials. New York: Gordon e Breach, Science Publishers, 1978, p. 249. ISBN: 0-67704150-0.

[CI18] Ben Cox, Mee Seong Im. "On the module structure of the center of hyperelliptic Krichever-Novikov algebras". Em: Contemporary Mathematics 713 (2018). DOI: $10.1090 / \mathrm{conm} / 713$.

[CJ14] Ben Cox, Elizabeth Jurisich. "Realizations of the three-point Lie algebra $\operatorname{sl}(2, R) \oplus\left(\Omega_{R} / d R\right)^{\prime \prime}$. Em: Pacific Journal of Mathematics 270.1 (2014), pp. 27-48. DOI: 10.2140/pjm. 2014.270.27.

[Co189] Albert John Coleman. "The greatest mathematical paper of all time". Em: The Mathematical Intelligencer 11.3 (1989). ISSN: 03436993. DOI: 10. 1007/BF03025189.

[Cox+17] Ben Cox et al. "Simple superelliptic Lie algebras". Em: Communications in Contemporary Mathematics 19.3 (2017). DOI: 10.1142/S0219199716500322. 
[Cox08] Ben Cox. "Realizations of the four point affine Lie algebra $\operatorname{sl}(2, R) \oplus\left(\Omega_{R} / d R\right)$ ". Em: Pacific Journal of Mathematics 234.2 (2008), pp. 261-288. DOI: 10 . 2140/pjm. 2008.234.261.

[Cox16] Ben Cox. "On the universal central extension of hyperelliptic current algebras". Em: Proceedings of the American Mathematical Society 144 (2016), pp. 2825-2835. DOI: $10.1090 /$ proc/13057.

[Cox94] Ben Cox. "Verma modules induced from nonstandard Borel subalgebras". Em: Pacific Journal of Mathematics 165.2 (1994). ISSN: 0030-8730. DOI: $10.2140 / \mathrm{pjm} .1994 .165 .269$.

[Dat+83a] Etsuro Date et al. "Landau-Lifshitz equation: Solitons, quasi-periodic solutions and infinite-dimensional Lie algebras". Em: Journal of Physics A: Mathematical and General 16.2 (1983), pp. 221-236. ISSN: 03054470. DOI: 10.1088/0305-4470/16/2/006.

[Dat+83b] Etsuro Date et al. "Landau-\{L\}ifshitz equation: solutons, quasi-periodic solutions and infinite-dimensional \{L\}ie algebras". Em: Journal of Physics A: Mathematical and General 16.2 (1983), p. 221.

[Eti+11] Pavel Etingof et al. Introduction to representation theory. Vol. 59. Student Mathematical Library. American Mathematical Society, Providence, RI, 2011. ISBN: 978-0-8218-5351-1.

[FS93] Vyacheslav Futorny, Halip Saifi. "Modules of Verma type and new irreducible representations for affine Lie algebras". Em: Representations of algebras 14 (1993), pp. 185-191.

[FT01] Vyacheslav Futorny, Andrew Tsylke. "Classification of Irreducible Nonzero Level Modules with Finite-Dimensional Weight Spaces for Affine Lie Algebras". Em: Journal of Algebra 238.2 (2001). ISSN: 00218693. DOI: $10.1006 /$ jabr. 2000.8648 .

[Fut96] Vyacheslav Futorny. "Irreducible non-dense $A_{1}^{1}$-modules". Em: Pacific Journal of Mathematics 172.1 (1996). ISSN: 0030-8730. DOI: 10.2140/pjm. 1996.172.83.

[Fut97] Vyacheslav Futorny. “Representations of Affine Lie Algebras”. Em: Queen's papers in Pure and Applied Mathematics 106 (1997).

[Hum72] James Edward Humphreys. Introduction to Lie algebras and Representation Theory. Vol. 9. Graduate Texts in Mathematics. Springer New York, 1972. ISBN: 978-1-4612-6398-2.

[Ism05] Mourad E. H. Ismail. Classical and Quantum Orthogonal Polynomials in One Variable. Cambridge University Press, 2005. ISBN: 9780521782012. DOI: $10.1017 /$ CB09781107325982.

[JK89] Hans Plesner Jakobsen, Victor Gershevich Kac. "A new class of unitarizable highest weight representations of infinite dimensional Lie algebras". Em: Non-Linear Equations in Classical and Quantum Field Theory. Berlin, Heidelberg: Springer Berlin Heidelberg, 1989. DOI: 10 .1007/3540-15213-X_67.

[Kac94] Victor Gershevich Kac. Infinite-dimensional Lie algebras. Vol. 44. Progress in Mathematics. Cambridge university press, 1994. 
[Kas84] Christian Kassel. "Kähler differentials and coverings of complex simple Lie algebras extended over a commutative algebra". Em: Journal of Pure and Applied Algebra 34.2 (1984). Ed. por Proceedings of the Luminy conference on algebraic $\$ \mathrm{~K} \$$-theory, pp. 265-275. DOI: 10 . 1016/00224049 (84) 90040-9.

[KL82] Christian Kassel, Jean-Louis Loday. “Extensions centrales d'algèbres de Lie". Em: Annales de l'institut Fourier 32.4 (1982), pp. 119-142. DOI: 10. 5802/aif. 896.

[KL91] David Kazhdan, George Lusztig. "Affine Lie algebras and quantum groups". Em: International Mathematics Research Notices (1991). ISSN: 16870247. DOI: 10.1155/S1073792891000041.

[KL94] David Kazhdan, George Lusztig. "Tensor structures arising from affine Lie algebras. IV". Em: Journal of the American Mathematical Society (1994). ISSN: 1432-2218. DOI: $10.1007 / \mathrm{s} 00464-008-0019-5$.

[Kle07] Israel Kleiner. A history of abstract algebra. Boston: Birkhäuser Basel, 2007, p. 168. ISBN: 9780817646844. DOI: 10.1007/978-0-8176-4685-1.

[KN87] Igor Moiseevich Krichever, Sergei Petrovich Novikov. "Algebras of Virasoro type, Riemann surfaces and structures of the theory of solitons". Em: Functional Analysis and Its Applications 21.2 (1987), pp. 126-142. ISSN: 1573-8485. DOI: $10.1007 / \mathrm{BF} 01078026$.

[KN88] Igor Moiseevich Krichever, Sergei Petrovich Novikov. “Virasoro type algebras, Riemann surfaces and strings in Minkowski space". Em: Funkts. Anal. Prilozhen. 21.4 (1988), pp. 47-61. DOI: 10.1007/BF01077803.

[KR87] Victor Gershevich Kac, Ashok Raina. Bombay lectures on highest weight representations of infinite dimensional Lie algebras. Vol. 2. World Scientific Publishing, 1987. DOI: $10.1142 / 8882$.

[MF18] Daniel Cordeiro de Morais Filho. Manual de Redação Matemática. Ed. por Hilário Alencar. $2^{\mathrm{a}}$ ed. Rio de Janeiro: Sociedade Brasileira de Matemática, 2018.

[MP95] Robert Vaughan Moody, Arturo Pianzola. Lie algebras with triangular decompositions. Wiley-Interscience Publication, 1995, p. 712. ISBN: 978-0471-63304-4.

[MS19] Andreas Malmendier, Tony Shaska. "From hyperelliptic to superelliptic curves". Em: Albanian Journal of Mathematics 13.1 (2019), 107-200.

[Pol50] Félix Pollaczek. "Sur une famille de polynomes orthogonaux a 4 parametres". Em: Comptes rendus hebdomadaires des seances de l'academie des sciences 230.26 (1950), pp. 2254-2256.

[San p] Felipe Albino dos Santos. "On the universal central extension of superelliptic affine Lie algebras". Em: Communications in Algebra (no prelo para 2021).

[San17a] Felipe Albino dos Santos. "Irreducible $\varphi$-Verma modules for hyperelliptic Heisenberg algebras". Em: arXiv:1709.05663 [math.RT] (2017). URL: http://arxiv.org/abs/1709.05663.

[San17b] Felipe Albino dos Santos. "Módulos irredutíveis para subálgebras de Heisenberg de álgebras de Krichever-Novikov". Dissertação de mestrado. Universidade de São Paulo, 2017. DOI: 10 . 11606/ D . 45 . 2017 . tde-05122017-200848. 
[Sch03] Martin Schlichenmaier. "Local cocycles and central extensions for multipoint algebras of Krichever-Novikov type". Em: Journal für die reine und angewandte Mathematik (Crelles Journal) 559 (2003), pp. 53-94. ISSN: 0075-4102. DOI: 10.1515/crll.2003.052.

[Sch07] Martin Schlichenmaier. An Introduction to Riemann Surfaces, Algebraic Curves and Moduli Spaces. Springer-Verlag Berlin Heidelberg, 2007, p. 217. ISBN: 978-3-540-71174-2. DOI: $10.1007 / \mathrm{bfb0113492.}$

[Sch14a] Martin Schlichenmaier. "From the Virasoro algebra to Krichever-Novikov Type Algebras and Beyond". Em: Harmonic and complex analysis and its applications. Springer, 2014, pp. 325-358.

[Sch14b] Martin Schlichenmaier. Krichever-Novikov Type Algebras. Luxembourg: Walter de Gruyter GmbH, 2014, p. 360. ISBN: 978-3-11-027964-1. DOI: 10.1515/9783110279641.

[Sch90a] Martin Schlichenmaier. "Central extensions and semi-infinite wedge representations of Krichever-Novikov algebras for more than two points". Em: Letters in Mathematical Physics (1990). ISSN: 03779017. DOI: 10.1007/ BF00417227.

[Sch90b] Martin Schlichenmaier. "Krichever-Novikov algebras for more than two points: Explicit generators". Em: Letters in Mathematical Physics 19.4 (1990), pp. 327-336. ISSN: 03779017. DOI: 10.1007/BF00429952.

[Ste+76] Norman Earl Steenrod et al. How to Write Mathematics. American Mathematical Society, 1976. ISBN: 978-0-8218-0055-3.

[Sze97] Gabor Szegö. Orthogonal Polynomials. Vol. XXIII. Colloquium publications. American Mathematical Society, 1997, pp. 1-56. ISBN: 9781479923748. DOI: $10.1016 / \mathrm{S} 0377-0427$ (98)00171-X. 


\section{Índice}

álgebra afim

3-ponto, 27

4-ponto, 26

DJKM, 29, 30

elíptica, 28

hiperelíptica, 28

N-ponto, 26

superelíptica, 31

álgebra corrente, 19

álgebra corrente de gênero maior, 20

álgebra de Heisenberg, 6

álgebra de Heisenberg hiperelíptica, 69

álgebra de Kac-Moody, 13

álgebra de Kac-Moody afim não torcida, 16

álgebra de Krichever-Novikov, 21

álgebra de Lie, 6

abeliana, 7

afim, 13

graduada, 9

hiperelíptica, 68

perfeita, 8,20

redutível, 8

semisimples, 8

simples, 8

soma direta, 8

álgebra de Lie especial linear, 6

álgebra de Virasoro, 21

álgebra de funções, 19

álgebra dos endomorfismos, 6

álgebra envelopante universal, 8

álgebra fortemente quasegraduada,

22

álgebra fracamente quasegraduada,

22

álgebra laço

3-ponto, 27

4-ponto, 26

DJKM, 29

elíptica, 28

hiperelíptica, 28 álgebra tensorial, 8

álgebras superelípticas, 31

álgebras superelípticas afim, 32

ação de Lie, 10

Adrien-Marie Legendre, 39

algebra $N$-ponto, 20

algebra multiponto, 20

anel

N-ponto, 25

3-ponto, 27

4-ponto, 25

DJKM, 29

elíptico, 28

hiperelíptico, 28

aplicação simétrica bilinear, 11

base de coraízes, 12

base de raízes, 12

centro, 7

cisão de pontuações, 21

cociclo, 24

cohomologia de complexo, 24

colchete de Lie, 6

complexo, 23

complexo de de Rham, 23

comutador, 6

coraízes simples, 12

curva algébrica, 18

curva algébrica plana afim, 18

curva algébrica projetiva, 18

curva elíptica, 18

curva hiperelíptica, 18, 28

curvas superelípticas, 31

decomposição em espaços de raízes, 12,65

decomposição triangular, 13, 65

delta de Kronecker, 7

diagramas de Dynkin, 13

diferenciais, 23

diferenciais de Kähler, 25 
diferenciais de Kähler módulo formas exatas, 32

diferenciais exatos, 25

elemento central canônico, 66

elementos homogêneos, 10, 11, 22

esfera de Riemann, 18

espaço da representação, 10

espaço de raízes imaginárias, 66

espaço vetorial graduado, 9

extensão, 24

extensão central, 24

extensão central universal, 24

extensões centrais equivalentes, 24

forma de Cartan-Killing, 11

função Gamma, 40

função hipergeométrica, 42

função holomorfa, 19

função meromorfa, 19

função peso, 40

Gábor Szegő, 39

geradores Chevalley, 13

graduação trivial, 10

homomorfismo de Lie, 7

ideal de Lie, 7

identidade de Jacobi, 6

invariante, 11

módulo

$\varphi$-Verma, 66

de diferenciais, 33

de diferenciais de Kähler, 25

de Lie, 10

de peso máximo, 64

de Verma, 64

do tipo Verma, 65

graduado, 11

indecomponível, 10

irredutível, 10

quasegraduado, 22

matriz de adjacência, 13

matriz de Cartan, 13

matriz de Cartan afim, 13

matriz de Cartan generalizada, 11

matriz positiva definida, 13 nível, 65

par de peso máximo, 64

peso (de uma raiz), 12

peso máximo, 64

polinômios

associados, 42

de Chebyshev, 41

de Gegenbauer, 40

de Jacobi, 40

de Jacobi associados, 42

de Laguerre, 41

de Laurent, 19

de Pollaczek, 41

ortogonais, 40

ortogonais clássicos, 42

ultra-esféricos, 40

ultraesféricos associados, 59

polo, 19

pontos marcados, 21

pontuações, 21

posto, 13

quasegraduação, 22

quasegraduação de álgebra de Lie, 22

quasi-graduation, 22

raízes simples, 12

raiz imaginária indivisível, 66

realização, 12

representação adjunta, 10

representação de Lie, 10

símbolo de Pochhammer, 40

sequência exata curta, 24

sistema de raízes, 65

situação clássica, 22

subálgebra de Cartan, 13

subálgebra de Lie, 7

subálgebra derivada, 7

subespaço homogêneo, 10, 11, 22

submódulo gerado, 64

superfície de Riemann, 18

toro, 18

vetor de peso, 64

vetor de peso máximo, 64 